Publ. RIMS, Kyoto Univ.

15 (1979), 53-157

\title{
Boundary Layers and Homogenization of Transport Processes
}

\author{
By \\ Alain Bensoussan*. Jacques L. LiONS* \\ and George C. PapanicolaOU**1)
}

\section{Contenits}

$\S 0$. Introduction.

$\S 1$. Physical theory of transport processes.

1. 1. The transport equation.

1. 2. Boundary conditions. $\quad 57$

1. 3. Existence and uniqueness. 60

1. 4. Cellular geometry, homogenization and force fields. 64

1. 5. Half-space problems. 66

$\S 2$. Probabilistic theory of transport processes.

2.1. Construction of transport processes. 71

2. 2. Boundary conditions. $\quad 75$

2. 3. Connection with the physical theory. 83

2. 4. Asymptotic problems and homogenization. 87

2.5. Ergodic properties of transport operators. 89

2.6. Reflection and transmission operators for $\begin{array}{lr}\text { half-space problems. } & 96\end{array}$

2. 7. Potential theory for the half-space problem. 106

$\S 3$. Diffusion approximations.

3. 1. Asymptotic expansion in an unbounded region and homogenization.

Communicated by K. Itô, July 7, 1976. Revised August 15, 1977.

* I. R. I. A., Laboria, Laboratoire de Recherche en Informatique et Automatique, Domaine de Voluceau-Rocquencourt, 78150 Le Chesnay, France.

** Courant Institute of Mathematical Sciences, New York University, 251 Mercer Street, New York, N. Y. 10012, U. S. A.

1) Supported by an Alfred P. Sloan Foundation Fellowship and by the Air Force Office of Scientific Research under Grant No. AFOSR-76-2884. 
3. 2. Validity of the expansion.

3. 3. Weak convergence of the process.

3. 4. Boundary layer coordinates.

3. 5. Asymptotic expansions for absorbing boundary conditions (without cells).

3.6. Asymptotic expansion for reflecting boundary conditions (without cells).

3. 7. Weak convergence of reflected process. 146 References

\section{§. Introduction}

This work started as a continuation of [1] but in the meantime the scope of the project widened and the intimate connection with homogenization problems $[2,3]$ became apparent. The contents are briefly as follows.

In Section 1 we review for completeness the physics of transport processes. We focus on those points that bear upon the asymptotic analysis when the mean free path tends to zero. We refer to [1] for many references to related work and to $[4,5]$ for additional information on asymptotic problems.

In Section 2 we give a probabilistic description of linear transport processes much like in [1]. The material is standard in the theory of Markov processes. The theorem at the end of Section 2.5 and the results of Sections 2.6 and 2.7 are of direct interest to the asymptotics. They are also of independent interest.

Section 3 contains the main results, namely the asymptotic limit of small mean free path in transport theory. Without cellular-space structure (i.e., without homogenization) the results are fairly complete although interface problems are not treated. With cellular structure and boundary layers the analysis has yet to be carried out. We employ the theory of Stroock and Varadhan [6] which seems to be just the right tool for our problems.

The analysis herein is restricted to transport problems that involve scattering only (no fission) to highest order in the mean free path parameter. Processes that involve particle creation, multiplicative processes, can 
be formulated as branching transport processes. Their asymptotic analysis requires several additional considerations not given here (cf. [21]).

The general scheme by which asymptotic results are obtained is to first construct formal asymptotic expansions in the usual way as in $[4,5$, 8] and in Sections 3.1, 3. 5 and 3.6. Sections 2. 5, 2.6 and 2. 7 provide simple sufficient conditions for the existence of such expansions. Then we prove that the expansions are truly asymptotic. To prove limit theorems (invariance principles) we follow along the lines of [6] and the set-up abstracted by Kurtz in [7]; this is done in 3.3 and 3.7.

We thank M. Williams and E. Larsen for many discussions on the problems considered here. We also thank the referee of the paper for carefully reading the manuscript and suggesting many improvements and corrections.

Finally we thank S. R. S. Varadhan for generously sharing with us his insight into the problems considered here and introducing us to many important ideas and techniques which enter into much of what follows.

\section{$\S \mathbb{1}$. Physical Theory of Transport Processes}

\section{1. The Transpont Equation}

In many physical phenomena the quantities of interest satisfy, within certain reasonable approximations, linear transport equations. Radiative transport theory $[9,10]$ and neutron transport theory $[11,12,13]$ are perhaps the best known examples of physical theories leading to the transport equation we shall study here. Gas dynamics on the other hand leads to Boltzmann's equation $[14,15,16]$ which is nonlinear. The relevant linearizations of this equation do not admit the kind of probabilistic treatment we intend to give so we shall not discuss gas dynamics here.

Let $\phi(t, x, v)$ denote the density of particles at time $t \geq 0$, at location $x \in \mathbb{R}^{3}$ and with velocity $v \in \mathbb{R}^{3}$. The word "particles" stands for photons or neutrons depending on the context and will be used throughout in a generic sense. The particles move on straight lines in the absence of collisions. We assume that they collide with obstacles in the underlying medium and that the latter are not affected by the collisions; the particles 
do not collide with each other. The last assumption leads to a linear conservation equation for $\phi$ which we now describe.

In the interval $(t, t+\lrcorner t)$ we have

$$
\begin{aligned}
\phi(t+ & \Delta t, x+v \Delta t, v)-\phi(t, x, v) \\
& =\left(\frac{\partial \phi(t, x, v)}{\partial t}+v \cdot \frac{\partial \phi(t, x, v)}{\partial x}\right) \Delta t+o(\Delta t) .
\end{aligned}
$$

This is the total derivative of $\phi$ and $v \cdot \frac{\partial}{\partial x}$ stands for the dot product of $v$ and the $x$ gradient. In the same time interval $\phi(t, x, v)$ increases as a result of collisions at $x, t$ which convert particles of velocity $v^{\prime} \neq v$ into particles of velocity $v$. It decreases as a result of collisions that convert particles of velocity $v$ to velocities $v^{\prime} \neq v$. Let $\Sigma\left(t, x, v, v^{\prime}\right)$ clenote the fraction of particles per unit time converted from velocity $v^{\prime}$ to velocity $v$ and assume it is a continuous function of $v$ and $v^{\prime}$. Let $\sigma(t, x, v)$ denote the fraction of particles per unit time converted to velocity $v^{\prime} \neq v$. Then we have

$$
\begin{aligned}
\phi(t & +\Delta t, x+v \Delta t, v)-\phi(t, x, v) \\
& =\left(\int \Sigma\left(t, x, v, v^{\prime}\right) \phi\left(t, x, v^{\prime}\right) d v^{\prime}-\sigma(t, x, v) \phi(t, x, v)\right) \Delta t+o(\Delta t)
\end{aligned}
$$

where the integral is over all velocities in $\boldsymbol{R}^{3}$. Combining the above expressions we get the transport equation

$$
\begin{aligned}
& \frac{\partial \phi(t, x, v)}{\partial t}+v \cdot \frac{\partial \phi(t, x, v)}{\partial x} \\
& \quad=\int \Sigma\left(t, x, v, v^{\prime}\right) \phi\left(t, x, v^{\prime}\right) d v^{\prime}-\sigma(t, x, v) \phi(t, x, v) .
\end{aligned}
$$

The functions $\Sigma$ and $\sigma$ are called the differential and the total scattering cross-section respectively.

Equation (1.1.1) must be supplemented with initial and boundary conditions. In the absence of boundaries, $x \in \boldsymbol{R}^{3}$ and (1.1.1) is to hold in all of $\boldsymbol{R}^{3}$ for both $x$ and $v$ and

$$
\phi(0, x, v)=\phi_{0}(x, v)
$$

a given initial particle density. Boundary conditions are considered in the next section. 
If for each $x$ and $t$ we have

$$
\int \Sigma\left(t, x, v^{\prime}, v\right) d v^{\prime}=\sigma(t, x, v)
$$

then the collisions are called pointwise conservative; there is no creation or anihilation of particles. As a consequence $\iint \phi d v d x$, the total number of particles, does not change in time. If the left hand side of (1.1.3) exceeds the right hand side then we have net creation of particles; otherwise net destruction. In many important problems (1.1.3) does not hold pointwise but the solutions of (1.1.1) admit nontrivial steady-state behavior. Such situations are also called conservative but they are clearly different from (1.1.3). To emphasize the difference we shall refer to (1.1.3) as the pointwise conservative case.

\section{2 Boundary Conditions}

Let $D$ be a domain in $\boldsymbol{R}^{3}$. A typical boundary value problem is the following

$$
\begin{aligned}
& \text { (1.2.1) } \frac{\partial \phi}{\partial t}+v \cdot \frac{\partial \phi}{\partial x}=\int \Sigma \phi d v^{\prime}-\sigma \phi, \quad x \in \mathscr{D}, v \in \boldsymbol{R}^{3}, t>0 \text {, } \\
& \phi(0, x, v)=\phi_{0}(x, v), \quad x \in \mathscr{D}, v \in \mathbb{R}^{3}, \\
& -\hat{n} \cdot v \phi(t, x, v)=g(t, x, v), \quad t>0, x \in \partial \mathscr{D}, \hat{n} \cdot v<0 .
\end{aligned}
$$

Here $\hat{n}$ denotes the unit outer normal to the boundary $\partial \mathscr{D}$ of $\mathscr{D}$. Problem (1.2.1) is well defined as can be seen from the following considerations. Let $d S$ denote a surface element at $x$ with normal $\hat{n}$. Then $\hat{n} \cdot v \phi(t, x, v) d S d v d t$ is the number of particles crossing $d S$ at $x$ with velocity $v$ within $d v$ and at time $t$ within $d t$. To confirm this we integrate (1.2.1) and use Green's Theorem to obtain the identity

$$
\text { (1.2.2) } \begin{aligned}
\int_{\mathscr{D}} \int \phi(t, x, v) d v d x+ & \int_{0}^{t} \int_{\partial \mathscr{D}} \int_{\hat{n} \cdot v>0} \hat{n} \cdot v \phi(s, x, v) d v d S d s \\
=\int_{\mathscr{D}} \int_{0} \phi_{0}(x, v) d x d v & \int_{\|}^{t} \int_{\mathscr{I}} \int_{s} \sigma_{s}(s, x, v) \phi(t, x, v) d v d x d s \\
& +\int_{0}^{t} \int_{\partial \mathscr{D}} \int_{\hat{\imath} \cdot v<0} g(s, x, v) d v d S d s
\end{aligned}
$$


The left hand side of (1.2.2) represents the total number of particles in $\mathscr{D}$ at time $t$ plus the total number of particles that exited from $\mathscr{D}$ up to time $t$. The right hand side is the total number of particles present at time $t=0$ plus the total number that entered $\mathscr{D}$ through the boundary up to time $t$ and (the middle term) the total number that were created in $\mathscr{D}$ up to time $t$. We have defined $\sigma_{s}$ by

$$
\sigma_{s}(t, x, v)=\int \Sigma\left(t, x, v^{\prime}, v\right) d v^{\prime}-\sigma(t, x, v)
$$

Uniqueness for (1.2.1) is easily established from (1.2.2), assuming that $\left|\sigma_{s}\right|<M<\infty$, because $\phi \geq 0$ and we have the inequality

$\Phi(t) \leq \int_{\mathscr{D}} \int_{0} \phi_{0}(x, v) d x d v+\int_{0}^{t} \int_{\partial \mathscr{D}} \int_{\hat{n} \cdot v<0} g(s, x, v) d v d S d s+M \int_{0}^{\iota} \Phi(s) d s$, where

$$
\Phi(t)=\int_{\mathscr{D}} \int \phi(t, x, v) d v d x
$$

Existence is discussed in the next section and again in Section 2.1 in a probabilistic setting.

Another typical boundary value problem involves reflection of particles at the boundary. Let $B\left(t, x, v, v^{\prime}\right)$ denote the fraction of particles which at the point $x \in \partial \mathscr{D}$ and at time $t$ are converted from outgoing with velocity $v^{\prime}\left(\hat{n} \cdot v^{\prime}>0\right)$ to incoming with velocity $v(\hat{n} \cdot v<0)$. The reflecting boundary value problem is then

(1.2.4) $\frac{\partial \phi}{\partial t}+v \cdot \frac{\partial \phi}{\partial x}=\int \Sigma \phi d v^{\prime}-\sigma \phi, \quad x \in \mathscr{D}, v \in \boldsymbol{R}^{3}, t>0$,

$$
\phi(0, x, v)=\phi_{0}(x, v), \quad x \in \mathscr{D}, v \in \boldsymbol{R}^{3}
$$

(1.2.5) $-\hat{n} \cdot v \phi(t, x, v)$

$$
\begin{gathered}
=\int_{\hat{n} \cdot v^{\prime}>0} B\left(t, x, v, v^{\prime}\right) \hat{n} \cdot v^{\prime} \phi\left(t, x, v^{\prime}\right) d v^{\prime}+g(t, x, v), \\
t>0, x \in \partial \mathscr{D}, \hat{n} \cdot v<0 .
\end{gathered}
$$

The left hand side of (1.2.5) is the flux of particles incoming at the boundary and the right hand side is the incoming flux due to reflection plus the incoming flux due to exterior sources. 
Let us denote by $\sigma_{B}(t, x, v), \hat{n} \cdot v>0$, the fraction of particles reflected at $x \in \partial \mathscr{D}, t>0$, which arrived at the boundary with velocity $v$. Then,

$$
\sigma_{B}(t, x, v)=\int_{\hat{n} \cdot v^{\prime}<0} B\left(t, x, v^{\prime}, v\right) d v^{\prime}
$$

and hence, from (1.2.5)

$$
\begin{aligned}
&(1.2 .7)--\int_{\hat{n} \cdot v<0} \hat{n} \cdot v \phi(t, x, v) d v \\
&= \int_{\hat{n} \cdot v>0} \sigma_{B}(t, x, v) \hat{n} \cdot v \phi(t, x, v) d v+\int_{\hat{n} \cdot v<0} g(t, x, v) d v, \\
& t>0, x \in \partial \mathscr{D} .
\end{aligned}
$$

Equation (1.2.7) is the analog of (1.2.2) at the boundary points, i.e., it is the law of conservation of flux at the boundary.

When $\sigma_{B}(t, x, v) \equiv 1$ then we have total reflection at the boundary. In general, $0 \leq \sigma_{B}(t, x, v) \leq 1$ and strict inequality on the right corresponds to partial reflection at the boundary.

At the interface between two adjoining media boundary conditions are imposed to preserve continuity of flux. Thus, if $\phi_{1}(t, x, v)$ and $\phi_{2}(t, x, v)$ denote the particle density in $\mathscr{D}_{1}$ and $\mathscr{D}_{2}$ respectively, we have the following boundary value problem (assume no reflection).

$$
\begin{gathered}
\frac{\partial \phi_{1}}{\partial t}+v \cdot \frac{\partial \phi_{1}}{\partial x}=\int \Sigma_{1} \phi_{1} d v^{\prime}-\sigma_{1} \phi_{1}, \quad x \in \mathscr{D}_{1}, v \in \boldsymbol{R}^{3}, t>0 \\
\frac{\partial \phi_{2}}{\partial t}+v \cdot \frac{\partial \phi_{2}}{\partial x}=\int \Sigma_{2} \phi_{2} d v^{\prime}-\sigma_{2} \phi_{2}, \quad x \in \mathscr{D}_{2}, v \in \mathbb{R}^{3}, t>0, \\
\phi_{1}(0, x, v)=\phi_{01}(x, v) \quad x \in \mathscr{D}_{1} \\
\phi_{2}(0, x, v)=\phi_{02}(x, v) \quad x \in \mathscr{D}_{2} \\
-\hat{n}_{1} \cdot v \phi_{1}(t, x, v)=g_{1}(t, x, v), \\
x \in \partial \mathscr{D}_{1}, x \notin \partial \mathscr{D}_{1} \cap \partial \mathscr{D}_{2}, \hat{n}_{1} \cdot v<0 \\
-\hat{n}_{2} \cdot v \phi_{2}(t, x, v)=g_{2}(t, x, v), \\
x \in \partial \mathscr{D}_{1}, x \neq \partial \mathscr{D}_{1} \cap \partial \mathscr{I}_{2}, \hat{n}_{2} \cdot v<0 \\
-\hat{n}_{1} \cdot v \phi_{1}(t, x, v)=\hat{n}_{2} \cdot v \phi_{2}(t, x, v),
\end{gathered}
$$




$$
\begin{aligned}
\hat{n}_{1} \cdot v<0, \quad \hat{n}_{2} \cdot v>0, \\
-\hat{n}_{2} \cdot v \phi_{2}(t, x, v)=\hat{n}_{1} \cdot v \phi_{1}(t, x, v), \\
\hat{n}_{2} \cdot v<0, \quad \hat{n}_{1} \cdot v>0, x \in \partial \mathscr{D}_{1} \cap \partial \mathscr{D}_{2} .
\end{aligned}
$$

Clearly (1.2.11) can be written simply as (since $\hat{n}_{1}=-\hat{n}_{2}$ )

$$
\text { (1. 2. } \left.11^{\prime}\right) \quad \phi_{1}(t, x, v)=\phi_{2}(t, x, v), \quad x \in \partial \mathscr{D}_{1} \cap \partial \mathscr{D}_{2}, v \in \boldsymbol{R}^{3} .
$$

However, it is appropriate to vrite (1.2.11) since this is the general statement of flux continuity. If the motion inside $\mathscr{D}_{1}$ and $\mathscr{D}_{2}$ is not linear between collisions (see the probabilistic treatment), then the multiplicative factors in (1.2.11) need not cancel in general.

\section{3. Existence and Uniqueness}

The existence of solutions to (1.2.1) which we shall outline yields also that $\phi$ is nonnegative if the data is nonnegative. Therefore, the conservation law (1.2.2) yields uniqueness if $\left|\sigma_{s}\right|<M<\infty$. In the partially reflecting case instead of (1.2.2) we have the conservation equation

$$
\begin{gathered}
\int_{\mathscr{Q}} \int \phi(t, x, v) d v d x+\int_{0}^{t} \int_{\partial \mathscr{D}} \int_{\hat{n} \cdot v>0} \hat{n} \cdot v \phi(s, x, v) d v d S d s \\
=\int_{\mathscr{D}} \int \phi_{0}(x, v) d x d v+\int_{0}^{t} \int_{\mathscr{D}} \int_{s} \sigma_{s}(s, x, v) \phi(s, x, v) d v d x d s \\
+\int_{0}^{t} \int_{\partial \mathscr{D}} \int_{\hat{n} \cdot v>0} \sigma_{B}(s, x, v) \hat{n} \cdot v \phi(s, x, v) d v d S d s \\
+\int_{0}^{t} \int_{\partial \mathscr{D}} \int_{\hat{n} \cdot v>0} g(s, x, v) d v d S d s .
\end{gathered}
$$

Here we have used (1.2.6). From (1.3.1) and the fact that $0 \leq \sigma_{B} \leq 1$ uniqueness follows as for (1.2.1).

We pass now to the question of existence and we shall restrict attention to (1.2.1) with $\mathscr{D}$ a bounded open set in $\boldsymbol{R}^{3}$. One must distinguish two cases here. When the differential scattering cross-section $\Sigma\left(t, x, v, v^{\prime}\right)$ is a symmetric function of $v$ and $v^{\prime}$ the existence theory for (1.2.1) is identical to the one for its adjoint and thus it will follow from the general considerations of Sections 2.1, 2.2. Problem (1.2.1) is wellposed under very general conditions on the data and for $t \in[0, T], T<\infty$ 
but arbitrary.

When $\Sigma$ is not symmetric the existence theory for (1.2.1) follows in a weak sense from the probabilistic treatment. The iteration argument below leads directly to the desired results as we now show.

First we rewrite (1.2.1) as an integral equation by the usual method of characteristics. Let $t=t_{\mathscr{D}}(x, v)$ denote the time for a particle to reach $\partial \mathscr{D}$ starting from $x \in \mathscr{D}$ and moving with velocity $-v$. Then we have

$$
\begin{aligned}
\phi(t, x, v) & =\chi\left(\iota<t_{\mathscr{D}}\right) \phi_{0}(x-v t, v) \\
& \times \exp \left(-\int_{0}^{t} \sigma(s, x-v(t-s), v) d s\right) \\
+ & \%\left(t \geq t_{\mathscr{D}}\right) \frac{-1}{\hat{n} \cdot v} g\left(t-t_{\mathscr{D}}, x-v t_{\mathscr{D}}, v\right) \\
\times & \exp \left(-\int_{t-t_{\mathscr{D}}}^{t} \sigma(s, x-v(t-s), v) d s\right) \\
+ & \int_{t-t \wedge t_{\mathscr{D}}}^{t} \exp \left(-\int_{s}^{t} \sigma(\gamma, x-v(t-\gamma), v) d \gamma\right) \\
& \times \int \Sigma\left(s, x-v(l-s), v, v^{\prime}\right) \phi\left(s, x-v(t-s), v^{\prime}\right) d v^{\prime} d s .
\end{aligned}
$$

Here $t \wedge t_{\mathscr{D}}=\min \left(\iota, t_{\mathscr{\Omega}}\right)$ and $\chi\left(t \leq t_{\mathscr{D}}\right)$ equals one if $t \leq t_{\mathscr{D}}$ and zero otherwise. We assume that the data $\phi_{0}$ and $g$ are compatible, i.e. for $x \in \partial \mathscr{D}$, $\hat{n} \cdot v<0, \phi_{0}(x, v)=g(0, x, v)$. We also assume that $-(\hat{n} \cdot v)^{-1} g(t, x, v)$ is uniformly bounded $x \in \partial \mathscr{D}, \hat{n} \cdot v \leq 0$ and $0 \leq t \leq T<\infty$.

Regarding the $v$-behavior of the data $\phi_{0} \geq 0$ and $g \geq 0$, and the scattering cross-section $\Sigma$ we assume the following

$$
\int \sup _{x \in ! D} \phi_{0}(x, v) d v<\infty \text {, }
$$

$$
\begin{aligned}
& \int \sup _{t \geq 0} \sup _{x \in \Omega \mathscr{D}} g(t, x, v) d v<\infty, \\
& \int \sup _{t \geq 0} \sup _{x \in \bar{D}} \sup _{v \in \boldsymbol{R}^{s}} \sum\left(t, x, v^{\prime}, v\right) d v^{\prime}<\infty .
\end{aligned}
$$

These hypotheses are quite reasonable especially when there is a cut-off in velocity space so only velocities of up to a finite magnitude enter into the problem. Regarding the total scattering cross-section $\sigma$ we assume that 


$$
0 \leq \sigma \leq M \leq \infty
$$

Now (1.3.2) can be solved by iteration. We let

(1.3.7) $\phi^{(0)}(t, x, v)$

$$
\begin{aligned}
= & \chi\left(t<t_{\mathscr{D}}\right) \phi_{0}(x-v t, v) \exp \left(-\int_{0}^{t} \sigma(s, x-v(t-s), v) d s\right) \\
& +\chi\left(t \geq t_{\mathscr{D}}\right) \frac{-1}{\hat{n} \cdot v} g\left(t-t_{\mathscr{D}}, x-v t_{\mathscr{D}}, v\right) \\
& \times \exp \left(-\int_{t-t_{\mathscr{D}}}^{t} \sigma(s, x-v(t-s), v) d s\right)
\end{aligned}
$$

and

$$
\phi^{(n-1)}(t, x, v)=K_{t} \phi^{(n)}(t, x, v) \quad n=1,2, \cdots
$$

where

$$
\begin{aligned}
\text { (1. 3.9) } \quad K_{t} \phi(t, x, v) & =\int_{t-t \wedge t \mathscr{D}}^{t} \exp \left(-\int_{s}^{t} \sigma(\gamma, x-v(t-\gamma), v) d \gamma\right) \\
& \times \int \Sigma\left(s, x-v(t-s), v, v^{\prime}\right) \phi\left(s, x-v(t-s), v^{\prime}\right) d v^{\prime} d s .
\end{aligned}
$$

From these definitions we find that

$$
\int \sup _{x \in \overline{\mathscr{D}}} \sup _{t \geq 0} \phi^{(0)}(t, x, v) d v \leq C<\infty
$$

and inductively

$$
\int \sup _{x \in \bar{D}} \phi^{(n)}(t, x, v) d v \leq \frac{\left(C^{\prime} t\right)^{n}}{n !} C
$$

where $C$ and $C^{\prime}$ are constants in view of our assumptions. Since the solution $\phi$ of (1.3.2) is formally

$$
\phi=\sum_{n=0}^{\infty} \phi^{(n)}
$$

our estimates above show that this series converges and $\phi$ exists in the norm

$$
\int \sup _{0 \leq s \leq t} \sup _{x \in \mathscr{D}}|\phi(s, x, v)| d v \quad(t<\infty) .
$$

This concludes the proof of existence. 
The bounds obtained above for the iteration process are, of course, very crude and behave very poorly for $t$ large. On the other hand the large $t$ behavior of solutions to (1.3.2) is a much more difficult problem; it is actually an eigenvalue problem. Uniqueness, as observed earlier, follows simply from conservation relations such as (1.2.2) or also, from (1. 3. 2).

The reflecting boundary value problem (1.2.4)-(1.2.5) is treated as follows. We rewrite the equation and boundary conditions in integral form.

$$
\begin{aligned}
& \phi(t, x, v)=\chi\left(t<t_{\mathscr{D}}\right) \phi_{0}(x-v \iota, v) \\
& \quad \times \exp \left(-\int_{0}^{t} \sigma(s, x-v(t-s), v) d s\right)+\chi\left(t \geq t_{\mathscr{D}}\right) \\
& \quad \times\left[\frac { - 1 } { \hat { n } \cdot v } \int _ { \hat { n } \cdot v ^ { \prime } > 0 } B ( t - t _ { \mathfrak { D } } , x - v t _ { \mathscr { D } } , v , v ^ { \prime } ) \hat { n } \cdot v ^ { \prime } \phi \left(t-t_{\mathscr{D}},\right.\right. \\
& \left.\left.x-v t_{\mathscr{D}}, v^{\prime}\right) d v^{\prime} \frac{-1}{\hat{n} \cdot v} g\left(t-t_{\mathscr{D}}, x-v t_{\mathfrak{D}}, v\right)\right] \\
& \quad \times \exp \left(-\int_{t-t_{\mathscr{D}}}^{t} \sigma(s, x-v(t-s), v) d s\right) \\
& \quad+\int_{t-\imath \wedge t_{\mathscr{D}}}^{t} \exp \left(-\int_{s}^{t} \sigma(\gamma, x-v(\iota-\gamma), v) d \gamma\right) \\
& \quad \times \int \Sigma\left(s, x-v(t-s), v, v^{\prime}\right) \phi\left(s, x-v(t-s), v^{\prime}\right) d v^{\prime} d s .
\end{aligned}
$$

It is clear that even if the velocities are effectively cut off from above and below (in magnitude), there still are singularities in (1.3.11) due to grazing angles at the boundary. In the probabilistic context one can give reasonably sharp conditions for existence (in a natural way). This is done in Sections 2.1 and 2.2 ahead. A crude sufficient condition for the usual iteration scheme associated with (1.3.11) to converge is the following

$$
\text { (1. 3. 12) } \quad \int \frac{-1}{\hat{n} \cdot v}\left[\sup _{t \geq 0} \sup _{x \in \partial \mathscr{D}} \sup _{v^{\prime}} B\left(t, x, v, v^{\prime}\right) \hat{n} \cdot v^{\prime}\right] d v<1 \text {. }
$$

The physical meaning of (1.3.12) is that the reflection process is suffciently weak, in a uniform sense, and near grazing angles emerge from reflection with very small probabilities. 


\section{4. Cellular Geometry, Homogenization, Force Fields}

Exact solutions for any of the boundary value problems of Section 1.2 are rarely available except for simple geometries and, essentially, homogeneous media. On the other hand direct numerical solution is also out of the question because the problems are too big and badly conditioned. Therefore, it is necessary to treat these problems analytically at first before doing any computation. Fortunately a small parameter is usually available so asymptotic analysis is called for.

The small parameter is the mean free path between collisions measured in units with respect to which the size of the region of interest $\mathscr{D}$ is of order 1 . We shall denote this parameter by $\varepsilon>0$. In many situations, such as in the case of nuclear reactors, the scattering cross-sections $\Sigma\left(x, v, v^{\prime}\right)$ and $\sigma(x, v)$ (assumed time independent) vary with $x$ in an approximately periodic manner and with spatial period comparable to the mean free path $\varepsilon$, i.e. small. If we let $y=\frac{x}{\varepsilon}$ be the spatial variable measured in units where the mean free path is one then $\Sigma$ and $\sigma$ are actually functions of $x, y, v$ and $v^{\prime}$ i.e.,

$$
\Sigma=\Sigma\left(x, \frac{x}{\varepsilon}, v, v^{\prime}\right), \quad \sigma=\sigma\left(x, \frac{x}{\varepsilon}, v\right)
$$

and for each fixed $x, v, v^{\prime}, \Sigma\left(x, y, v, v^{\prime}\right)$ and $\sigma(x, y, v)$ are periodic functions of $y$ with period 1 in all coordinate directions.

Problem (1.2.1) in scaled form becomes now

$$
\begin{aligned}
& \frac{\partial \phi^{\varepsilon}(t, x, v)}{\partial t}+\frac{1}{\varepsilon} v \cdot \frac{\partial \phi^{\varepsilon}(t, x, v)}{\partial x} \\
& =\frac{1}{\varepsilon^{2}} \int \Sigma\left(x, \frac{x}{\varepsilon}, v, v^{\prime}\right) \phi^{\varepsilon}\left(t, x, v^{\prime}\right) d v^{\prime} \\
& \quad-\frac{1}{\varepsilon^{2}} \sigma\left(x, \frac{x}{\varepsilon}, v\right) \phi^{\varepsilon}(t, x, v), \quad x \in \mathscr{D}, v \in \boldsymbol{R}^{3}, t>0, \\
& \phi^{\varepsilon}(0, x, v)=\phi_{0}^{\varepsilon}(x, v), \quad x \in \mathscr{D}, v \in \boldsymbol{R}^{3}, \\
& -\hat{n} \cdot v \phi^{\varepsilon}(t, x, v)=g^{\varepsilon}(t, x, v), \quad t>0, x \in \partial \mathscr{D}, \hat{n} \cdot v<0 .
\end{aligned}
$$

Here the time has been rescaled so that in the $\varepsilon \rightarrow 0$ asymptotic limit the effects due to scalling in the equation balance in a nontrivial fashion. 
The data $\phi_{0}^{\varepsilon}$ and $g^{\varepsilon}$ depend on $\varepsilon$ in general. The basic problem is to study the behavior of $\phi^{\varepsilon}$ as $\varepsilon \rightarrow 0$ and obtain asymptotic expansions.

The geometry of the above problem, where the coefficients vary rapidly in a periodic manner, is referred to as the cellular geometry. The limit we seek to analyze is the homogenization limit, so called because the first approximation usually satisfies an equation with spatially averaged, i.e. homogenized, coefficients. In addition, a small mean free path limit or a diffusion limit is superimposed in (1.4.2). A description of the above problem can be found in [5].

The results of Section 3 show that the solution $\phi^{\varepsilon}$ of (1.4.2) can be constructed by solving homogenized diffusion equations and half-space problems. ${ }^{\dagger}$ The probabilisitc formulation assists in finding natural hypotheses for the validity of the expansions and yields more insight into the structure of the limits.

The reflecting boundary value problem $(1.2 .4),(1.2 .5)$ is scaled the same way. The boundary reflection function $B$ will in general depend on $\varepsilon$ now, and, it will turn out later, the principal term of $B$ must be totally reflecting, i.e. $\sigma_{B} \equiv 1$ in $(1.2 .6)$.

The interface problem (1.2.8)-(1.2.11) with the scaling of (1.4.2) remains intact and it is, in fact, a very important problem in nuclear engineering where the two media $\mathscr{D}_{1}$ and $\mathscr{D}_{2}$ are the core and the moderator (or reflector) respectively. The core is a problem with rapid periodic spatial variations while in the moderator only a diffusion approximation is sought.

Force fields can also exist. In general linear transport theory most problems of interest lead to equations which have abstractly the form

$$
\frac{\partial \phi^{\varepsilon}}{\partial t}=\frac{1}{\varepsilon^{2}} \mathcal{L}_{1} \phi^{\varepsilon}+\frac{1}{\varepsilon} \mathcal{L}_{2} \phi^{\varepsilon}+\mathcal{L}_{3} \phi^{\varepsilon}, \quad \phi^{\varepsilon}(0)=\phi_{0},
$$

where $\mathcal{L}_{1}, \mathcal{L}_{2}$ and $\mathcal{L}_{3}$ are linear operators. The asymptotic analysis of Section 3 applies formally to any such problem provided some general conditions are supposed to hold. It is more difficult however to find simple sufficient conditions for the validity of the formal expansions.

\footnotetext{
${ }^{\dagger}$ In Section 3 we do not consider cellular structure and boundary layers simultaneously.
} 


\section{5. Half-Space Problems}

Half-space problems are perhaps the only nontrivial boundary value problems that admit reasonably explicit analytical solutions. They have been studied extensively $[9,11]$ by essentially two different methods. The first is invariant imbedding [9] which lends itself readily to numerical analysis. The second is by eigenfunction expansion [11] where a careful analysis is needed to obtain the necessary orthogonality relations.

From the viewpoint of asymptotic analysis centered about the diffusion approximation, half-space problems enter in the boundary layer analysis. Here the diffusion solution is matched to that of an appropriately localized half-space problem. However, a relatively small portion of the full solution of the half-space problem enters the asymptotic formulas and this is an additional simplifying feature.

We consider the half-space problem in connection with the diffusion approximation without homogenization. It is time-homogeneous and under the additional hypothesis that the velocities take values on the unit sphere we have that $\phi=\phi(x, \mu), x \leq 0,-1 \leq \mu \leq 1$, ( $\mu=x$-component of velocity) satisfying

$$
\begin{aligned}
& \mu \frac{\partial \phi}{\partial x}=\int_{-1}^{1} \Sigma\left(\mu, \mu^{\prime}\right) \phi\left(x, \mu^{\prime}\right) d \mu^{\prime}-\sigma(\mu) \phi, \\
& x<0,-1 \leq \mu \leq 1,-\mu \phi(0, \mu)=\psi(\mu),-1 \leq \mu \leq 0 .
\end{aligned}
$$

Perturbation theory requires, in addition, that

$$
\int \Sigma\left(\mu^{\prime}, \mu\right) d \mu^{\prime}=\sigma(\mu)
$$

i.e. the half-space problem is conservative. If (1.5.2) is not satisfied, the asymptotic expansions have a degenerate form which is less interesting (and actually simpler to analyze) than when (1.5.2) holds.

We require the following qualitative properties from (1.5.1) under (1. 5. 2):

(i) The limit $\lim _{x \rightarrow-\infty} \phi(x, \ell)$ exists for each

$$
\phi(\mu) \geq 0 \quad\left(\int_{-1}^{0} \psi(\mu)<\infty\right)
$$

and is independent of $/ \iota$, 
(ii) This constant limit is approached exponentially fast.

For the actual formulas we would like to have explicit results for (1. 5. 4.)

(i) The limit of $\phi(x, \ell)$, as $x \rightarrow-\infty$, as a functional of $\psi(\mu)$,

(ii) The emergent distribution $\phi(0, \iota), 0 \leq \mu \leq 1$ as a functional of $\psi(\mu)$.

For general $\Sigma$ and $\sigma$ it is hardly surprising that explicit results in (1.5.4) are not available. On the other hand properties (1.5.3) are valid under very general conditions and this is, in fact, the subject of ergodic theory that is taken up in Sections 2.5-2.7. Probabilistic methods are well suited for the analysis of general qualitative properties.

The two quantities (i) and (ii) in (1.5.4) are related in a simple manner, assuming (1.5.2) and (1.5.3) hold. Let us restrict attention to the symmetric case

$$
\Sigma\left(\mu, \iota^{\prime}\right)=\Sigma\left(\mu^{\prime}, \iota^{\prime}\right), \quad-1 \leq \mu, \iota^{\prime} \leq 1,
$$

and derive this relation. Clearly, in view of (1.5.2) $\phi=1$ is a solution of (1.5.1) (without boundary conditions) and since $\int_{-1}^{1} \mu d \mu=0$

$$
\int_{-1}^{1} \Sigma\left(\mu, \mu^{\prime}\right) \gamma\left(\mu^{\prime}\right) d \mu^{\prime}-\sigma(\mu) \gamma(\mu)=\mu, \quad-1 \leq \mu \leq 1
$$

has a solution $\gamma(\mu)$, unique up to an additive constant. We are assuming that the Fredholm alternative applies to the operator on the right of (1.5.6). Along with $\phi=1$ we have now another solution $\phi=x+\gamma(\mu)$. Elementary manipulations yield thus the identities

$$
\begin{aligned}
& \int_{-1}^{1} \mu(\phi(0, \mu)-\bar{\phi}) d \mu=0 \\
& \int_{-1}^{1} \mu(\phi(0, \mu)-\bar{\phi}) \gamma(\mu) d \mu=0
\end{aligned}
$$

where $\bar{\phi}$ is defined by

$$
\bar{\phi}=\lim _{x \rightarrow-\infty} \phi(x, \mu)
$$

and is independent of " in view of (1.5.3).

The indentity (1.5.8) now yields the desired relation

$$
\bar{\phi}=\left(\int_{-1}^{1} \mu \gamma(\mu) d \mu\right)^{-1}\left(-\int_{-1}^{0} \gamma(\mu) \phi(\mu) d \mu+\int_{0}^{1} \mu \gamma(\mu) \phi(0, \mu) d \mu\right) .
$$


We note that the nonuniqueness of $\gamma(\mu)$ does not affect the value of $\bar{\phi}$ as one can verify using the identity (1.5.7). Thus, it is enough to have access to $\mu \phi(0, \mu), 0 \leq \mu \leq 1$, in order to construct the relevant asymptotic expansion. This quantity is perhaps most easily obtained by invariant imbedding [9]. We outline this procedure here.

Solving the problem (1.5.1) in the region $-\infty<x<y$ with the boundary conditions

$$
-\mu \phi(y, \mu)=\psi(\mu), \quad-1 \leq \mu \leq 0,
$$

does not alter, clearly, the quantity $\mu \phi(y, \mu), 0 \leq \mu \leq 1$, which is the emergent flux distribution. We shall exploit this fact to obtain a functional equation for the operator $R$ transforming the incident flux at $y$ to the reflected flux at $y$

$$
\text { (1. 5. 12) } \mu \phi(y, \mu)=-\int_{-1}^{0} R\left(\mu, \mu^{\prime}\right) \mu^{\prime} \phi\left(y, \mu^{\prime}\right) d \mu^{\prime}, \quad 0 \leq \mu \leq 1 .
$$

Actually, it is more convenient to introduce special notation and define $R$ in a slightly different manner. Let

$$
\begin{aligned}
& \phi^{+}(x, \iota)=\phi(x, \iota), \sigma^{+}(\mu)=\sigma(\ell) \\
& \phi^{-}(x, \mu)=\phi(x,-\mu), \sigma^{-}(\mu)=\sigma(-\mu), \quad 0 \leq \mu \leq 1,
\end{aligned}
$$

and

$$
\begin{aligned}
\Sigma^{++}\left(\mu, \mu^{\prime}\right)= & \Sigma\left(\mu, \mu^{\prime}\right) \\
\Sigma^{--}\left(\mu, \mu^{\prime}\right)= & \Sigma\left(-\mu,-\mu^{\prime}\right) \\
\Sigma^{+-}\left(\mu, \mu^{\prime}\right)= & \Sigma^{-+}\left(\iota^{\prime}, \mu\right)=\Sigma\left(\mu,-\mu^{\prime}\right)=\Sigma\left(-\mu^{\prime}, \mu\right), \\
& 0 \leq \mu, \mu^{\prime} \leq 1 .
\end{aligned}
$$

Then (1.5.1) takes the form

$$
\begin{aligned}
\mu \frac{\partial \phi^{+}}{\partial x}= & \int_{0}^{1} \Sigma^{++}\left(\mu, \mu^{\prime}\right) \phi^{+}\left(x, \mu^{\prime}\right) d \mu^{\prime} \\
& +\int_{0}^{1} \Sigma^{+-}\left(\mu, \mu^{\prime}\right) \phi^{-}\left(x, \mu^{\prime}\right) d \mu^{\prime}-\sigma^{+}(\mu) \phi^{+}(x, \mu) \\
-\mu \frac{\partial \phi^{+}}{\partial x}= & \int_{0}^{1} \Sigma^{-+}\left(\mu, \mu^{\prime}\right) \phi^{+}\left(x, \mu^{\prime}\right) d \mu^{\prime}
\end{aligned}
$$




$$
\begin{gathered}
+\int_{0}^{1} \Sigma^{--}\left(\mu, \mu^{\prime}\right) \phi^{-}\left(x, \mu^{\prime}\right) d \mu^{\prime}-\sigma^{-}(\mu) \phi^{-}(x, \mu) \\
x<0,0 \leq \mu \leq 1, \\
\iota \phi^{-}(0, \mu)=-\psi(-\mu), \quad 0 \leq \mu \leq 1 .
\end{gathered}
$$

We also define $R$, for any $x \leq y$,

$$
\mu \phi^{+}(y, \mu)=\int_{0}^{1} R\left(\mu, \mu^{\prime}\right) \phi^{-}\left(y, \mu^{\prime}\right) d \mu^{\prime}, \quad 0 \leq \mu \leq 1 .
$$

Differentiating this equation with respect to $y$, using (1.5.15) and the definition (1.5.16) we arrive at the following nonlinear integral equation for $R\left(\mu, \mu_{0}\right) \quad[9$, Chapter IV].

$$
\begin{aligned}
& \left(\frac{\sigma^{+}(\mu)}{\mu}+\frac{\sigma^{-}\left(\mu_{0}\right)}{\mu_{0}}\right) R\left(\mu, \mu_{0}\right) \\
& =\Sigma^{+-}\left(\mu, \mu_{0}\right)+\int_{0}^{1} \Sigma^{++}\left(\mu, \mu^{\prime}\right) \frac{R\left(\mu^{\prime}, \mu_{0}\right)}{\mu^{\prime}} d \mu^{\prime} \\
& \quad+\int_{0}^{1} \frac{R\left(\mu, \mu^{\prime}\right)}{\mu^{\prime}} \Sigma^{--}\left(\mu^{\prime}, \mu_{0}\right) d \mu^{\prime} \\
& \quad+\int_{0}^{1} \int_{0}^{1} \frac{R\left(\mu, \mu^{\prime}\right)}{\mu^{\prime}} \Sigma^{-+}\left(\mu^{\prime}, \mu^{\prime \prime}\right) \frac{R\left(\mu^{\prime \prime}, \mu_{0}\right)}{\mu^{\prime \prime}} d \mu^{\prime} d \mu^{\prime \prime}, \\
& 0 \leq \mu, \mu_{0} \leq 1 .
\end{aligned}
$$

Despite the difficult-looking form of (1.5.17), it is well suited for numerical integration especially by solving an initial value problem whose steady solution is $R\left(\mu, \mu_{0}\right)$ of (1.5.17).

It is worthwhile to specialize (1.5.1) and (1.5.17) to the isotropic case:

$$
\Sigma\left(\mu, \mu^{\prime}\right) \equiv \frac{1}{2}, \quad \sigma(\mu)=1
$$

Then (1.5.17) becomes

$$
\begin{aligned}
& \left(\frac{1}{\mu}+\frac{1}{\mu_{0}}\right) R\left(\mu, \mu_{0}\right) \\
& \quad=\frac{1}{2}\left(1+\int_{0}^{1} \frac{R\left(\mu, \mu^{\prime}\right)}{\iota^{\prime}} d \mu^{\prime}\right)\left(1+\int_{0}^{1} \frac{R\left(\mu^{\prime}, \mu_{0}\right)}{\mu^{\prime}} d \mu^{\prime}\right)
\end{aligned}
$$

and hence $R\left(\mu, \mu_{0}\right)=R\left(\mu_{0}, \mu\right)$. Letting 


$$
H(\mu)=1+\int_{0}^{1} \frac{R\left(\mu, \mu^{\prime}\right)}{\mu^{\prime}} d \mu^{\prime}=1+\int_{0}^{1} \frac{R\left(\mu^{\prime}, \mu\right)}{\mu^{\prime}} d \mu^{\prime}
$$

we find that $H(\mu)$ is Chandrasekhar's $H$ function $[9$, p. 97] which satisfies the nonlinear integral equation

$$
H(\mu)=1+\frac{1}{2} \mu H(\mu) \int_{0}^{1} \frac{H\left(\mu^{\prime}\right)}{\mu+\mu^{\prime}} d \mu^{\prime} .
$$

The values of this function and many identities are given in [9, Chapter $\mathrm{V}$ and Table XI, p. 125 with $\left.\omega_{0}=1\right]$.

In the isotropic case $\gamma(\mu)$ of $(1.5 .6)$ is equal to " $/$ so that (1.5.10) becomes

$$
\begin{aligned}
\bar{\phi} & =\frac{3}{2}\left[\int_{0}^{1} \mu^{\prime} \psi\left(-\mu^{\prime}\right) d \mu^{\prime}+\int_{0}^{1} \mu \mu \phi(0, \mu) d \mu\right] \\
& =\frac{3}{2}\left[\int_{0}^{1} \mu^{\prime} \psi\left(-\mu^{\prime}\right) d \mu^{\prime}+\int_{0}^{1} \mu \int_{0}^{1} R\left(\mu, \mu^{\prime}\right) \psi\left(-\mu^{\prime}\right) d \mu^{\prime} d \mu\right] \\
& =\frac{3}{2} \int_{0}^{1}\left[1+\frac{1}{2} \frac{H\left(\mu^{\prime}\right)}{\mu^{\prime}} \int_{0}^{1} \frac{\mu^{2} H(\mu) d \mu}{\mu+\mu^{\prime}}\right] \mu^{\prime} \psi\left(-\mu^{\prime}\right) d \mu^{\prime} .
\end{aligned}
$$

On using equation 22 of $[9$, p. 109] it follows that

$$
\bar{\phi}=\frac{\sqrt{3}}{2} \int_{0}^{1} H\left(\mu^{\prime}\right) \psi\left(-\mu^{\prime}\right) d \mu^{\prime} \text {. }
$$

We have therefore, in the isotropic case, an explicit expression for $\bar{\phi}$ which is a basic quantity for the asymptotic analysis.

The isotropic case is not the only one that allows the reduction via $H$ functions. What is necessary is that $\Sigma\left(\mu, \mu^{\prime}\right)$ be a degenerate kernel, i.e., a sum of products of functions of $\mu$ and $\mu^{\prime}$ (as well as symmetric). The analysis of the general $H$ function can be found in [9, Chapter V].

Problems with reflecting boundary conditions can be treated in a similar manner. Since we shall reconsider these problems in a probabilistic setting in Sections 2.6-2.7, we shall not discuss them further here. The sample of methods described above is intended to show that the boundary layer problems (half-space problem) that will appear in the constructions of Section 3 are far from intractable and a good deal is known about their effective solution. 


\section{§ 2. Probabilistic Theory of Transport Processes}

\section{1. Construction of Transport Processes}

Transport processes constitute a special class of Markov processes that are useful in modeling a variety of phenomena including the ones contemplated in the previous sections. We shall outline the construction of these processes here and we shall discuss some of their properties in the following sections. The probabilistic formulation of the asymptotic problems is in many ways more convenient than the corresponding one of the physical theory. The formal structure of the expansions differs, however, only insofar as the probabilistic approach deals with backward equations which are conservative while the physical approach deals with forward equations that are not necessarily (pointwise) conservative. ${ }^{\dagger}$

We shall consider a pair of processes $(X(t), Y(t)), t \geq 0$, with values in $\mathbb{R}^{n} \times \mathbb{R}^{m}$ which is constructed as follows. Let $(\xi(t), \eta(t))$ denote the solution of the deterministic system of ordinary differential equations

$$
\begin{aligned}
& \frac{d \xi(t)}{d t}=F(\xi(t), \eta(t)), \quad \xi(0)=x, \\
& \frac{d \eta(t)}{d t}=H(\xi(t), \eta(t)), \quad \eta(0)=y .
\end{aligned}
$$

We shall assume that $F$ and $H$ are smooth and bounded vector functions so that (2.1.1) has a solution for all $t \geq 0$. To indicate dependence on initial conditions we shall also write $\xi(t)=\xi(t, x, y), \eta(t)=\eta(t, x, y)$. Let $q(x, y)$ be a nonnegative smooth and bounded function and let $\tau_{1}$ be an exponentially distributed random variable so that

$$
P\left\{\tau_{1}>t\right\}=\exp \left(-\int_{0}^{t} q(\xi(s), \eta(s)) d s\right)
$$

In the interval $0 \leq t<\tau_{1}$ we define $(X(t), Y(t))$ by

$$
\begin{aligned}
& X(t)=\xi(t), \\
& Y(t)=\eta(t), \quad 0 \leq t<\tau_{1} .
\end{aligned}
$$

t To deal with non-conservative equations probabilistically one must consider branching transport processes. We shall not do this here (cf. [21]). 
At time $\tau_{1}$ the $Y$ process jumps to a new value and thereafter the motion continues along solutions of (2.1.1). Let $\pi(x, y, A), x \in \boldsymbol{R}^{n}, y \in \boldsymbol{R}^{m}$, $A \subset \mathbb{R}^{m}$ be a probability measure for each $x$ and $y$ and a continuous function for each Borel subset $A \subset \mathbb{R}^{m}$. Then we define

$$
\begin{aligned}
P\left\{\tau_{1} \leq\right. & \left.t, Y\left(\tau_{1}\right) \in A\right\} \\
= & \int_{0}^{t} \pi(\xi(s), \eta(s), A) q(\xi(s), \eta(s)) \\
& \quad \times \exp \left(-\int_{0}^{s} q(\xi(\gamma), \eta(\gamma)) d \gamma\right) d s .
\end{aligned}
$$

Let $\tau_{2}$ be an exponentially distributed random variable such that (2. 1.5) $P\left\{\tau_{2}>t\right\}$

$$
=\exp \left(-\int_{0}^{t} q\left(\xi\left(s, X_{1}, Y_{1}\right), \eta\left(s, X_{1}, Y_{1}\right)\right) d s\right), \quad X_{1}, Y_{1} \text { given },
$$

where $X_{1}=X\left(\tau_{1}\right)=\xi\left(\tau_{1}\right)$ and $Y_{1}=Y\left(\tau_{1}\right)$, the value immediately after the jump. In the interval $\tau_{1} \leq t<\tau_{1}+\tau_{2}$ we define $(X(t), Y(t))$ by

$$
\begin{aligned}
& X(t)=\xi\left(t, X_{1}, Y_{1}\right), \\
& Y(t)=\eta\left(t, I_{1}, I_{1}\right), \quad \tau_{1} \leq t<\bar{\nabla}_{1}+\tau_{2} .
\end{aligned}
$$

The process is now continued in the obvious manner. If it is uniquely defined then the resulting $(X(t), Y(t))$ is a Markov process which is a consequence of the exponential distribution of the interjump times. For uniqueness we must show that with probability one only finitely many jumps occur in each finite time interval. The uniqueness of solutions of (2.1.1) takes care of the rest. Assuming that

$$
0 \leq q \leq M<\infty
$$

we have that for $\alpha>0$

$$
\begin{aligned}
& P\left\{\tau_{1}+\tau_{2}+\cdots+\tau_{n} \leq t\right\} \\
& =P\left\{e^{-\alpha\left(\tau_{1}+\cdots+\tau_{n}\right)} \geq e^{-\alpha t}\right\} \leq e^{\alpha t} E\left\{e^{-\alpha\left(\tau_{1}+\cdots+\tau_{n}\right)}\right\} \\
& =e^{\alpha t} E\left\{e^{-\alpha\left(\tau_{1}+\cdots+\tau_{n-1}\right)} E\left\{e^{-\alpha \tau_{n}} \mid X\left(\widetilde{\tau}_{n-1}\right), Y\left(\widetilde{\tau}_{n-1}-\right)\right\}\right. \\
& \quad \leq e^{\alpha t}\left(\frac{M}{\alpha}\right)^{n}, \quad \tau_{n}=\tau_{1}+\cdots+\tau_{n} .
\end{aligned}
$$

Choosing $a$ so that $M \alpha^{-1}<1$ and letting $n \rightarrow \infty$ we find that indeed 
$\tau_{1}+\cdots+\tau_{n} \rightarrow \infty$ as $n \rightarrow \infty$ with probalbility one. Thus $(X(t), Y(t))$ is well defined and it is easily seen that it is a right-continuous $(X(t)$ is continuous) Feller process and hence a strong Markov process. We shall denote by $P_{r, y}$ and $E_{x, y,}$ the probability measure and the expectation of this process, starting at $(x, y)$. This measure is in the space of trajectories in $\boldsymbol{R}^{n+m}$ with continuous first $n$ components and right-continuous remaining $m$ components having left hand limits. The right-continuous $\sigma$-algebra of events up to time $t$ is denoted by $\mathscr{E}_{t}, t \geq 0$.

Let $f(x, y)$ be a bounded measurable function on $\boldsymbol{R}^{n} \times \mathbb{R}^{m}$ and define uby

$$
u(t, x, y)=E_{x, y}\{f(X(t), Y(t))\} .
$$

By conditioning on the first jump time $\bar{\sigma}_{1}$ we obtain the following integral equation for $u$ :

(2.1.10) $u(t, x, y)$

$$
\begin{aligned}
& =f(\xi(t), \eta(t)) \exp \left(-\int_{0}^{t} q(\xi(s), \eta(s)) d s\right) \\
& \quad+\int_{0}^{t} \int u(t-s, \xi(s), \approx) \pi(\xi(s), \eta(s), d \approx) q(\xi(s), \eta(s)) \\
& \quad \times \exp \left(-\int_{0}^{s} q(\xi(\gamma), \eta(\gamma)) d \gamma\right) d s .
\end{aligned}
$$

It is easy to see, independently of previous considerations, that (2.1.10) has a unique solution and that

$$
|u(t, x, y)| \leq \sup _{x, y}|f(x, y)| .
$$

This is done by the usual iteration arguments.

Under additional smoothness conditions on $f$, (2.1.10) can be written as a differential equation, as can be easily rerified.

$$
\begin{aligned}
& \frac{\partial u(t, x, y)}{\partial t}=\mathcal{L} u(t, x, y), \quad t>0, \\
& u(0, x, y)=f(x, y),
\end{aligned}
$$

where

$$
\mathcal{L g}(x, y) \equiv F(x, y) \cdot \frac{\partial y(x, y)}{\partial x}
$$




$$
+H(x, y) \cdot \frac{\partial g(x, y)}{\partial y}+Q_{x} g(x, y),
$$

(2.1.14) $\quad Q_{x} g(x, y) \equiv q(x, y) \int g(x, z) \pi(x, y, d z)-q(x, y) g(x, y)$.

The operator $\mathcal{L}$ is the infinitesimal generator of the Markov process $(X(t), Y(t))$ and it is the sum of two terms: The streaming terms along the vector fields $F$ and $H$ (here $F \cdot \frac{\partial}{\partial x}$ stands for $F$ dotted with the gradient operator) and the jump operator $Q$. For each fixed $x, Q_{x}$ is itself the generator of a Markov process, a pure jump process. We sometimes refer to this process as $Y_{x}(t)$. From (2.1.9), uniqueness and the Markov property it follows that the process $(X(t), Y(t))$ is completely determined by its generator $\mathcal{L}$ in (2.1.13). Equation (2.1.12) is the backward Kolmogorov equation.

Let $g(t, x, y)$ be a bounded continuous function such that

$$
\left(\frac{\partial}{\partial t}+\mathcal{L}\right) g(t, x, y)
$$

is also bounded and continuous. Define $M_{g}(t)$ by

(2.1.15) $\quad M_{g}(t)=g(t, X(t), Y(t))-\int_{0}^{t}\left(\frac{\partial}{\partial s}+\mathcal{L}\right) g(s, X(s), Y(s)) d s$.

From the fact that the expectation in (2.1.9) satisfies (2.1.10), and hence (2.1.12), it follows that

$$
E_{x, y}\left\{M_{g}(t)\right\}=g(0, x, y) .
$$

Using (2.1.16) it is easily verified now that $M_{g}(t)$ is a right-continuous martingale bounded for each $t<\infty$ :

$$
E\left\{M_{g}(t) \mid \mathscr{F}_{s}\right\}=M_{g}(s), \quad 0 \leq s \leq t .
$$

We may also write (2.1.15) in the form

(2.1.18) $g(t, X(t), Y(t))$

$$
=g(0, x, y)+\int_{0}^{t}\left(\frac{\partial}{\partial s}+\mathcal{L}\right) g(s, X(s), Y(s)) d s+\widetilde{M}_{g}(t),
$$

where $\widetilde{M}_{g}(t)=M_{g}(t)-g(0, x, y)$ is a zero-mean martingale. 


\section{2. Boundary Conditions}

Let $\mathscr{D}$ be a bounded open set in $\boldsymbol{R}^{n}$ with smooth boundary and let $t_{\mathscr{D}}$ be the first time $X(t)$ reaches ${ }^{\dagger} \partial \mathscr{D}$, the boundary of $\mathscr{D}$, starting from $(x, y), x \in \mathscr{D}$. We recall that $X(t)$ is continuous. Clearly $t_{\mathscr{D}}$ is a stopping time i.e. the event $\left\{t_{\mathscr{D}}>t\right\}$ belongs to $\mathscr{F}_{t}$ for all $t \geq 0$. The minimum of $t_{\mathscr{D}}$ and $t, t \wedge t_{\mathscr{D}}$, is also a stopping time.

Let $u(t, x, y)$ be any bounded continuous solution of

$$
\frac{\partial u}{\partial t}=\mathcal{L} u, \quad t>0, x \in \mathscr{D}, y \in \mathbb{R}^{m} .
$$

Then $u(t-s, X(s), Y(s)), 0 \leq s \leq t$, is a bounded, right-continuous martingale and by the optional stopping theorem [17]

$$
\left.E_{x, y}\left\{u\left(t-t \wedge t_{\mathscr{D}}\right), X\left(t \wedge t_{\mathscr{D}}\right), Y\left(t \wedge t_{\mathscr{D}}\right)\right)\right\}=u(t, x, y) .
$$

This identity can be rewritten in the form

$$
\begin{aligned}
u(t, x, y)= & E_{x, y}\left\{u(0, X(t), Y(t)), t \leq t_{\mathscr{D}}\right\} \\
& +E_{x, y}\left\{u\left(t-t_{\mathscr{D}}, X\left(t_{\mathscr{D}}\right), Y\left(t_{\mathscr{D}}\right)\right), t>t_{\mathscr{D}}\right\} .
\end{aligned}
$$

If now $u(t, x, y)$ is a solution of (2.2.1) subject to the boundary conditions

$$
\begin{array}{ll}
u(0, x, y)=f(x, y) & x \in \mathscr{D}, y \in \mathbb{R}^{m} \\
u(t, x, y)=h\left(x, y^{\prime}\right) & x \in \partial \mathscr{D}, y \in\{J: \hat{n}(x) \cdot F(x, J) \geq 0\}
\end{array}
$$

it follows that ( $f$ and $h$ are bounded measurable)

$$
\begin{aligned}
u(t, x, y)= & E_{x, y}\left\{f(X(t), Y(t)), t \leq t_{\mathscr{D}}\right\} \\
& +E_{x, y}\left\{h\left(X\left(t_{\mathscr{D}}\right), Y\left(t_{\mathscr{D}}\right)\right), t>t_{\mathscr{D}}\right\} .
\end{aligned}
$$

In (2. 2. 4) $\hat{n}$ denotes the unit outward normal to $\partial \mathscr{D}$ and the restriction on $y$ will be denoted more compactly by $\hat{n} \cdot F \geq 0$. It is clear that at the instant $X(t)$ touches the boundary, its velocity $F(X(t), Y(t))$ is pointing outward.

The expression (2.2.5) is well defined since the measure $P_{x, y}$ of the process is well defined and $f$ and $h$ are bounded and measurable. It is

\footnotetext{
${ }^{+} t_{\mathscr{D}}=+\infty$ if $X(t)$ never reaches $\left.\partial \mathscr{D}\right)$.
} 
necessary to verify that the function $u$ defined by (2.2.5) satisfies (2.2.1) and (2.2.4) in an appropriate sense. By a renewal argument similar to the one used in the previous section it follows that $u$ of (2.2.5) satisfies the integral equation

$$
\begin{aligned}
u(t, x, y)= & \chi\left(t \leq \bar{t}_{\mathscr{D}}\right) f(\xi(t), \eta(t)) \\
& \times \exp \left(-\int_{0}^{t} q(\xi(s), \eta(s)) d s\right) \\
& +\chi\left(t>\bar{t}_{\mathscr{D}}\right) h\left(\xi\left(\bar{t}_{\mathscr{D}}\right), \eta\left(\bar{t}_{\mathscr{D}}\right)\right) \\
& \times \exp \left(-\int_{0}^{\bar{t}_{\mathscr{D}}} q(\xi(s), \eta(s)) d s\right) \\
& +\int_{0}^{t \wedge \bar{t}_{\mathscr{D}}} \int u(t-s, \hat{\xi}(s), z) \pi(\xi(s), \eta(s), d z) \\
& \times q(\xi(s), \eta(s)) \exp \left(-\int_{0}^{s} q(\xi(\gamma), \eta(\gamma)) d \gamma\right) d s,
\end{aligned}
$$

where $\bar{t}_{\mathscr{D}}$ is the first time $\xi(t)$ reaches $\partial \mathscr{D}$ starting from $(x, y), x \in \mathscr{D}$. We assume here that $f$ and $h$ are compatible

$$
f\left(x, y^{\prime}\right)=h(x, y), x \in \partial \mathscr{D}, \quad \hat{n} \cdot F(x, y) \geq 0,
$$

and $\chi\left(t \leq \bar{t}_{\mathscr{D}}\right)=1$ if $t \leq \bar{t}_{\mathscr{D}}$ and zero otherwise.

It is easily verified now that (2.2.6) is the integral equation version of (2.2.1), (2.2.4). Therefore the necessary identification of the solution of the boundary value problem (2.2.1), (2.2.4), actually its integral equation form (2.2.6), with (2.2.5) is complete. Incidentally, the usual iteration arguments provide existence and uniqueness for (2.2.6) independently of previous considerations.

We consider the process with reflection. For each $x \in \partial \mathscr{D}$ let $S^{+}$ $=\{y: \hat{n}(x) \cdot F(x, y) \geq 0\}$ and $S^{-}=\{y: \hat{n}(x) \cdot F(x, y)<0\}$ and let $B(x, y, A)$, $x \in \partial \mathscr{D}), y \in S^{+}, A \subset S^{-}$be a stochastic kernel for each $x \in \partial \mathscr{D}$. This means that for each $x \in \partial \mathscr{D}, y \in S^{+}, B(x, y, A)$ is a probability measure on $S^{-}$(of total mass equal to 1 ) and $B\left(x, y, A \cap S^{-}\right)$is continuous in $x$ and $y$ for each Borel set $A$ in $\boldsymbol{R}^{m}$. For $x \in \mathscr{D}$ (D) is an open set), $y$ arbitrary, let the generator of our process be $\mathcal{L}$ of (2.1.13) as usual. For $x \in \mathcal{O D}$ and $y \in S^{-}$again let $\mathcal{L}$ be the generator but for $x \in \mathscr{D Q D}$ and $y \in S^{+}$let the generator be given by 


$$
\begin{gathered}
(B-I) g(x, y)=\int_{S^{-}} B(x, y, d z) g(x, z)-g(x, y), \\
x \in \partial \mathscr{D}, y \in S^{+}
\end{gathered}
$$

More precisely, the process proceeds as usual in the interior or at boundary points with inward pointing velocity but, upon hitting the boundary with outgoing velocity, its velocity is instantaneously switched to an ingoing one according to the probability law $B(x, y, \mathrm{~A})$.

To insure uniqueness, and hence the Markov property, it is enough to show that in any finite time interval the process returns to the boundary finitely often with probability one. We need for this one additional assumption as follows. For each $\varepsilon>0$ there exists a $\delta>0$ such that

$$
\inf _{x \subseteq \partial \mathscr{D}} \inf _{y \in S^{+}} \int_{\grave{n} \cdot F<-\varepsilon} B(x, y, d \approx) \geq \delta
$$

Let $\tau_{1}, \tau_{2}, \cdots$ be the successive time intervals between returns to the boundary. For any $\alpha>0$ we have

$$
\begin{gathered}
P_{x, y}\left\{\tau_{1}+\tau_{2}+\cdots+\tau_{n} \leq t\right\} \leq e^{\alpha t} E_{x, y}\left\{e^{-\alpha\left(\tau_{1}+\cdots+\tau_{n}\right)}\right\} \\
=e^{\alpha t} E_{x, y}\left\{e^{-\alpha\left(\tau_{1}+\cdots+\tau_{n-1}\right)} E\left\{e^{-\alpha \tau_{n}} \mid X\left(\sigma_{n-1}\right), Y\left(\sigma_{n-1}\right)\right\}\right\}, \\
\sigma_{n}=\tau_{1}+\cdots+\tau_{n} .
\end{gathered}
$$

It suffices to show that for $\alpha$ sufficiently large

$$
E_{x . y}\left\{e^{-a t}\right\} \leq \beta<1
$$

where $x \in \partial \mathscr{D}$ and $y \in S^{-}$and $=$is the first return time to the boundary. This along with (2.2.10) implies that only finitely many returns occur in each finite time interval. But we have

$$
\begin{aligned}
1-E_{x, y}\left\{e^{-\alpha \cdot}\right\} & =\int_{S^{-}} B(x, y, d z) E_{x, z}\left\{1-e^{-\alpha *}\right\} \\
& \geq 0 \inf _{x \in \partial \mathscr{D}} \inf _{\hat{n} \cdot F<-\varepsilon} E_{x, y}\left\{1-c^{-\alpha *}\right\} .
\end{aligned}
$$

For $x \in \partial \mathscr{D}$ and $y$ such that $\hat{n}(x) \cdot F(x, y)<-\varepsilon$, it follows that $\tau \geq \hat{\tau} \wedge \tilde{\tau}$ where $\hat{\tau}$ is the time until the first jump of $Y$ after leaving $x$ and $\tilde{\tau}$ is the deterministic time it takes the path to reach $\partial \mathscr{D}$ starting from $(x, y)$. Clearly $\tilde{\tau}$ is positive uniformly in $x \in \partial \mathscr{D}$ and $y$ such that $\hat{n}(x) \cdot F(x, y)$ $<-\varepsilon$. Thus, 


$$
\begin{aligned}
E_{x, y}\left\{e^{-\alpha \tau}\right\} & \leq E_{x, y}\left\{e^{-\alpha \hat{\tau} \wedge \tilde{\tau}}\right\} \\
& \leq E_{x, y}\left\{e^{-\alpha \hat{\tau}}, \hat{\tau} \leq \tilde{\tau}\right\}+e^{-\alpha \tilde{\tau}} P_{x, y}\{\hat{\tau}>\tilde{\tau}\} \\
& \leq \frac{M}{\alpha}+e^{-\alpha \tilde{\tau}} .
\end{aligned}
$$

By choosing $\alpha$ large enough the right hand side of $(2.2 .13)$ can be made less than one uniformly in $x \in \partial \mathscr{D}$ and $y$ such that $\hat{n} \cdot F<-\varepsilon$. This combined with (2.2.12) yields (2.2.11).

Therefore the reflected process subject to (2.2.9) is uniquely defined, is a right continuous strong Markov process as before, and we will denote by $P_{x, y}^{R}$ its measure and $E_{x, y}^{R}$ expectation relative to this measure.

Let $f(x, y)$ be a bounded measurable function on $\mathscr{D} \times S$, where we define

$$
S=S^{\vdash} \cup S^{-},
$$

since we are also contemplating situations where $S$ is not $\boldsymbol{R}^{m}$ but some other space, a compact metric space, ${ }^{\dagger}$ say. Put

$$
u(t, x, y)=E_{x, y}^{R}\{f(X(t), Y(t))\} .
$$

In a manner analogous to $(2.2 .6)$, if we let $\bar{t}_{\mathscr{D}}$ be the first time to reach $\partial \mathscr{D}$ along the orbits of (2.1.1) starting from $(x, y), x \in \mathscr{D}$, it follows that

(2.2.16) $u(t, x, y)$

$$
\begin{aligned}
= & \chi\left(t \leq \bar{t}_{\mathscr{D}}\right) f(\xi(t), \eta(t)) \exp \left(-\int_{0}^{t} q(\xi(s), \eta(s)) d s\right) \\
& +\chi\left(\iota \bar{t}_{\mathscr{D}}\right) \int_{s^{-}} B\left(\xi\left(\bar{t}_{\mathscr{D}}\right), \eta\left(\bar{t}_{\mathscr{D}}\right), d z\right) u\left(t-\bar{t}_{\mathscr{D}}, \xi\left(\bar{t}_{\mathscr{D}}\right), z\right) \\
& \times \exp \left(-\int_{0}^{\bar{t}_{\mathscr{D}}} q(\xi(s), \eta(s)) d s\right) \\
& +\int_{0}^{t \wedge \bar{t}_{\mathscr{D}}} \int u(t-s, \xi(s), z) \pi(\xi(s), \eta(s), d z) \\
& \times q(\xi(s), \eta(s)) \exp \left(-\int_{0}^{s} q(\xi(\gamma), \eta(\gamma)) d \gamma\right) d s .
\end{aligned}
$$

\footnotetext{
The compactness will be used later in the ergodic theory and the asymptotic expan-
} sions. 
We know already that the solution of this equation exists and is unique. Existence follows also by the usual iteration arguments independently of the above considerations. It is not difficult to verify now that (2.2.16) is the integral equation version of the boundary value problem (defined when $f$ is smooth)

$$
\begin{gathered}
\frac{\partial u(t, x, y)}{\partial t}=\mathcal{L} u(t, x, y), \quad t>0, \\
u(0, x, y)=f(x, y), \quad x \in \mathscr{D}, y \in S, x \in \partial \mathscr{D}, y \in S^{-}, \\
u(t, x, y)=\int_{S^{-}} B(x, y, d z) u(t, x, z), \\
x \in \partial \mathscr{D}, t>0, y \in S^{+} .
\end{gathered}
$$

This problem is the backward Kolmogorov equation of the reflected process $(X(t), Y(t))$.

Let $G$ and $\partial G$ be defined as follows

$$
\begin{aligned}
& G=(\mathscr{D} \times S) \cup\left\{(x, y), x \in \partial \mathscr{D}, y \in S^{-}\right\}, \\
& \partial G=\left\{(x, y), x \in \partial \mathscr{D}, y \in S^{\top}\right\} .
\end{aligned}
$$

The reflected process is a process' on $\bar{G}=G \cup \partial G$. Let $g(t, x, y)$ be a bounded continuous function such that $\left(\partial_{t}+\mathcal{L}\right) g$ is also bounded and continuous on $[0, \infty) \times G$. Let $\chi_{G}(x, y)$ be the characteristic function of $G$ and let

$$
\begin{aligned}
M_{g}(t)=g(t, X(t), Y(t)) \\
-\int_{0}^{t} \%_{G}(X(s), Y(s))\left(\frac{\partial}{\partial s}+\mathcal{L}\right) g(s, X(s), Y(s)) d s \\
-\int_{0}^{t} \%_{\partial G}(X(s), Y(s-))[B g(s, X(s), Y(s-)) \\
\quad-g(s, X(s)), Y(s-))] d N(s) .
\end{aligned}
$$

Here $N(t), t \geq 0$ denotes the right-continuous increasing step process of unit jumps defined by

$$
N(t)=0, \quad \sigma_{0}=0 \leq t<\sigma_{1}=\sigma_{1},
$$

\footnotetext{
' Actually, the process spends zero time on $\partial G$ by construction.
} 


$$
\begin{aligned}
& N(t)=1, \quad \sigma_{1}=\tau_{1} \leq t<\tau_{1}+\tau_{2}=\sigma_{2}, \\
& \vdots \\
& N(t)=k, \quad \sigma_{k}=\tau_{1}+\cdots+\tau_{k} \leq t<\tau_{1}+\cdots+\tau_{k+1}=\sigma_{k-1},
\end{aligned}
$$

where $\tau_{1}$ is the first time $\partial \mathscr{D}$ is reached and $\tau_{2}, \tau_{3}, \cdots$, are the times between successive returns to the boundary. The process $N(t)$ increases only when $(X(t), Y(t))$ is in $\partial G$ but spends no time in $\partial G$ since it is instantaneously reflected; $N(t)$ is well defined since only finitely many jumps occur in each finite time interval. The integral with respect to $d N$ in (2.2.20) is an abbreviation for the expression

$$
-\sum_{k=1}^{\infty}(B-I) g\left(\sigma_{k}, X\left(\sigma_{k}\right), Y\left(\sigma_{k}-\right)\right) \chi\left(\sigma_{k} \leq t\right)
$$

where $Y\left(\sigma_{k}-\right)$ the left hand limit of the right continuous process $Y(t)$ at the instant $\partial \mathscr{D}$ is reached and before reflection. Paths with jumps times equal to the hitting times at the boundary occur with probability zero and are excluded from consideration.

We shall show that

$$
E_{x, y}^{R}\left\{M_{g}(t)\right\}=g(0, x, y) .
$$

From this it follows easily that $M_{g}(t)$ is a right-continuous martingale just as the $M_{g}(t)$ of (2.1.15) was a martingale. From (2.2.20) we have

$$
\begin{gathered}
M_{g}(t)=\sum_{k=1}^{\infty}\left[g\left(t \wedge \sigma_{k}, X\left(t \wedge \sigma_{k}\right), Y\left(t \wedge \sigma_{k}\right)\right)\right. \\
\left.-g\left(t \wedge \sigma_{k}, X\left(t \wedge \sigma_{k}\right), Y\left(t \wedge \sigma_{k}-\right)\right)\right] \\
+\sum_{k=1}^{\infty}\left[g\left(t \wedge \sigma_{k}, X\left(t \wedge \sigma_{k}\right), Y\left(t \wedge \sigma_{k}-\right)\right)\right. \\
\left.-g\left(t \wedge \sigma_{k-1}, X\left(t \wedge \sigma_{k-1}\right), Y\left(t \wedge \sigma_{k-1}\right)\right)\right] \\
+g(0, x, y)-\sum_{k=1}^{\infty} \int_{t \wedge \sigma_{k-1}}^{t \wedge \sigma_{k}-}\left(\frac{\partial}{\partial s}+\mathcal{L}\right) g(s, X(s), Y(s)) d s \\
-\sum_{k=1}^{\infty}(B-I) g\left(\sigma_{k}, X\left(\sigma_{k}\right), Y\left(\sigma_{k}-\right)\right) \psi\left(\sigma_{k} \leq t\right) .
\end{gathered}
$$

This identity holds for any $\sigma_{n} \leq t<\sigma_{n+1}, n=0,1,2, \cdots$, and the sums are over finitely many terms with probability one. We rearrange terms on the right as follows 


$$
\begin{aligned}
M_{g}(t)=\sum_{k=1}^{\infty}\left[g\left(t \wedge \sigma_{k}, X\left(t \wedge \sigma_{k}\right), Y^{r}\left(t \wedge \sigma_{k}-\right)\right)\right. & -g\left(t \wedge \sigma_{k-1}, X\left(t \wedge \sigma_{k-1}\right), Y^{r}\left(t \wedge \sigma_{k-1}\right)\right) \\
- & \left.\int_{t \wedge \sigma_{k-1}}^{t \wedge \sigma_{k-}}\left(\frac{\partial}{\partial s}+\mathcal{L}\right) g(s, X(s), Y(s)) d s\right] \\
+ & \sum_{k=1}^{\infty}\left[g\left(\sigma_{k}, X\left(\sigma_{k}\right), I^{r}\left(\sigma_{k}\right)\right)-g\left(\sigma_{k}, X\left(\sigma_{k}\right), Y\left(\sigma_{k}-\right)\right)\right. \\
& \left.\quad(B-I) g\left(\sigma_{k}, X\left(\sigma_{k}\right), Y^{r}\left(\sigma_{k}-\right)\right)\right] \chi\left(\sigma_{k} \leq t\right) \\
& +g(0, x, y) .
\end{aligned}
$$

Now we take expectations. We have

$$
\begin{aligned}
E_{x, y}^{R}\left\{M_{g}(t)\right\}= & g(0, x, y)+E_{x, y}^{R}\left\{\sum _ { k = 1 } ^ { \infty } E \left\{g\left(t \wedge \sigma_{k}, X\left(t \wedge \sigma_{k}\right), Y\left(t \wedge \sigma_{k}-\right)\right)\right.\right. \\
& -g\left(t \wedge \sigma_{k-1}, X\left(t \wedge \sigma_{k-1}\right), Y\left(t \wedge \sigma_{k-1}\right)\right) \\
& \left.\left.-\int_{t \wedge \sigma_{k-1}}^{t \wedge \sigma_{k}-}\left(\frac{\partial}{\partial s}+\mathcal{L}\right) g(s, \Gamma(s), Y(s)) d s \mid \mathscr{F}_{t, \backslash \sigma_{k-1}}\right\}\right\} \\
& +E_{x, y}^{R}\left\{\sum _ { k = 1 } ^ { \infty } E \left\{g\left(\sigma_{k}, X\left(\sigma_{k}\right), Y\left(\sigma_{k}\right)\right)\right.\right. \\
& \quad-g\left(\sigma_{k}, Y\left(\sigma_{k}\right), Y\left(\sigma_{k}-\right)\right) \\
& \left.\left.-(B-I) g\left(\sigma_{k}, X\left(\sigma_{k}\right), Y\left(\sigma_{k}-\right)\right) \mid \mathscr{F}_{\sigma_{k}-}\right\} \chi\left(\sigma_{k} \leq t\right)\right\} \\
= & g(0, x, y),
\end{aligned}
$$

which proves (2.2.22). The expectations inside the sums on the right are zero, the first by the optional stopping theorem for the martingale (2.1.15) in the interior of $\mathscr{D}$, and the second because of the definition (2.2.8) of the reflection operator

(2.2.23) $\quad E\left\{g\left(\sigma_{k}, \mathrm{X}\left(\sigma_{k}\right), Y\left(\sigma_{k}\right)\right) \mid \mathscr{F}_{\sigma_{k}-}\right\}=B g\left(\sigma_{k}, \mathrm{X}\left(\sigma_{k}\right), Y\left(\sigma_{k}-\right)\right)$.

We shall need in the next section a more elaborate version of the pair (2.2.20), (2.2.22) which we shall consider next.

Let $a(x, y)$ be a bounded continuous function on $\bar{G}$ and $b(x, y)$ a bounded continuous function on $\partial \mathscr{D} \times S$ with $b(x, y)<1$. Let $g(t, x, y)$ be as in (2.2.20) and define $U_{k}(t)$ and $\Gamma_{k}^{r}, k=1,2,3, \cdots$, as follows:

$$
U_{k}(t)=\exp \left\{\int_{t \wedge \sigma_{k-1}}^{t \wedge \sigma_{k-}} a(X(s), Y(s)) d s\right\}
$$




$$
\begin{array}{r}
\quad \times g\left(t \wedge \sigma_{k}, X\left(t \wedge \sigma_{k}\right), Y\left(t \wedge \sigma_{k}-\right)\right) \\
-g\left(t \wedge \sigma_{k-1}, X\left(t \wedge \sigma_{k-1}\right), Y\left(t \wedge \sigma_{k-1}\right)\right) \\
-\int_{t \wedge \sigma_{k-1}}^{t \wedge \sigma_{k-}} \exp \left\{\int_{t \wedge \sigma_{k-1}}^{s} a(X(\gamma), Y(\gamma)) d \gamma\right\} \\
\cdot\left(\frac{\partial}{\partial s}+\mathcal{L}+a\right) g(s, X(s), Y(s)) d s,
\end{array}
$$

$$
\begin{aligned}
V_{k}= & \frac{1}{1-b\left(X\left(\sigma_{k}\right), Y\left(\sigma_{k}-\right)\right)} g\left(\sigma_{k}, X\left(\sigma_{k}\right), Y\left(\sigma_{k}\right)\right) \\
& -g\left(\sigma_{k}, X\left(\sigma_{k}\right), Y\left(\sigma_{k}-\right)\right) \\
& -\frac{1}{1-b\left(X\left(\sigma_{k}\right), Y\left(\sigma_{k}-\right)\right)}(B-I+b) \\
& \times g\left(\sigma_{k}, X\left(\sigma_{k}\right), Y\left(\sigma_{k}-\right)\right), \quad k=1,2, \cdots .
\end{aligned}
$$

We note that, as above, for $k=1,2, \cdots$,

$$
E\left\{U_{k}(t) \mid \mathscr{F}_{t \wedge \sigma_{k-1}}\right\}=0, \quad E\left\{V_{k} \mid \mathscr{F}_{\sigma_{k}-}\right\}=0
$$

We now define

$$
\begin{aligned}
W(t)= & \sum_{k=1}^{\infty}\left[\prod_{j=1}^{k-1} \exp \left\{\int_{t \wedge \sigma_{j-1}}^{t \wedge \sigma_{j-}} a(X(s), Y(s)) d s\right\}\right. \\
& \cdot \prod_{j=1}^{k-1} \frac{1}{1-b\left(X\left(\sigma_{j}\right), Y\left(\sigma_{j}-\right)\right)} U_{k}(t) \\
& +\prod_{j=1}^{k} \exp \left\{\int_{t \wedge \sigma_{j-1}}^{t \wedge \sigma_{j-}} a(X(s), Y(s)) d s\right\} \\
& \left.\cdot \prod_{j=1}^{k-1} \frac{1}{1-b\left(X\left(\sigma_{j}\right), Y\left(\sigma_{j-}\right)\right)} V_{k} \chi\left(\sigma_{k} \leq t\right)\right] .
\end{aligned}
$$

This expression is well defined and the sum runs over finitely many terms with probability one. In view of (2.2.26) we have

$$
E_{x, y}^{R}\{W(t)\}=0 \text {. }
$$

By rearranging terms in (2.2.27) and cancelling several of them when using (2.2.24) and (2.2.25) we arrive at the identity

$$
\text { (2.2.29) } \begin{aligned}
E_{x, y}^{R} & \{A(t) g(t, X(t), Y(t)) \\
& -\int_{0}^{t} A(s)\left(\frac{\partial}{\partial s}+\mathcal{L}+a\right) g(s, X(s), Y(s)) d s
\end{aligned}
$$




$$
\begin{aligned}
& \left.-\int_{0}^{t} A(s)(B-I+b) g(s, X(s), Y(s-)) d N(s)\right\} \\
& =g(0, x, y)
\end{aligned}
$$

with

$$
\begin{aligned}
A(t)= & \exp \left[\int_{0}^{t} a(X(s), Y(s)) d s\right. \\
& \left.+\int_{0}^{t} \log \left(\frac{1}{1-b(X(s), Y(s-))}\right) d N(s)\right]
\end{aligned}
$$

When written out explicitly, the integrals with respect to $d N$ take the following form:

$$
\begin{aligned}
& \int_{0}^{t} \log \left(\frac{1}{1-b(X(s), Y(s-))}\right) d N(s) \\
& \quad=\sum_{k=1}^{\infty} \log \left(\frac{1}{1-b\left(X\left(\sigma_{k}\right), Y\left(\sigma_{k}-\right)\right)}\right) \psi\left(\sigma_{k} \leq t\right),
\end{aligned}
$$

$$
\begin{aligned}
\int_{0}^{t} A(s)(B-I+b) g(s, X(s), Y(s-)) d N(s) \\
=\sum_{k=1}^{\infty} \exp \left\{\int_{0}^{\sigma_{k-}} a(X(s), Y(s)) d s\right\} \prod_{j=1}^{k} \frac{1}{1-b\left(X\left(\sigma_{j}\right), Y\left(\sigma_{j}-\right)\right)} \\
\cdot(B-I+b) g\left(\sigma_{k}, X\left(\sigma_{k}\right), Y\left(\sigma_{k}-\right)\right) \chi\left(\sigma_{k} \leq t\right) .
\end{aligned}
$$

From the identity (2.2.29) we conclude that the expression under the expectation on the left side in (2.2.29) is a martingle.

\subsection{Connection with the Physical Theory}

The physical problems of linear conservative transport theory do not deal with the statistics of a single purticle but with the average density of a random number of them. Howvever, the motion of one particle does not affect the others (by linearity) so that all pertinent information can be obtained by integrating functionals of a single process relative to the measures already constructed and by integrating over the initial points with respect to appropriate measures given as data of the problem.

Consider first the problem without reflection. Let $a(x, y), f(x, y)$, $g(x, y)$ be bounded measurable functions on $G$ and let $h(x, y)$ be bounded 
measurable on $\partial G$. The generalized ${ }^{\dagger}$ solution of the boundary value problem

$$
\begin{gathered}
\frac{\partial u(t, x, y)}{\partial t}=\mathcal{L} u(t, x, y)+a(x, y) u(t, x, y)+f(x, y), \\
t>0, \quad(x, y) \in G \\
u(0, x, y)=g(x, y), \quad(x, y) \in G \\
u(t, x, y)=h(x, y), \quad(x, y) \in \partial G
\end{gathered}
$$

admits the probabilistic representation

$$
\begin{aligned}
u(t, x, y)= & E_{x, y}\left\{\exp \left(\int_{0}^{t} a(X(s), Y(s)) d s\right)\right. \\
& \left.\times g(X(t), Y(t)), t<t_{\mathscr{D}}\right\} \\
+ & E_{x, y}\left\{\exp \left(\int_{0}^{t} \mathfrak{D} a(X(s), Y(s)) d s\right)\right. \\
& \left.\times h\left(X\left(t_{\mathscr{D}}\right), Y\left(t_{\mathscr{D}}\right)\right), t \geq t_{\mathscr{D}}\right\} \\
+ & E_{x, y}\left\{\int_{0}^{t \wedge t_{\mathscr{D}}} \exp \left(\int_{0}^{\sigma} a(X(\gamma), Y(\gamma)) d \gamma\right)\right. \\
& \times f(X(\sigma), Y(\sigma)) d \sigma\} .
\end{aligned}
$$

Here $t_{\mathscr{D}}$ is the first time $X(t)$ reaches the boundary $\partial \mathscr{D}$ of $\mathscr{D}$ and the data $g$ and $h$ are assumed compatible at $x \in \partial \mathscr{D}, y \in S^{+}$. The representation (2.3.2) follows easily from a ininor modification of (2.1.16). In general, with compatible data or not, (2.3.2) is the probabilistically natural generalized solution of (2.3.1).

The expression (2.3.2) is the expectation of a functional of a single particle starting at $(x, y)$. If $G(x, y)$ is the initial density of particles, $F(t, x, y)$ is the density per unit time of particles created inside and $H(t, x, y)$ is the flux density of particles per unit time entering from the boundary (so that $x \in \partial \mathscr{D}, y \in S^{-}$) then the expectation of the functional in (2.3.2) over all particles is

\footnotetext{
t That is, the solution of the integral equation version of (2.3.1).
} 


$$
\begin{aligned}
& w(t)= \int_{\mathscr{D}} \int_{S} u(t, x, y) G(x, y) d x d y \\
&+\int_{0}^{t} \int_{\mathscr{D}} \int_{S} u(t-s, x, y) F(s, x, y) d s d x d y \\
&+\int_{0}^{t} \int_{\partial \mathscr{D}} \int_{S^{-}} u(t-s, x, y) H(s, x, y) d s d \widetilde{S}(x) d y, \\
& d \tilde{S}(x)=\text { surface element on } \partial \mathscr{D} .
\end{aligned}
$$

In particular, if $a=h=f=0$ in (2.3.2) and $g(x, y)=\chi_{A}(x)$ where $A$ is a subset of $\mathscr{D}$, say, then the corresponding $w(t)$ in (2.3.3) is the expected number of particles in $A$ at time $t$ that have not yet reached $\partial \mathscr{D}$. It is clear from this example that all relevant expectations can be constructed by the above 2-step procedure i.e., first by an expectation of the form (2.3.2) and then an average of the form (2.3.3). The connection with the forward equations corresponds to inserting $\delta$-functions in (2.3.2) for the functions $g, h$, or $f$ depending on the situation.

For the reflected process the situation is similar. Suppose $a, f, g$ and $h$ are as before and let $b(x, y)<1$ be another bounded measurable function on $\partial G$. The generalized, as above, solution of the boundary value problem

$$
\begin{gathered}
\text { (2.3.4) } \quad \begin{array}{c}
\frac{\partial u(t, x, y)}{\partial t}=\mathcal{L} u(t, x, y)+a(x, y) u(t, x, y)+f(x, y), \\
t>0, \quad(x, y) \in G \\
u(0, x, y)=g(x, y), \quad(x, y) \in G \\
\int_{S^{-}} B(x, y, d z) u(t, x, z)-u(t, x, y)+b(x, y) u(t, x, y) \\
+h(x, y)=0, \quad(x, y) \in \partial G
\end{array}
\end{gathered}
$$

admits the probabilistic representation ${ }^{\dagger}$

$$
\begin{aligned}
u(t, x, y)= & E_{x, y}^{R}\{A(t) g(X(t), Y(t))\} \\
& +E_{x, y}^{R}\left\{\int_{0}^{t} A(s) f(X(s), Y(s)) d s\right\} \\
& +E_{x, y}^{R}\left\{\int_{0}^{t} A(s) h(X(s), Y(s-)) d N(s)\right\}
\end{aligned}
$$

${ }^{\dagger}$ We drop $\chi_{G}$ and $\chi_{\partial G}$ since the process spends zero time on $\partial G$ anyway. 
where

$$
\begin{aligned}
A(t)= & \exp \left\{\int_{0}^{t} a(X(s), Y(s)) d s\right. \\
& \left.+\int_{0}^{\iota} \log \left(\frac{1}{1-b(X(s), Y(s-))}\right) d N(s)\right\} .
\end{aligned}
$$

Here we employ the notation of Section 2. 2. The representation (2.3.5) follows from (2.2.29) and, as before, it gives the probabilistically natural generalized solution to (2.3.4).

The functions $a$ and $\frac{1}{1-b}$ represent $^{\dagger}$ creation or annihilation (killing) rates for the process in the interior and the boundary, respectively, depending upon the sign of $a$ and $b$; negative corresponds to annihilation. Averages over collections of particles yield expressions such as (2.3.3) here also.

As an example, if $a=b=g=f=0$ and $h=1$ then

$$
u(t, x, y)=E_{x, y}^{R}\{N(t)\}
$$

is the expected number of times the process reaches $\partial \mathscr{D}$ up to time $t$ starting from $(x, y)$ and it satisfies the boundary value problem

$$
\begin{aligned}
& \frac{\partial u(t, x, y)}{\partial t}=\mathcal{L} u(t, x, y), \quad t>0, \quad(x, y) \in G \\
& u(0, x, y)=0, \quad(x, y) \in G \\
& u(t, x, y)=\int_{S^{-}} B(x, y, d z) u(t, x, y)+1, \quad(x, y) \in \partial G .
\end{aligned}
$$

As another example consider the case $a=f=g=0$ and

$$
h \equiv 1, \quad b(x, y) \equiv 1-e^{-\alpha}, \quad \alpha>0 .
$$

Then

$$
u^{\alpha}(t, x, y)=E_{x, y}^{R}\left\{e^{-\alpha N(t)}\right\}=\sum_{k=0}^{\infty} e^{-\alpha k} P_{x, y}^{R}\{N(t)=k\}
$$

which is the generating function of the distribution of the increasing process $N(t)$, satisfies the boundary value problem

t The collision operator $Q$ in (2.1.13) and (2.1.14) in still, however, a generator so that $Q 1=0$. This explains why, even with the terms $a$ and $b$, we continue to refer to the processes as a conservative transport processes. 


$$
\begin{aligned}
& \frac{\partial u^{\alpha}(t, x, y)}{\partial t}=\mathcal{L} u^{\alpha}(t, x, y), \quad t>0, \quad(x, y) \in G, \\
& u^{\alpha}(0, x, y)=0, \quad(x, y) \in G, \\
& \int_{S^{-}} B(x, y, d z) u^{\alpha}(t, x, z)-u^{\alpha}(t, x, y) \\
& \quad+\left(1-e^{-\alpha}\right) u^{\alpha}(t, x, y)+1=0, \quad t>0,(x, y) \in \partial G .
\end{aligned}
$$

\section{4. Asymptotic Problems and Homogenization}

Diffusion approximations are of principal interest to us here for both the absorbing and reflecting processes. After describing the diffusion limit we shall consider the homogenization problem.

Let $\varepsilon>0$ be a parameter and suppose that instead of $\mathcal{L}$ in (2.1.13) we define $\mathcal{L}^{\varepsilon}$ by

$$
\begin{aligned}
\mathcal{L}^{\varepsilon}= & \left(\frac{1}{\varepsilon} F^{(2)}(x, y)+F^{(3)}(x, y)\right) \cdot \frac{\partial}{\partial x} \\
& +\left(\frac{1}{\varepsilon^{2}} H^{(1)}(x, y)+\frac{1}{\varepsilon} H^{(2)}(x, y)+H^{(3)}(x, y)\right) \cdot \frac{\partial}{\partial y} \\
& +\frac{1}{\varepsilon^{2}} Q_{x} \\
= & \frac{1}{\varepsilon^{2}}\left(Q_{x}+H^{(1)}(x, y) \cdot \frac{\partial}{\partial y}\right) \\
& +\frac{1}{\varepsilon}\left(F^{(2)}(x, y) \cdot \frac{\partial}{\partial x}+H^{(2)}(x, y) \cdot \frac{\partial}{\partial y}\right) \\
& +\left(F^{(3)}(x, y) \cdot \frac{\partial}{\partial x}+H^{(3)}(x, y) \cdot \frac{\partial}{\partial y}\right)
\end{aligned}
$$

and denote the corresponding (free-space) process by $\left(X^{\varepsilon}(t), Y^{\varepsilon}(t)\right)$. We shall analyze the asymptotic limit of this process as $\varepsilon \rightarrow 0$. The way the various terms in $\mathcal{L}^{\varepsilon}$ are scaled relative to each other reflects (i) the situations of physical interest and (ii) the situations for which a nontrivial limit exists. Naturally, in addition to the usual hypotheses for the existence of the process for $\varepsilon>0$, we need hypotheses for the asymptotics. From (2.4.1) it is clear that the parameter $\varepsilon^{-1}$ is a measure of 
the frequency of jumps for the $Y^{\varepsilon}(t)$ process: We speed-up the jumps but at the same time increase the intensity of the external forces and the velocities.

Now the hypotheses for the asymptotics are, ${ }^{*}$ roughly, of two kinds

(i) Ergodic properties of

$$
I_{1}=Q_{x}+H^{(1)} \cdot \frac{\partial}{\partial y}
$$

(ii) Centering for $F^{(2)}$.

We also need a uniqueness theorem for the limiting diffusion process and smoothness if error estimates are desired. Only (i) presents a problem, generally, since (ii) will be simply imposed as a condition here.

The operator $\mathcal{L}_{1}$ is the generator of a Markov process on $S\left(=\boldsymbol{R}^{m}\right.$ up to now) with $x$ playing the role of a parameter. Let $Y_{x}(t), t \geq 0$ denote the process. Recall that $Q_{x}$ is defined by (2.1.14). We want. for the asymptotics, $Y_{x}(t)$ to be ergodic in a sufficiently strong sense so that the Fredholm alternative is valid for $\mathcal{L}_{1}$ in a convenient form. Physically this is a requirement about the local ( $x$ is fixed here) "velocity" process: that it equilibrate rapidly.

The centering condition is that $F^{(2)}$ averages to zero relative to the invariant measure of $Y_{x}(t)$.

So far we have discussed the free-space problem. What about the absorbing process of Section 2.1 with the scaled generator (2.4.1)? A certain amount of information can be deduced immediately from the result at hand on the free-space problem. However, this information relates to the limit of $X^{\varepsilon}(t)$. To find the limit properties of $Y^{\varepsilon}(t)$ when $X^{\varepsilon}$ is on $\partial \mathscr{D}$ it is necessary to do a boundary layer analysis. This turns out to be a problem of much the same form as (2.4.1) only now the ergodic theory for the problem corresponding to $\mathcal{L}_{1}$ is somewhat different.

The next three sections deal at length with various ergodic problems which will be encountered in Section 3. The reflected process also requires boundary layer considerations even though we restrict attention to $X^{\varepsilon}(t)$ and its limit.

Homogenization is the analysis of the asymptotic limit of processes

\footnotetext{
* They are discussed in detail in Section 3.
} 
with generators of the form (2.4.1) where, in addition, the vector functions $F^{(i)}, i=2,3, H^{(i)}, i=1,2,3$ and $q$ and $\pi$ of $Q$ change rapidly with $x$. Specifically, we assume that

$$
\begin{aligned}
F^{(i)} & =F^{(i)}(x, \zeta, y) \quad i=2,3, \\
H^{(i)} & =H H^{(i)}\left(x, \zeta, y^{\prime}\right) \quad i=1,2,3 \\
q & =q(x, \zeta, y) \\
\pi & =\pi\left(x, \zeta, y^{\prime}, A\right),
\end{aligned}
$$

where $\zeta \in R^{n}$ and as functions of $\zeta$ they are periodic of period 1 in all components and for all $x, y$. We may therefore consider the function as defined on the unit $n$-dimensional torus $T^{n}$. The operator $\mathcal{L}^{\varepsilon}$ is now defined as in (2.4.1) with the new $F, H, q, \pi$ and with $\zeta=\frac{x}{\varepsilon}$ i.e., with rapidly varying, periodically, coefficients.

Again a major portion of the asymptotic analysis of the homogenization problem is concerned with ergodic properties of the operator corresponding to $\mathcal{L}_{1}$ in $(2.4 .2)$ above. In the next section we analyze the situation that is needed for the free-space problem. Boundary layers and homogenization, simultaneously, can also be treated but we do not do so here. The analog of the results of Section 2. 7 with homogenization is valid again but will not be considered here.

\section{5. Ergodic Properties of Transport Operators}

It is necessary for the perturbation analysis to have available a certain amount of information about the ergodic properties of Markor processes on some state space $S$ with generators ${ }^{\dagger}$

$$
Q f(y)=q(y) \int_{s} \pi(y, d \approx) f(z)-q(y) f(y) .
$$

Here $q$ is a bounded measurable non-negative function and $\pi(y, A), y \in S$, $A \subset S$, is a measurable function of $y$ and a probability measure for each $y \in S$.

For the analysis of the homogenization problems it is necessary to

The continuity of $q$ and $\pi$ is removed here since the ergodic theory holds in greater generality. 
have available ergodic properties of Markov processes on $T \times S$, where $T$ is a finite dimensional torus, with generators

$$
\begin{aligned}
Q f(\zeta, y)= & F(\zeta, y) \cdot \frac{\partial f(\zeta, y)}{\partial \zeta} \\
& +q(\zeta, y) \int_{S} \pi(\zeta, y, d z) f(\zeta, z)-q(\zeta, y) f(\zeta, y) .
\end{aligned}
$$

Here $f(\zeta, y)$ is smooth in $\zeta, F(\zeta, y)$ is a smooth function of $\zeta$ with values in $\boldsymbol{R}^{n}$ where $n$ is the dimension of $T$ and $F \cdot \frac{\partial f}{\partial \zeta}$ stands for the inner product of $F$ with the gradient of $f$.

We introduce the following hypotheses regarding (2.5.1).

$$
0<q_{l} \leq q(y) \leq q_{u}<\infty
$$

for some constants $q_{l}$ and $q_{u}$.

(ii) There is a reference probability measure $\phi$ on $S$ such that $\pi(y, A)$ is absolutely continuous relative to $\phi$ with density $\pi(y, z)$ such that

$$
0<\pi_{l} \leq \pi(y, z) \leq \pi_{u}<\infty, \quad y, z \in S,
$$

where $\pi_{l}$ and $\pi_{u}$ are constants.

Let $P(t, y, A)$ denote the transition function corresponding to $Q$ of (2.5.1) and let $Y(t), t \geq 0$, be the corresponding process. We have that

$$
\begin{aligned}
P(t, y, A)=\chi_{A}(y) e^{-q(y) t} & \\
& +\int_{0}^{t} \int_{S} \pi(y, d z) P(t-s, z, A) q(y) e^{-q(y) s} d s
\end{aligned}
$$

and that the process is well defined in view of (2.5.3). It is well known that under hypotheses (i) and (ii) above there exists a unique invariant probability measure $\bar{P}(A)$, i.e.,

$$
\bar{P}(A)=\int_{S} \bar{P}(d z) P(t, z, A), \quad t \geq 0
$$

and that for $t$ large there is a constant $\alpha>0$ such that

$$
|P(t, y, A)-\bar{P}(A)| \leq e^{-a t}, \quad y \in S, A \subset S .
$$

As a consequence, the recurrent potential kernel $\psi(y, A)$

$$
\psi(y, A)=\int_{0}^{\infty}[P(t, y, A)-\bar{P}(A)] d t
$$


is well defined and the equation

$$
Q g(v)=-h(v), \quad v \in S
$$

has a bounded solution for cach bounded measurable $h$ such that

$$
\int_{s} h(y) \bar{P}(d y)=0 .
$$

In other word, the Fredholm alternative is valid for (2.5.9).

We are primarily interested in the corresponding results for $Q$ of (2.5.2). However, before continuing with the analysis of that problem we shall give, for completeness, the elementary arguments that yield the results (2.5.6), (2.5.7) (and hence the Fredholm alternative for $Q$ of (2. 5.1)).

First we show that there is an $h>0 \quad(h<\infty)$ and a $\delta>0$ such that

$$
P\left(h, y^{\prime}, A\right) \geq \delta \phi(A), \quad y \in S, A \subset S .
$$

From (2.5.5) it follows that

$$
P(t, y, A) \geq \int_{0}^{t} \int_{S} \pi(y, z) \chi_{A}(z) e^{-q(z)(t-s)} \phi(d z) q(y) e^{-q(y) s} d s .
$$

From this and (2.5.3), (2.5.4) we obtain

$$
P(t, y, A) \geq \pi_{l} q_{l} e^{-q_{u} t} t \phi(A),
$$

which implies (2.5.11) with $h=1 / q_{u}$, say, and $\delta=\pi_{l} q_{l}\left(q_{u} e\right)^{-1}$.

Next we verify that there is a positive constant $0<1$ such that for $n=2,3,4, \cdots$

(2. 5. 12) $|P(n h, y, A)-P(n h, z, A)| \leq \rho^{n-1}, \quad y, z \in S, A \subset S$.

Let $B_{y, z}^{+}$be the subset of $S$, depending on $y$ and $z$, where the signed measure $P(h, y, \cdot)-P(h, z, \cdot)$ is positive and $B_{y, z}^{-}$its complement (Hahn decomposition theorem). From

$$
\int[P(h, y, d \zeta)-P(h, \approx, d \zeta)]=0
$$

we conclude that

$$
\begin{aligned}
\int_{B_{y, z}^{+}}[P(h, y, d \zeta)-P(h, z, d \zeta)] \\
\quad=-\int_{B_{y, z}^{-}}[P(h, y, d \zeta)-P(h, z, d \zeta)]
\end{aligned}
$$


Moreover, there is a positive constant $\rho<1$ such that

$$
\int_{B_{y, z}^{+}}[P(h, y, d \zeta)-P(h, z, d \zeta)] \leq \rho<1
$$

In fact

$$
\rho=1-\hat{o}
$$

since

$$
\begin{aligned}
P\left(h, y, B^{-}\right)-P\left(h, z, B^{-}\right) & =1-\left[P\left(h, y^{\prime}, B^{-}\right)+P\left(h, \approx, B^{+}\right)\right] \\
& \leq 1-\delta\left[\phi\left(B^{-}\right)+\phi\left(B^{+}\right)\right] \\
& =1-\delta .
\end{aligned}
$$

Now we have for $n=2,3,4, \cdots$.

(2.5.16) $|P(n h, y, A)-P(n h, z, A)|$

$$
\begin{aligned}
& =\left|\int_{S}[P(h, y, d \zeta)-P(h, z, d \zeta)] P((n-1) h, \zeta, A)\right| \\
& =\mid \int_{B_{y, z}^{+}}[P(h, y, d \zeta)-P(h, z, d \zeta)] P((n-1) h, \zeta, A) \\
& \quad+\int_{B_{y, z}^{-}}[P(h, y, d \eta)-P(h, z, d \eta)] P((n-1) h, \eta, A) \mid \\
& \leq \int_{B_{y, z}^{+}}[P(h, y, d \zeta)-P(h, z, d \zeta)] \\
& \quad \times \sup _{\zeta, \eta}|P((n-1) h, \zeta, A)-P((n-1) h, \eta, A)| \\
& \leq 0 \sup _{\zeta, \eta}|P((n-1) h, \zeta, A)-P((n-1) h, \eta, A)|
\end{aligned}
$$

from which (2.5.12) follows by iteration. We are therefore in a position to ascertain the existence of a unique limit $\bar{P}(A)$ for $P(t, y, A)$ as $t \rightarrow \infty$ and that this satisfies (2.5.6). Clearly $\sup _{y} P(t, y, A)$ and $\inf _{y} P(t, y, A)$, with $A \subset S$ fixed, are, respectively, nonincreasing and nondecreasing with $t \uparrow \infty$ and

$$
\begin{aligned}
0 & \leq \lim _{t \uparrow \infty} \inf _{y} P(t, y, A) \leq \lim _{t \uparrow \infty} P(t, \zeta, A) \leq \varlimsup_{t \uparrow \infty} P(t, \zeta, A) \\
& \leq \lim _{t \uparrow \infty} \sup _{y} P(t, y, A) \leq 1 .
\end{aligned}
$$


But the right and left ends of this chain are identical by $(2.5 .12)$ so $\lim _{t \rightarrow \infty} P(t, y, A)$ exists and is independent of $y$; we call it $\bar{P}(A)$. The equation (2.5.6) follows from the Chapman-Kolmogorov equation and the bounded convergence theorem. Note that from (2.5.11) we have the lower bound

$$
\bar{P}(A) \geq \delta \not(A)
$$

To obtain the estimate (2.5.7) we write $t=n h+s$ ( $t$ is large), with $n \geq 2$ an integer and $0 \leq s<h$, and

$$
\begin{aligned}
\mid P(t, y, A) & -\bar{P}(A) \mid \\
= & \int[P(h+s, y, d \zeta)-\bar{P}(d \zeta)] P((n-1) h, \zeta, A) \mid .
\end{aligned}
$$

Now we decompose the right hand side as in (2.5.16) and deduce the result (2.5.7) from (2.5.12).

We note that once estimate $(2.5 .11)$ is obtained all the results follow (using also (2.5.3)). In view of this, the analysis of the process with (2.5.2) as generator, in particular the Fredholm alternative, will follow once an estimate like (2.5.11) is available. We shall proceed now with this objective.

Let us denote by $P(t, \zeta, y, A), A \subset T \times S, \zeta \in T, y \in S$ the transition function corresponding to $Q$ of (2.5.2). It satisfies the integral equation

$$
\begin{aligned}
& P(t, \zeta, y, A)=\chi_{A}(\xi(t), y) \exp \left(-\int_{0}^{t} q(\xi(s), y) d s\right) \\
& \quad+\int_{0}^{t} \int \pi(\hat{\xi}(s), y, z) P(t-s, \xi(s), z, A) q(\hat{\xi}(s), y) \\
& \quad \times \exp \left(-\int_{0}^{s} q(\tilde{\xi}(\gamma), y) d \gamma\right) \phi(d z) d s .
\end{aligned}
$$

Here we have assumed that $\xi(t)=\xi(t, \zeta, y) \in T$ satisfies the differential equations

$$
\frac{d \xi(t)}{d t}=F(\xi(t), y), \quad t>0, \xi(0, \zeta, y)=\zeta
$$

and $\pi(\zeta, y, B), B \subset S$, has density $\pi(\zeta, y, z)$ relative to a fixed probability measure $\phi(B)$ on $S$.

Evidently, it is necessary to impose restrictions on the nature of the 
solution curves of (2.5.19); in particular upon their dependence on $y \in S$. In the most interesting application in which (2.5.2) arises, homogenization in neutron transport problems, the space $S$ may be taken as the interior of the unit sphere in $n$ dimensions and $F(\zeta, y)=y$. We shall treat this case in detail and, to avoid lengthy expressions, we set $n=2$.

Thus, we shall assume that

$$
\begin{aligned}
& S=\left\{y \in \boldsymbol{R}^{2}:|y| \leq 1\right\} \\
& T=2 \text {-dimensional unit torus }
\end{aligned}
$$

and $(Z(t), Y(t))$ is the process on $T \times S$ with transition function $P(t, \zeta$, $y, D), D \subset T \times S$ satisfying

$$
\begin{aligned}
& P(t, \zeta, y, D)=\chi_{D}(\zeta+y t, y) \exp \left(-\int_{0}^{t} q(\zeta+y s, v) d s\right) \\
& \quad+\int_{0}^{t} \int \pi\left(\zeta+y s, y, y_{1}\right) P\left(t-s, \zeta+y s, y_{1}, D\right) q(\zeta+y s, y) \\
& \quad \times \exp \left(-\int_{0}^{s} q(\zeta+y \gamma, y) d \gamma\right) d y_{1} d s .
\end{aligned}
$$

Here we have assumed that the jump probabilities $\pi$ are absolutely continuous with respect to Lebesgue measure which we denote by $d y$; it is normalized to total mass one on $S$. Lebesgue measure on $T$, normalized again, is denoted by $d \zeta$. We shall show that there is an $h>0 \quad(h<\infty)$ and a $\delta>0$ such that for any $D \subset T \times S$

$$
P(h, \zeta, y, D) \geq \delta \int_{D} \int d y d z
$$

This is just like (2.5.11) so the results (2.5.6)-(2.5.10) (the Fredholm alternative) will follow for the present problem also.

It is enough to show that $(2.5 .22)$ holds for sets $D$ of the form $A \times B$ where $A \subset T$ and $B \subset S$.

We iterate (2.5.21) three times, keep the third term and discard the others and use (2.5.3) and (2.5.4) to obtain the lower bound

$$
\begin{aligned}
& P(t, \zeta, y, A \times B) \geq\left(\pi_{l} q_{l}\right)^{2} e^{-q_{u} t} \\
& \quad \cdot \int_{0}^{t} d s_{1} \int_{0}^{t-s_{1}} d s_{2} \int_{S} d y_{1} \int_{S} d y_{2} \chi_{A \times B}\left(\zeta+y s_{1}+y_{1} s_{2}+y_{2}\left(t-s_{1}-s_{2}\right), y_{2}\right) .
\end{aligned}
$$

By restricting the ranges of $s_{1}$ and $s_{2}$ we obtain further 
(2.5.23) $\quad P(t, \zeta, y, A \times B) \geq\left(\pi_{l} q_{l}\right)^{2} e^{-q_{u} t}$

$$
\cdot \int_{0}^{t / 3} d s_{1} \int_{t / 3}^{2 t / 3} d s_{2} \int_{B} d y_{2} \int_{S} d y_{1} \chi_{A}\left(\zeta+\nu s_{1}+y_{1} s_{2}+y_{2}\left(t-s_{1}-s_{2}\right)\right) .
$$

In (2.5.23) the point $\zeta+y s_{1}+y_{1} s_{2}+y_{2}\left(t-s_{1}-s_{2}\right) \in \mathbb{R}^{2}$ is identified with its corresponding base point in $T=[0,1) \times[0,1)$. If we introduce the set

$$
\widetilde{A}=\bigcup_{n \in \mathbb{Z}^{3}}\{A+n\} \subset \boldsymbol{R}^{2},
$$

then we can replace $A$ by $\tilde{A}$ in $(2.5 .23)$ and allow the argument of $\chi_{\tilde{A}}$ to be in $\boldsymbol{R}^{2}$.

Let $\zeta^{\prime}=\zeta+y s_{1}+y_{2}\left(t-s_{1}-s_{2}\right)$. We have

$$
\begin{aligned}
\int_{S} d y_{1} \chi_{A}\left(\zeta^{\prime}+y_{1} s_{2}\right) & =\int_{S} d y_{1} \chi_{\tilde{A}}\left(\zeta^{\prime}+y_{1} s_{2}\right) \\
& =\int_{S} d y_{1} \sum_{n \in \mathbb{Z}^{2}} \chi_{A}\left(\zeta^{\prime}-n+y_{1} s_{2}\right) .
\end{aligned}
$$

In this equality, the point $\zeta^{\prime}+y_{1} s_{2}$ is regarded as a point in $T$ (reduced point), with $A \subset T$, on the left. On the right, $\zeta^{\prime}+y_{1} s_{2}$ is regarded as a point in $\boldsymbol{R}^{2}$ and $A \subset[0,1) \times[0,1) \subset \mathbb{R}^{2}$. There is a point $n^{\prime}$ of the lattice $\mathbb{Z}^{2}$ such that $\left|\zeta^{\prime}-n^{\prime}\right|<1$. We have then the lower bound

$$
\int_{S} d y_{1} \chi_{\tilde{A}}\left(\zeta^{\prime}+y_{1} s_{2}\right) \geq \int_{S} d y_{1} \chi_{A}\left(\zeta^{\prime}-n^{\prime}+y_{1} s_{2}\right) \geq \frac{1}{s_{2}^{2}} \int_{S} \chi_{A}(z) d z
$$

provided $s_{2}>3$. Combining this with $(2.5 .24)$ we have that

$$
\int_{S} d y_{1} \chi_{A}\left(\zeta^{\prime}+y_{1} s_{2}\right) \geq \frac{1}{s_{2}^{2}} \int_{A} d \approx,
$$

provided $s_{2}>3$.

If $t>9$ in $(2.5 .23)$ then $s_{2}>3$ and (2.5.25) applies. Thus,

$$
P(t, \zeta, y, A \times B) \geq\left(\pi_{l} q_{l}\right)^{2} e^{-q_{u} t} \int_{A \times B} d z d y
$$

which proves (2.5.22) (this argument is an improvement of a previous one, supplied to us by the referee).

For a general orbit structure obtained by solving (2.5.19) one way one might derive the result (2.5.22) is by assuming that each coordinate of $\xi(t)$ is bounded above and below by constant multiples of the orbit of some simpler problem such as the rectilinear one just analyzed. 
We summarize the results concerning (2.5.2) as follows:

Theorem. Let $S=\left\{y \in \boldsymbol{R}^{n}:|y| \leq 1\right\}, T=n$-dimensional torus and for $(\zeta, y) \in T \times S$ and $f(\zeta, y)$ a real bounded and differentiable in $\zeta$ function define

$$
\begin{aligned}
Q f(\zeta, y)=y \cdot \frac{\partial f(\zeta, y)}{\partial \zeta} & +q(\zeta, y) \int_{s} \pi\left(\zeta, y, y^{\prime}\right) f\left(\zeta, y^{\prime}\right) d y^{\prime} \\
& -q(\zeta, y) f(\zeta, y) .
\end{aligned}
$$

Assume that (2.5.3) and (2.5.4) hold for $q$ and $\pi$ (as functions of the extra variable's as well). Then $Q$ is the generator of an ergodic Markov process $(Z(t), Y(t))$ on $T \times S$ reith a unique invariant measure $\bar{P}(d \zeta, d y)$. Furthermore, if $h\left(\zeta, y^{\prime}\right)$ is a bounded function on $T \times S$ such that

$$
\int_{T \times S} h(\zeta, y) \bar{P}(d \zeta, d y)=0
$$

the equation

$$
Q g(\%, y)=-h(\zeta, y)
$$

has a bounded solution, i.e., the Fredholm alternative holds.

Remark. In the theorem the equation $-Q g=h$ is understood to hold in its integral form (cf. (2.5.21)). Thus $h$ need not be differentiable in $\zeta$ and so $g$ need not be differentiable in $\zeta$.

If $q, \pi$ and $h$ are differentiable in $\zeta$, then so is $g$ and $Q g=-h$ holds in the usual way.

If $q, \pi$ and $h$ depend differentiably on a parameter $x$, then $g$ also depends differentiably on $x$.

\subsection{Reflection and Transmission Operators for Half-Space Problems}

The boundary layer analysis, just as the homogenization problem, requires information about the ergodic properties of certain processes defined on a half-line. In this and the next section we shall examine in 
detail these properties. We begin by formulating the problem under consideration.

Let $S$ be a compact metric space (such as the unit sphere in $\mathbb{R}^{n}$ ), the state space of a Markov process $Y(t)$, let $\phi$ be a fixed reference probability measure on it and let $Q$, defined by

$$
Q f(y)=q(y) \int_{S} \pi\left(y, y^{\prime}\right) f\left(y^{\prime}\right) \phi\left(d y^{\prime}\right)-q(y) f(y)
$$

be the generator of $Y(t), t \geq 0$. We assume that

$$
\begin{aligned}
& 0<q_{l} \leq q(y) \leq q_{u}<\infty, \\
& 0<\pi_{l} \leq \pi\left(y, v^{\prime}\right) \leq \pi_{u}<\infty,
\end{aligned}
$$

where $q_{l}, q_{u}, \pi_{l}$ and $\pi_{u}$ are constants. As discussed in the previous section, $Y(t)$ is ergodic with invariant measure $\bar{P}(A)$ and if $P(t, y, A)$, $A \subset S$, is the transition function of $Y^{Y}(t)$,

$$
P(t, y, A)=P\{Y(t) \in A \mid Y(0)=y\},
$$

then, there is an $\alpha>0$ such that

$$
\sup _{y \subseteq S} \sup _{A \subset S}|P(t, y, A)-\bar{P}(A)| \leq e^{-\alpha t}
$$

at least for $/$ large enough. With $q(y)$ and $\pi\left(y^{\prime}, y^{\prime}\right)$ continuous, $Y(t)$ is a right-continuous strong Markov process on $S$.

Let $z(y)$ be a function from $S$ into $[-1,1]$, say, which is continuous, and assume that

$$
\int_{s} \approx(y) \bar{P}(d y)-0
$$

We also assume that $\approx\left(y^{\prime}\right)$ is nontrivial i.e.,

$$
\bar{P}\{\approx(y) \neq 0\}>0 \text {. }
$$

For the potential theory of the half-space problem (Theorem 2, Section $7)$, it will be necessary to introduce one more condition regarding $\approx(y)$ as follows. Let $\approx(Y(t)) \equiv \approx t$ be the stationary process on $-\infty<t<\infty$ with values in $[-1,1]$ obtained by letting $Y(t)$ be as above and with initial distribution $\bar{P}$ i.e. $P\{Y(0) \in A\}=\bar{P}(A)$. By (2.6.6) $\quad E\left\{z_{t}\right\}=0$ and 


$$
E\left\{z_{s} z_{s+t}\right\}=\iint P\left(t, y_{1}, d y_{2}\right) \bar{P}\left(d y_{1}\right) z\left(y_{1}\right) z\left(y_{2}\right) \equiv \Gamma(t), \quad t \geq 0,
$$

is the covariance function. Note that $\Gamma(t)$ is even about $t=0$. Moreover, in view of (2.6.5),

$$
\begin{aligned}
& \left(2.6 .6^{\prime \prime}\right) \quad \sigma^{2}=\int_{-\infty}^{\infty} \Gamma(t) d t=\left.\int_{-\infty}^{\infty} e^{i \omega t} \Gamma(t) d t\right|_{\omega=0} \\
& =2 \iiint_{0}^{\infty}\left[P\left(t, y_{1}, d y_{2}\right)-\bar{P}\left(d y_{2}\right)\right] d t \bar{P}\left(d y_{1}\right) z\left(y_{2}\right) z\left(y_{1}\right) \\
& =-2 \int \bar{P}\left(d y_{2}\right) z\left(y_{2}\right) Q^{-1} z\left(y_{2}\right) \\
& =\lim _{t \uparrow \infty} E\left\{\left(\frac{1}{\sqrt{t}} \int_{0}^{t} z_{s} d s\right)^{2}\right\} \geq 0 \text {. }
\end{aligned}
$$

In the sequel we assume that $\sigma^{2}>0$ as the notation indicates.

On $(-\infty, \infty) \times S$ we consider a Markov process, which we must show is well defined, with infinitesimal generator

$$
\begin{gathered}
\widetilde{Q} f(\eta, y)=z(y) \frac{\partial f(\eta, y)}{\partial \eta}+Q f(\eta, y), \\
y \in S,-\infty<\eta<\infty,
\end{gathered}
$$

where $Q$ acts on $f$ as a function of $y$ only and it is given by (2.6.1). Let $Y(t), t \geq 0$ be the process on $S$ generated by $Q$. Define $H(t)$, $t \geq 0$ by

$$
H(t)=\eta+\int_{0}^{t} z(Y(s)) d s, \quad \eta \in(-\infty, \infty) .
$$

Since only finitely many jumps occur, for $Y$, in finite time intervals and since $|z| \leq 1$ it follows that $H(t)$ is well defined and continuous and (2.6.7) is the generator of $(H(t), Y(t)), t \geq 0$.

We decompose the state space $S$ into sets as follows.

$$
\begin{aligned}
& S^{-}=\{y \in S: \approx(y) \geq 0\} \\
& S^{-}=\{y \in S: \approx(y)<0\}
\end{aligned}
$$

Let $\tau$ be the first time that $H(t)=0$ starting from $\eta<0$ and with $Y(0)$ $=y \in S$, which is a stopping time. If $\eta=H(0)=0$ and $y \in S^{\perp}$ we define $:=0$ but if $y \in S^{-}$we allow the process tn evolve until the time $\tau$ that 
again $H(\tau)=0$. Clearly, from (2.6.8) we see that $Y(\tau) \in S$ with this definition. We must also show that $\tau<\infty$ with probability one.

Because of (2.6.6) and the validity of the Fredholm alternative for $Q, Q^{-1} \approx$ exists and is a bounded function of $y$. Thus

(2.6.10) $\quad f(\eta, y)=\eta-\left(Q^{-1} \approx\right)(y) \quad \eta \in(-\infty, \infty), y \in S$,

satisfies $\widetilde{Q} f=0$ i.e., is a harmonic function. Let $\eta \in(a, b)$, a finite interval, and let $\tau_{a b}$ be the first time $H(t)$ is equal to $a$ or $b$ starting from $\eta$ with $Y(0)=y$. From the optional stopping theorem we have

$$
E_{\eta, y}\left\{f\left(H\left(\tau_{a b}\right), Y\left(\tau_{a b}\right)\right)\right\}=f(\eta, v) .
$$

Hence, if

$$
A=\{a \text { is reached before } b\}
$$

and $\bar{A}$ is the complement of $\bar{A}$, we have

$$
\begin{aligned}
E_{\eta, y}\{ & \left.f\left(a, Y\left(\tau_{a b}\right)\right) \mid A\right\} P_{\eta, y}\{A\} \\
& +E_{\eta, y}\left\{f\left(b, Y\left(\tau_{a b}\right)\right) \mid \bar{A}\right\} P_{\eta, y}\{A\}=f\left(\eta, y^{\prime}\right) .
\end{aligned}
$$

Letting $a \rightarrow-\infty$ and noting that $f(a, y) \rightarrow-\infty$ for all $y$ we conclude that for any $\eta<b$ and $y \in S$.

$$
P_{n, y}\{b \text { is reached }\}=1
$$

Therefore, the random variable $Y(\tau) \in S$ above is well defined for any $\eta<0$ and $y \in S$ or $\eta=0$ and $y \in S^{-}$.

Let

$$
U(\eta, y, A)=E_{\eta, y}\{Y(\%) \in A\}
$$

where,

$$
(\eta, y) \in\{\eta<0, y \in S\} \cup\left\{\eta=0, y \in S^{-}\right\}
$$

and

$$
A \subset S^{+}
$$

We have just shown that (2.6.11) is vell defined and by the usual arguments of Sections 2.1 and 2.2 we have that $U$ satisfies the boundary value problem ${ }^{+}$

\footnotetext{
'Its integral equation form. This is the convention throughout.
} 
(2.6.12)

$$
\begin{gathered}
z(y) \frac{\partial U}{\partial \eta}+Q U=0, \quad\{\eta<0, y \in S\} \cup\left\{\eta=0, y \in S^{-}\right\}, \\
U(0, y, A)=\chi_{A}(y), \quad y \in S^{+}, A \subset S^{+} .
\end{gathered}
$$

Let $f(y)$ be a bounded measurable function on $S^{+}$and let for $\eta \leq 0$

$$
\begin{aligned}
\left(T_{\eta} f\right)(y) & =\int_{S^{+}} U\left(\eta, y, d y^{\prime}\right) f\left(y^{\prime}\right) \\
& =E_{\eta, y}\{f(Y(\tau))\}, \quad y \in S^{+} .
\end{aligned}
$$

The linear operators $T_{\eta}, \eta \leq 0$, are called the transmission operators. They are transition operators of a Markov process induced on $S^{\tau}$. From the strong Markov property of $(H(t), Y(t))$ and the translation invariance of the generator $\widetilde{Q}$ (translation in $\eta$ ) we deduce that for $\eta_{1}, \eta_{2} \leq 0$

$$
\left(T_{\eta_{1}+\eta_{2}} f\right)(y)=\left(T_{\eta_{1}}\left(T_{\eta_{2}}\right)\right)(y)=\left(T_{\eta_{2}}\left(T_{\eta_{1}} f\right)\right)(y), \quad y \in S^{+} .
$$

The principal result of the next section is that as $\eta \rightarrow-\infty T_{n} f$ approaches a limit exponentially fast (Theorem 1). This means that the induced process on $S^{+}$is strongly ergodic. It will be shown in Section 2. 7 that there is a probability measure $\bar{U}(A), A \subset S^{-}$and a constant $\alpha>0$ such that for all $-\eta$ sufficiently large

$$
|U(\eta, y, A)-\bar{U}(A)| \leq e^{\alpha \eta},
$$

for all $A \subset S^{+}$and $y \in S^{+}$. If $f(y)$ is bounded and measurable on $S^{+}$and if

$$
\bar{f}=\int_{S^{+}} f(y) \bar{U}(d y),
$$

then (2.6.14) implies that

$$
\lim _{\eta \rightarrow-\infty} T_{\eta} f(y)=\bar{f}
$$

uniformly in $y \in S^{\perp}$.

The reflection operator $R$ is defined by

(2.6.17) $R f(y)=\int_{S^{+}} U\left(0, y, d y^{\prime}\right) f\left(y^{\prime}\right)=E_{0, y}\{f(Y(\tau))\}, \quad y \in S^{-}$.

The terminology for both $R$ and $T_{\eta}$ is suggestive of the meaning of these objects in the moving particle model of a transport process.

There is a useful relationship between the limit $\bar{f}$ in (2.6.16) (or 
2.6.15) and $R f$ which we shall now derive.

For $f\left(\eta, y^{\prime}\right)$, a differentiable function of $\eta$ and a bounded measurable function of $y, \widetilde{Q} f(\eta, y)$ is defined by (2.6.7). We now define $\widetilde{Q}^{*}$ acting on $f^{*}(\eta, A), \eta \leq 0, A \subset S$, which are differentiable for each fixed $A$ and measures for each fixed $\eta$ as follows:

$$
\begin{aligned}
& \widetilde{Q}^{*} f^{*}(\eta, A)=-\int_{A} z(y) \frac{\partial f^{*}}{\partial \eta}(\eta, d y) \\
& \quad+\int_{S} f^{*}(\eta, d y) q(y) \int_{A} \pi\left(y, y^{\prime}\right) \phi\left(d y^{\prime}\right)-\int_{A} q(y) f^{*}(\eta, d y) .
\end{aligned}
$$

With this notation we have the following Green's identity: for any $\eta_{1} \leq \eta_{2}$ and any $f(\eta, y)$ such that $\widetilde{Q} f=0$ and $f^{*}(y, A)$ such that $\widetilde{Q}^{*} f^{*}=0$

$$
\begin{aligned}
0 & =\int_{\eta_{1}}^{\eta_{2}} d \eta \int_{s}\left[f^{*}(\eta, d y) \widetilde{Q} f(\eta, y)-f(\eta, y) \widetilde{Q}^{*} f^{*}(\eta, d y)\right] \\
& =\int_{s} z(y) f\left(\eta_{2}, y\right) f^{*}\left(\eta_{2}, d y\right)-\int_{s} z(y) f\left(\eta_{1}, y\right) f^{*}\left(\eta_{1}, d y\right) .
\end{aligned}
$$

Let $h(y)$ be a bounded measurable function on $S^{\tau}$ and let $u(\eta, y)$ be the solution of

$$
\begin{gathered}
z(y) \frac{\partial u}{\partial \eta}+Q u=0, \quad\{\eta<0, y \in S\} \cup\left\{\eta=0, y \in S^{-}\right\} \\
u(0, y)=h(y), \quad y \in S^{+} .
\end{gathered}
$$

Let $\bar{h}=\lim _{\eta \rightarrow-\infty} u(\eta, y)$ which is a constant by (2.6.16). We apply first the jdentity (2.6.19) with $\eta_{2}=0, \eta_{1}=-\infty$ to the functions

$$
\begin{aligned}
& f(\eta, y)=u(\eta, y)-\bar{h} \\
& f^{*}(\eta, A)=\bar{P}(A) .
\end{aligned}
$$

This yields the result

$$
\int_{s} z(y)(u(0, y)-\bar{h}) \bar{P}(d y)=0
$$

which we may rewrite, using (2.6.17), as

$$
\int_{S^{+}} z(y) h(y) \bar{P}(d y)+\int_{s^{-}} z(y) R h(y) \bar{P}(d y)=0 .
$$

Let us apply (2.6.19) with $\eta_{2}=0, \eta_{1}=-\infty$ to the functions

$$
f(\eta, y)=u(\eta, y)-\bar{h}
$$




$$
f^{*}(\eta, A)=\eta \bar{P}(A)-\int_{S} z(y) \bar{P}(d y) \psi(y, A) .
$$

Here $\psi(y, A)$ is the recurrent potential kernel of $Y(t)$ defined by (2.5.8) where $P(t, y, A)$ is the transition function of $Y(t)$. Clearly this choice of $f^{*}$ satisfies $\widetilde{Q}^{*} f^{*}=0$ so the identity (2.6.19) applies. We obtain

$$
\text { (2. 6. 23) } \quad \int_{s} z(y)(u(0, y)-\bar{h}) \int_{s} z\left(y^{\prime}\right) \bar{P}\left(d y^{\prime}\right) \psi\left(y^{\prime}, d y\right)=0 \text {. }
$$

This implies that

$$
\bar{h}=\frac{\int_{S} z(y) u(0, y) \int_{S} z\left(y^{\prime}\right) \bar{P}\left(d y^{\prime}\right) \psi\left(y^{\prime}, d y\right)}{\int_{S} z(y) \int_{S} z\left(y^{\prime}\right) \bar{P}\left(d y^{\prime}\right) \psi\left(y^{\prime}, d y\right)}
$$

which, using (2.6.17) and rearranging, takes the form

$$
\bar{h}=\frac{\int_{S} z\left(y^{\prime}\right) \bar{P}\left(d y^{\prime}\right)\left[\int_{S^{+}} h(y) z(y) \psi\left(y^{\prime}, d y\right)+\int_{S^{-}} R h(y) z(y) \psi\left(y^{\prime}, d y\right)\right]}{\int_{S} z\left(y^{\prime}\right) \bar{P}\left(d y^{\prime}\right) \int_{S} z(y) \psi\left(y^{\prime}, d y\right)}
$$

with the denominator positive by the hypothesis below (2.6.6" $6^{\prime \prime}$. This is the desired relationship between $\bar{h}$ and $R h$.

We turn next to the half-space problem with reflection. The reflected process $\left(H^{R}(t), Y^{R}(t)\right)$ is defined on the state space $\{(-\infty, 0] \times S\}$. In the interior set of points $\{(-\infty, 0) \times S\} \cup\left\{\{0\} \times S^{-}\right\}$, the generator is $\widetilde{Q}$, given by (2.6.7), as before. On the boundary $\{0\} \times S^{+}$the process is reflected instantaneously according to a given probability law $B(y, A)$, $y \in S^{+}, A \subset S^{-}$, just as in Section 2.2. We assume that the kernel $B$ satisfies condition (2.2.9) ( $x$ corresponds to the single point $\eta=0$ here) and this insures that the process hits the boundary finitely often in finite time intervals, with probability one. We denote by $P_{\eta, y}^{R}$ and by $E_{\eta, y}^{R}$ the probability distribution and expectation starting at $(\eta, y)$ of the reflected process.

We consider the solution of the following initial-boundary value prob$\operatorname{lem}^{\dagger}$

\footnotetext{
${ }^{\dagger}$ Recall the convention that (2.6.26) is taken in its integral equation form.
} 
$(2.6 .26)$

$$
\begin{gathered}
\frac{\partial u(t, \eta, y)}{\partial t}=z(y) \frac{\partial u(t, \eta, y)}{\partial \eta}+Q u, \\
\{\eta<0, y \in S\} \cup\left\{\eta=0, y \in S^{-}\right\}, t>0, \\
u(0, \eta, y)=0 \\
\int_{S^{-}} B\left(y, d y^{\prime}\right) u\left(t, 0, y^{\prime}\right)-u(t, 0, y)=-h(y), \quad y \in S^{+} .
\end{gathered}
$$

From (2.3.5) we obtain the representation

$$
\begin{aligned}
u(t, \eta, y) & =E_{\eta, y}^{R}\left\{\int_{0}^{t} h\left(Y^{R}(s-)\right) d N(s)\right\} \\
& =E_{\eta, y}^{R}\left\{\sum_{k=1}^{\infty} h\left(Y^{R}\left(\sigma_{k}-\right)\right) \chi\left(\sigma_{k} \leq t\right)\right\}
\end{aligned}
$$

where $N(t)$ and $\sigma_{0}, \sigma_{1}, \sigma_{2}, \cdots$ are defined in (2.2.21).

It is clear that in order to treat the time homogeneous problem, i.e., the problem obtained by setting $\frac{\partial u}{\partial t}=0$ on the left side in equation (2.6.26), it is necessary to impose restrictions on $h$ or else a solution will not exist. When a solution does exist it will not be unique without further specifications, for example behavior for $\eta \rightarrow-\infty$. Thus we need a Fredholm alternative for the problem

$$
\begin{aligned}
& z(y) \frac{\partial u(\eta, y)}{\partial \eta}+Q u(\eta, y)=0, \\
& \{\eta<0, y \in S\} \cup\left\{\eta=0, y \in S^{-}\right\}
\end{aligned}
$$

$$
\int_{S^{-}} B\left(y, d y^{\prime}\right) u\left(0, y^{\prime}\right)-u(0, y)=-h(y), \quad y \in S^{+}
$$

and for uniqueness we impose the additional condition

$$
u(\eta, y) \rightarrow 0 \quad \text { as } \quad \eta \rightarrow-\infty .
$$

In order to see more clearly what the restriction on $h$ must be we employ the reflection operator $R$ of $(2.6 .17)$ to write $\left(2.6 .28^{\prime}\right)$ as follows.

$$
B R u(0, y)-u(0, y)=-h(y), \quad y \in S^{+} .
$$

Here $B$ is the operator with kernel $B\left(y^{\prime}, A\right)$ as in $\left(2.6 .28^{\prime}\right)$. If the operator $I-B R$ was invertible then problem (2.6.28) would reduce to 
problem (2.6.20) with $h$ replaced by $(I-B R)^{-1} h$. However, the operator $B R$ has 1 as an eigenvalue since both $R$ and $B$ transform the function one (on their respective ranges) to itself. Equivalently, from (2. 6. 27)

$$
\lim _{t \rightarrow \infty} E_{\eta, y}^{R}\left\{\sum_{k=1}^{\infty} h\left(Y^{R}\left(\sigma_{k}-\right)\right) \chi\left(\sigma_{k} \leq t\right)\right\}
$$

will not exist $(N(t) \rightarrow \infty$ with probability one) unless $h$ is appropriately restricted.

The operator $B R$ is the transition operator of a discrete-time Markov process on $S^{+}$and for $f\left(y^{\prime}\right)$ bounded measurable on $S^{-}$we have

$$
\begin{aligned}
B R f(y) & =\int_{S^{-}} B\left(y, d y^{\prime}\right) \int_{S^{+}} U\left(0, y^{\prime}, d y^{\prime \prime}\right) f\left(y^{\prime \prime}\right) \\
& =\int_{S^{+}} f\left(y^{\prime \prime}\right) \int_{S^{-}} B\left(y, d y^{\prime}\right) U\left(0, y^{\prime}, d y^{\prime \prime}\right) .
\end{aligned}
$$

If we assume, as we will do, that there exists a probability measure ip on $S^{-}$and a $\delta>0$ such that

$$
B(y, A) \geq \delta \phi(A),
$$

then it follows that $B R$ is strongly ergodic with $\bar{U}_{R}(A), A \subset S^{\perp}$, its unique invariant measure (and the Fredholm alternative holds). It is enough to notice that

$$
\begin{gathered}
\left|B R\left(y_{1}^{\prime}, A\right)-B R\left(y_{2}, A\right)\right| \leq \rho<1, \\
y_{1}, y_{2} \in S^{-}, A \subset S^{+},
\end{gathered}
$$

as a consequence of (2.6.31). The rest goes as in Section 2.5. In fact

$$
\begin{aligned}
\mid B R & \left(y_{1}, A\right)-B R\left(y_{2}, A\right) \mid \\
& =\left|\int_{S^{-}}\left[B\left(y_{1}, d y\right)-B\left(y_{2}, d y\right)\right] U(0, y, A)\right| \\
& \leq \int_{S_{+,}^{-} y_{1}, y_{2}}\left[B\left(y_{1}, d y\right)-B\left(y_{2}, d y\right)\right]=\rho<1
\end{aligned}
$$

where $S_{+, y_{1}, y_{2}}^{-}$is the set of $y$ 's where $B\left(y_{1}, A\right)-B\left(y_{2}, A\right)$ is positive (Hahn decomposition). I Tere $\rho=1--\delta$ by (2.6.31) as in (2.5.14) .

Thus, the relevant ergodic properties for the reflected process can be cleduced in an elementary way from a hypothesis such as (2.6.31). 
We require no ergodic or other properties from $U$; all is based on the $B$. If we know that $U(0, y, A), y \in S^{-}, A \subset S^{\perp}$, satisfies a lower bound like the one for $B$, then that in turn suffices for the ergodicity of $B R$ without assumptions on $B$, other than the ones made before (2.6.30). Note that the invariant measure $\bar{U}_{R}$ of $B R$ is the asymptotic distribution of $Y^{R}\left(\sigma_{k}-\right)$ as $k \rightarrow \infty$.

Let us return to $(2.6 .28)$ and assume that

$$
\int_{S^{+}} \bar{U}_{R}(d y) h(y)=0 \text {. }
$$

Then, by the Fredholm alternative and (2.6.32),

$$
u(0, y)=\sum_{k=0}^{\infty}\left((B R)^{k}-\bar{U}_{R}\right) h(y)+c, \quad y \in S^{+},
$$

where $(B R)^{0}(y, A)=\chi_{A}(y)$ and $(B R)^{k}$ for $k>1$ are the interates of the transition operator $B R$. The sum in (2.6.33) converges since $(B R)^{k}$ tends to $\bar{U}_{R}$ geometrically fast as $k \rightarrow \infty$. In (2.6.33) we have also added a constant $c$ on the right hand side which is to be determined from (2.6.29).

In fact, with $u(0, y), y \in S^{+}$, determined, problem (2.6.28) is identical to (2.6.20). Thus for each constant $c,(2.6 .28)$ and (2.6.28') have a unique solution $u(\eta, v ; c)$. From the ergodic properties of $U(\eta, v, A)$ as $\eta \rightarrow-\infty$ (c.f. $(2.6 .14)$ ) it follows that $\lim _{\eta \rightarrow-\infty} u\left(\eta, y^{\prime} ; c\right)$ will exist for each $c$ and we will have

(2. 6. 34) $\lim _{\eta \rightarrow-\infty} u(\eta, y ; c)=\int_{S^{+}} \bar{U}(d y) \sum_{k=0}^{\infty}\left((B R)^{k}-\bar{U}_{R}\right) h(y)+c$.

If we choose the constant $c$ so that the right-hand side of (2.6.34) is zero, then (2.6.29) is satisfied and (2.6.28)-(2.6.29) indeed has a unique solution if $h$ is such that (2.6.32) holds. The special constant $c$ so chosen will be denoted $c_{h}$

$$
c_{h}=-\int_{S^{+}} \bar{U}(d y) \sum_{k=0}^{\infty}\left((B R)^{k}-\bar{U}_{R}\right) h(y) .
$$

The solvability condition on $h$ for the problem

$$
\approx(y) \frac{\partial u}{\partial \eta}+Q u=-f(\eta, y), \quad \eta<0,
$$




$$
\begin{gathered}
u \rightarrow 0 \text { exponentially fast as } \eta \rightarrow-\infty, \\
\int_{S^{-}} B u-u=-h, \quad \eta=0, y \in S^{+},
\end{gathered}
$$

with $f \rightarrow 0$ exponentially fast as $\eta \rightarrow-\infty$, is the same as above, namely (2.6.32). However, the constant $c$ must be chosen in accordance with Theorem 2 of Section 2.7 (cf. (2.7.41)) and it depends on $h$ and $f$.

\section{7. Potential Theory for the Half-Space Problem}

In this section we shall first show that, as $\eta \rightarrow-\infty, U(\eta, y, A)$ has a limit $\bar{U}(A)$ and that (2.6.14) holds. Then we shall prove some related results that will be also needed in the asymptotic analysis of the following sections.

Theorem $1^{\dagger}$ Let $U(\eta, y, A), \eta \leq 0, y \in S, A \subset S^{-}$be the solution of (2.6.12). Then there is an $\eta_{0},-\infty<\eta_{0}<0$ and $a \rho<1$ such that

$$
\left|U\left(\eta_{0}, \searrow_{1}^{\prime}, A\right)-U^{\top}\left(\eta_{0}, y_{2}, A\right)\right| \leq \rho, \quad y_{1}, y_{2} \in S, A \subset S^{+} .
$$

Remark. From (2.7.1) it follows that

$$
\lim _{\eta \rightarrow-\infty} U(\eta, y, A)=\bar{U}(A), \quad A \subset S^{+}
$$

exists uniformly in $y \in S$ and, moreover, the limit is approached exponentially fast i.e., (2.6.14) holds. The passage from (2.7.1) to these results is as in Section 2. 5 .

Proof. Let $\widetilde{P}(t, \eta, y, D)$ be the transition function of $(H(t), Y(t))$ whose generator is $\widetilde{Q}$ of (2.6.7). Here $D \subset\{(-\infty, 0] \times S\}$. Let $t_{0}$ be a positive number to be chosen later and suppose that $t_{0}<-\eta_{0}$. Let $P(t, y, A), y \in S, A \subset S$, be the transition function corresponding to the generator $Q$ of (2.6.1) i.e.,

$$
P(t, y, A)=P\{Y(t) \in A \mid Y(0)=y\},
$$

and let $\bar{P}(A), A \subset S$, be its invariant measure. From the results of

\footnotetext{
t This theorem is due to S. R. S. Varadhan (private communication).
} 
Section 2.5 we know that (2.6.5) holds. Since $z(y)$ in $(2.6 .7)$ is not identically zero and since (2.6.6) holds, it follows that for some $\theta>0$

$$
\bar{P}\{|z(y)| \geq \theta\}=r>0 .
$$

Let us also choose $t_{0}$ sufficiently large so that

$$
\begin{aligned}
& \left|P\left(t_{0}, y, A\right)-\bar{P}(A)\right| \leq \frac{\gamma}{4}, \\
& \left|P\left(t_{0}, y_{1}, A\right)-P\left(t_{0}, y_{2}, A\right)\right| \leq \frac{\gamma}{2},
\end{aligned}
$$

as we know is possible by (2.6.5).

We shall need the following general fact about two probability measures $P_{1}$ and $P_{2}$ (on some general measurable space $X$ ).

(i) There exists a measure $P_{3}$ and two measures $P_{1}^{\prime}$ and $P_{2}^{\prime}$ which are orthogonal to each other such that

$$
P_{1}=P_{3}+P_{1}^{\prime}, P_{2}=P_{3}+P_{2}^{\prime} \text {. }
$$

$$
\begin{aligned}
& P_{3}(X)=1-\sup _{A \subset X}\left|P_{1}(A)-P_{2}(A)\right| \\
& P_{1}^{\prime}(\mathrm{X})=P_{2}^{\prime}(X)=\sup _{A \subset X}\left|P_{1}(A)-P_{2}(A)\right| .
\end{aligned}
$$

These facts are verified as follows. Define the signed measure $\nu(A)$ on $\mathrm{X}$ by

$$
\nu(A)=P_{1}(A)-P_{2}(A)
$$

and let $B^{+}$and $B^{-}$be a Hahn decomposition for $\nu(A)$ so that $B^{+} \cup B^{-}$ $=X, \quad B^{+} \cap B^{-}=\varnothing, \nu\left(B^{+} \cap A\right) \geq 0, \nu\left(B^{-} \cap A\right) \leq 0$, for all $A \subset X$. Now define

(2.7.7) $\quad P_{3}(A)=\frac{1}{2}\left(P_{1}(A)+P_{2}(A)\right)-\frac{1}{2} \nu\left(B^{+} \cap A\right)+\frac{1}{2} \nu\left(B^{-} \cap A\right)$.

Since $\nu(A)=\nu\left(A \cap B^{+}\right)+\nu\left(A \cap B^{-}\right)$it follows from (2.7.7) that

$$
\begin{aligned}
P_{3}(A) & =P_{1}(A)-\nu\left(B^{\top} \cap A\right) \\
& =P_{2}(A)+\nu\left(B^{-} \cap A\right) .
\end{aligned}
$$

Define also

$$
P_{1}^{\prime}(A)=\nu\left(B^{\prime} \cap A\right), P_{2}^{\prime}(A)=-\nu\left(B^{-} \cap A\right) .
$$

From (2.7.8) and (2.7.9) it follows that (2.7.6) (i) holds and that 
$P_{1}^{\prime}$ and $P_{2}^{\prime}$ are orthogonal. To verify (ii) in (2.7.6) we note from (2. 7.8) that

$$
P_{3}(X)=1-\nu\left(B^{+}\right) .
$$

But

$$
\begin{aligned}
\sup _{A \subset X} \mid & P_{1}(A)-P_{2}(A) \mid \\
& =\sup _{A \subset X}\left|P_{1}\left(A \cap B^{+}\right)+P_{1}\left(A \cap B^{-}\right)-P_{2}\left(A \cap B^{+}\right)-P_{2}\left(A \cap B^{-}\right)\right| \\
& =\sup _{A \subset X}\left|\nu\left(B^{+} \cap A\right)+\nu\left(B^{-} \cap A\right)\right|=\nu\left(B^{+}\right) .
\end{aligned}
$$

Thus, the facts stated in (2.7.6) have been proved.

We return now to the proof of the theorem. Let $P\left(t_{0}, J_{1}, A\right)$ and $P\left(t_{0}, y_{2}^{\prime}, A\right)$ be denoted, respectively, by $P_{1}$ and $P_{2}$ on $S$ and let $P_{3}$ and $P_{1}^{\prime}, P_{2}^{\prime}$ be the other measures described in (2.7.6). From (2.7.5) and (2.7.6) it follows that

(2. 7.10$)$

$$
\begin{aligned}
P_{3}(S) & =1-\sup _{A \subset S}\left|P_{1}(A)-P_{2}(A)\right| \\
& \geq 1-\uparrow
\end{aligned}
$$

and

$$
\begin{aligned}
P_{1}^{\prime}(S) & =P_{2}^{\prime}(S)=1-P_{3}(S) \\
& =\sup _{A \subset S}\left|P_{1}(A)-P_{2}(A)\right| \leq r / 2 .
\end{aligned}
$$

Note further that

$$
\begin{aligned}
\sup _{A \subset S} \mid & P_{3}(A)-\bar{P}(A) \mid \\
& \leq \sup _{A \subset S}\left|P_{3}(A)-P_{1}(A)\right|+\sup _{A \subset S}\left|P_{1}(A)-\bar{P}(A)\right| \\
& \leq \frac{\gamma}{2}+\frac{\gamma}{4}=\frac{3 \gamma}{4} .
\end{aligned}
$$

Hence,

$$
\text { (2.7.12) } \begin{aligned}
P_{3}\{|z(y)| \geq \theta\} \\
\quad=P_{3}\{|z(y)| \geq \theta\}-\bar{P}\{|z(y)| \geq \theta\}+\bar{P}\{|z(y)| \geq \theta\} \\
\\
\geq \gamma-\frac{3 \gamma}{4}=\frac{\gamma}{4} .
\end{aligned}
$$


Now let $U(\eta, y,-1), \eta \leq 0, y \in S, A \subset S$ be the solution of (2.6.12). If $t_{0}<-\eta_{0}$ i.e., $\eta_{0}$ is sufficiently large and negative, we have (2.7.13) $\left|U\left(\eta_{0}, y_{1}, A\right)-U\left(\eta_{0}, y_{2}, A\right)\right|$

$$
\begin{gathered}
=\mid \iint U\left(\xi, y_{3} ; A\right)\left[\widetilde{P}\left(t_{0}, \eta_{0}, y_{1}, d \xi, d y_{3}\right)\right. \\
\left.-\widetilde{P}\left(t_{0}, \eta_{0}, y_{2}, d \xi, d y_{3}\right)\right] \mid \\
=\mid \int G\left(\eta_{0}, y_{1}, y_{3} ; A\right) P\left(t_{0}, y_{1}, d y_{3}\right) \\
-\int G\left(\eta_{0}, y_{3}, y_{3} ; A\right) P\left(t_{0}, y_{2}, d y_{3}\right) \mid
\end{gathered}
$$

where,

$$
\begin{gathered}
G\left(\eta_{0}, y_{1}, \jmath_{s}^{\prime} ; A\right) \\
=E\left\{U\left(H\left(t_{0}\right), y\left(t_{0}\right), A\right) \mid y\left(t_{0}\right)=y_{3}, y(0)=y_{1}^{\prime}, H(0)=\eta_{0}\right\}
\end{gathered}
$$

is a non-negative function bounded by one. Thus, using (2.7.6),

(2.7.15) $\left|U\left(\eta_{0}, y_{1}, A\right)-U\left(\eta_{0}, y_{2}, A\right)\right|$

$$
\begin{aligned}
& =\left|\int G\left(\eta_{0}, y_{1}, y_{3} ; A\right) P_{1}\left(d y_{3}\right)-\int G\left(\eta_{0}, y_{2}, y_{3} ; A\right) P_{2}\left(d y_{3}\right)\right| \\
& =\mid \int\left[G\left(\eta_{0}, y_{1}, y_{3} ; A\right)-G\left(\eta_{0}, y_{2}, y_{3} ; A\right)\right] P_{3}\left(d y_{3}\right) \\
& \quad+\int G\left(\eta_{0}, y_{1}, y_{3} ; A\right) P_{1}^{\prime}\left(d y_{3}\right) \\
& \quad-\int G\left(\eta_{0}, y_{2}, y_{3} ; A\right) P_{3}^{\prime}\left(d y_{3}\right) \mid \\
& \leq \int\left|G\left(\eta_{0}, y_{1}, y_{3} ; A\right)-G\left(\eta_{0}, y_{2}, y_{3} ; A\right)\right| P_{3}\left(d y_{3}\right) \\
& \quad+1-P_{3}(S) .
\end{aligned}
$$

We must now estimate the integrand on the right of the last inequality in $(2.7 .15)$.

From (2. 7.14) it follows that

(2. 7. 16) $\left|G\left(\eta_{0}, y_{2}, y_{3} ; A\right)-G\left(\eta_{0}, y_{2}, y_{3} ; A\right)\right|$

$$
\leq \sup _{\left|\eta_{1}-\eta_{2}\right| \leq 2 t_{0}}\left|U\left(\eta_{1}, y_{3} ; A\right)-U\left(\eta_{2}, y_{3} ; A\right)\right|
$$


where we have used the fact that $|\approx(y)| \leq 1$. Therefore it suffices to estimate $\left|U\left(\eta_{1}, y ; A\right)-U\left(\eta_{2}, y ; A\right)\right|$ and this we do directly from (2.6. 12). We have that

$$
\frac{\partial U}{\partial \eta}-\frac{q(y) U}{\approx(y)} \leq 0, \quad \eta<0
$$

or, upon integrating,

$$
U\left(\eta_{2}, y ; A\right) \leq U\left(\eta_{1}, y ; A\right) e^{q\left(\eta_{2}-\eta_{1}\right) / z(y) \mid}
$$

and hence

$$
U\left(\eta_{2}, y, A\right)-U\left(\eta_{1}, y, A\right) \leq 1-e^{-q\left|\eta_{2}-\eta_{1}\right| /|z(y)|} .
$$

Replacing $U$ by $1-U$ in this argument gives

$$
\left|U\left(\eta_{2}, y, A\right)-U\left(\eta_{1}, y, A\right)\right| \leq 1-e^{-q(y)\left|\eta_{2}-\eta_{1}\right| / z(y) \mid} .
$$

We may also replace $q(y)$ on the right hand side by $q_{u}$ from (2.5.3). We return now to (2.7.15) and use (2.7.17). This yields

(2.7.18) $\left|U\left(\eta_{0}, y_{1}, A\right)-U\left(\eta_{0}, y_{2}, A\right)\right|$

$$
\begin{aligned}
& \leq \int\left(1-e^{-2 q_{u} t_{0} /\left|z\left(y_{3}\right)\right|}\right) P\left(d y_{3}\right)+1-P_{3}(S) \\
& \leq\left(1-e^{-2 q_{u} t_{0} / \theta}\right) P_{3}\left\{\left|z\left(y_{3}\right)\right| \geq 0\right\}+P_{3}\left\{\left|z\left(y_{3}\right)\right|<0\right\}+1-P_{s}(S) \\
& \left.=1-e^{-2 q_{u} t_{0} / \theta} P_{3}\left\{\left|z\left(y_{3}\right)\right| \geq \theta\right\} \quad \text { (by }(2.7 .12)\right) \\
& \leq 1-e^{-2 q_{u} t_{0} / \theta} \frac{\gamma}{4}=\rho<1 .
\end{aligned}
$$

This completes the proof of the theorem.

We are now in a position to complete the potential theory associated with the process $(H(t), Y(t))$ on $(-\infty, 0] \times S$ and generated by $\widetilde{Q}$ of (2.6.7). We have the following result that will be used in the asymptotic analysis.

Theorem 2. Let $g(\eta, y)$ be a nonnegative function on $(-\infty, 0]$ $\times S$ such that

$$
g(\eta, y) \leq \gamma e^{i \eta}, \quad \eta \leq 0, r>0, \quad \hat{0}>0 .
$$


Let $h(y)$ be a bounded function on $S$ and consider the problem

$$
\begin{gathered}
-\left(z(y) \frac{\partial w}{\partial \eta}+Q w\right)=g, \text { on }\{\eta<0, y \in S\} \cup\left\{\eta=0, y \in S^{+}\right\}, \\
w(0, y)=h(y), y \in S^{+}
\end{gathered}
$$

(cf. (2.6.12)). If $h(y)$ is adjusted appropriately by the addition of a constant (see (2.7.41) below), then (2.7.20) has a unique bounded solution $w(\eta, y)$ that decay's exponentially fast as $\eta \rightarrow-\infty$.

Before giving the proof of this theorem we prove a lemma as follows.

Lemma. Let $g(\eta, y)$ satisfy (2.7.19). Then there exist solutions of

$$
-\left(\approx(y) \frac{\partial w}{\partial \eta}+Q w\right)=g \text {, on }\{\eta<0, y \in S\} \cup\left\{\eta=0, y \in S^{+}\right\}
$$

that are bounded for all $\eta \leq 0$.

Proof of the Lemma. We shall assume that the solution of (2.7. 21) that we seek satisfies $v(0, y)=0, y \in S^{+}$; we show that such a solution exists and is bounded.

Suppose we can find a function $u(\eta, y)$ such that

$$
0 \leq u \leq M<\infty
$$

and

$$
-\left(z(y) \frac{\partial u}{\partial \eta}+Q u\right)=h \geq g, \quad \eta \leq 0
$$

Then

$$
0 \leq w \leq u \leq M
$$

and the lemma follows. To see that (2.7.24) is true note that for any $0 \leq t<\infty$ we have 
112 Alain Bensoussan, Jacques l. Lions and George C. Papanicolaou

(2.7.25) $\quad \infty>M \geq u(\eta, y)$

$$
\begin{aligned}
& \geq E_{\eta, y}\left\{\int_{0}^{\tau \wedge t} h(H(s), Y(s)) d s\right\}+E_{\eta, y}\{u(0, Y(\tau \wedge t))\} \\
& \geq E_{\eta, y}\left\{\int_{0}^{\tau \wedge t} g(H(s), Y(s)) d s\right\}
\end{aligned}
$$

and hence (2. 7.24) also, by letting $t \uparrow \infty$. Here $\tau$ is the first time $H(t)$ reaches the origin starting from $H(0)=\eta<0$.

To construct the comparison function $u$ we proceed as follows. Let $f(\eta), \eta \leq 0$ be a smooth function to be chosen later on and define $f_{1}(\eta, v)$, $f_{2}(\eta, v)$ by

$$
\begin{aligned}
& Q f_{1}+z \frac{\partial f}{\partial \eta}=0, \\
& Q f_{2}+\approx \frac{\partial f_{1}}{\partial \eta}-\overline{\frac{\partial f_{1}}{\partial \eta}}=0 .
\end{aligned}
$$

Here and in the remainder of the proof of this lemma the overbar denotes average with respect to the invariant measure of $Q$ i.e., $\bar{P}$ of (2.5.6). Clearly $f_{1}$ and $f_{2}$ are well defined because of (2.5.9), (2.5.10) and (2.6.6). We write

$$
\begin{aligned}
& f_{1}=-Q^{-1} z \frac{\partial f}{\partial \eta} \\
& f_{2}=Q^{-1}\left(z Q^{-1} z-\overline{z Q^{-1} z}\right) \frac{\partial^{2} f}{\partial \eta^{2}} \\
& 0<\frac{1}{2} \sigma^{2}=-\overline{z Q^{-1} \approx}
\end{aligned}
$$

The positivity was assumed explicity below (2.6.6"). From these definitions it follows that

$$
\begin{aligned}
& -\left(z \frac{\partial}{\partial \eta}+Q\right)\left(f+f_{1}+f_{2}\right) \\
& =-\frac{\sigma^{2}}{2} \frac{\partial^{2} f}{\partial \eta^{2}}+z Q^{-1}\left(-z Q^{-1} z-\frac{1}{2} \sigma^{2}\right) \frac{\partial^{3} f}{\partial \eta^{3}} .
\end{aligned}
$$

Now choose $f(\eta)$ so that

$$
-\frac{\sigma^{2}}{2} \frac{\partial^{2} f}{\partial \eta^{2}}=\alpha e^{\beta \eta}, \quad \eta \leq 0,
$$


with $0<\beta<\delta$ and $0<\gamma<\alpha$ appropriately chosen. With this choice, the right hand side of (2.7.28), which is $h$ of (2.7.23), is

$$
h=\alpha\left[1-\frac{2 \beta}{\sigma^{2}} z Q^{-1}\left(-z Q^{-1} \approx-\frac{1}{2} \sigma^{2}\right)\right] e^{\beta_{\eta}} .
$$

For $\alpha$ sufficiently large and $\beta>0$ sufficiently small we achieve the inequality on the right side of (2.7.23). Integrating (2.7.29) yields

$$
f(\eta)=\frac{2}{\sigma^{2}} \frac{\alpha}{\beta^{2}}\left(A-e^{\beta \eta}\right) \geq 0, \quad \eta \leq 0
$$

where $A>0$ is sufficiently large. Moreover if $u=f+f_{1}+f_{2}$ then clearly $u \geq 0$ if $A$ is sufficiently large. This completes the proof of the lemma.

Proof of Theorem 2. Let $\left(T_{\eta} f\right)(v)$ be defined by (2.6.13). It follows from the lemma just proven that we may write the solution of (2. 7.20$)$ as

$$
\text { (2. 7. 32) } \quad r(\eta, y)=E_{\eta, y}\{h(Y(\tau))\}+E_{\eta, y}\left\{\int_{0}^{\tau} g(H(s), Y(s)) d s\right\} .
$$

Let $\tau_{1}, \tau_{2}, \cdots, \tau_{n}$ be the first times $-n+1,-n+2, \cdots, 0$ are reached starting from $\eta=-n$. Then,

$$
\begin{aligned}
\widetilde{w}(-n, y) & =E_{-n, y}\left\{\int_{0}^{\tau} g(H(s), Y(s)) d s\right\} \\
& =E_{-n, y}\left\{\int_{0}^{\tau_{1}}+\cdots+\int_{\tau_{n-1}}^{\tau_{n}} g(H(s), Y(s)) d s\right\} \\
& =E_{-n, y}\left\{\sum_{k=1}^{n} E\left\{\int_{\tau_{k-1}}^{\tau_{k}} g(H(s), Y(s)) d s \mid \mathscr{F}_{\tau_{k-1}}\right\}\right\} .
\end{aligned}
$$

Here $\mathscr{F}_{\tau_{k-1}}$ is the $\sigma$-algebra associated with the stopping time $\tau_{k-1}$. With $y \in S^{-}$define

$$
\widetilde{g}_{n}(y)=E_{-n, y}\left\{\int_{0}^{r_{1}} g(H(s), Y(s)) d s\right\}
$$

From the lemma above it follows that

$$
0 \leq \widetilde{g}_{n}(y) \leq c c^{-\delta(n-1)}
$$

for some constant $c>0$ and $\delta>0$ sufficiently small. From (2.7.33) and (2.7.34) it follows that 
(2.7.36)

$$
\widetilde{w}(-n, y)=\widetilde{g}_{n}+T_{-1} \widetilde{g}_{n-1}+T_{-1}^{2} \widetilde{g}_{n-2}+\cdots+T_{-1}^{n-1} \widetilde{g}_{1} .
$$

Let $G_{n}(y)$ be defined by

$$
G_{n}(y)=\sum_{k=1}^{n} \widetilde{g}_{k}(y) \text {. }
$$

We shall show that

$$
\lim _{n \uparrow \infty} \widetilde{w}(-n, y)=\bar{G}
$$

where

$$
\bar{G}=\int_{S^{+}} G(y) \bar{U}(d y), \quad G(y)=\lim _{n \uparrow \infty} G_{n}(y)
$$

and the limit in (2.7.38) is approached exponentially fast, uniformly in $y \in S$. Note that $G(y)$ exists since $G_{n}(y)$ is an increasing sequence and the limit $G$ is approached exponentially fast in view of (2.7.35). We write

$$
\begin{aligned}
\sum_{j=1}^{n} T_{-1}^{n-j} \widetilde{g}_{j} & =\sum_{j=1}^{k} T_{-1}^{n-j} \widetilde{g}_{j}+\sum_{j=k+1}^{n} T_{-1}^{n-j} \widetilde{g}_{j} \\
& =T_{-1}^{n-k}\left(\sum_{j=1}^{k} T_{-1}^{j-k} \widetilde{g}_{j}\right)+\sum_{j=k+1}^{n} T_{-1}^{n-j} \widetilde{g}_{j} .
\end{aligned}
$$

The second term on the right side of (2.7.40) is small uniformly in $n$ and $y$ as long as $k$ is sufficiently large. With $k$ large and fixed now we let $n \rightarrow \infty$ on the first term on the right side of (2.7.40). By the ergodicity of $T_{-1}$ it tends to

$$
\int_{S^{+}} \bar{U}(d y) \sum_{j=1}^{k}\left(T_{-1}^{k-j} \widetilde{g}_{j}\right)(y)=\int_{S^{+}} \bar{U}(d y) \sum_{j=1}^{k} \widetilde{g}_{j}(y) \sim \bar{G}
$$

and so the statement is proved.

Let $\bar{h}$ be defined by

$$
\bar{h}=\int_{S^{+}} \bar{U}(d y) h(y) .
$$

If we adjust $h(y)$ by adding a constant so that

$$
\bar{h}+\bar{G}=0 \text {, }
$$

then clearly $w(\eta, y) \rightarrow 0$ as $\eta \rightarrow-\infty$ and the approach is exponentially fast uniformly in $y \in S$. The proof of the theorem is complete. 


\section{§3. Diffusion Approximations}

\section{1. Asymptotic Expansion in an Unbounded Region and Homogenization}

We begin with the asymptotic analysis as $\varepsilon \rightarrow 0$ of the following problem (cf. Section 2.4)

$$
\begin{aligned}
& \frac{\partial u^{\varepsilon}(t, x, y)}{\partial t}=\frac{1}{\varepsilon^{2}} Q_{x,(x / \varepsilon)} u^{\varepsilon}(t, x, y) \\
& +\left(\frac{1}{\varepsilon} F\left(x, \frac{x}{\varepsilon}, y\right)+G\left(x, \frac{x}{\varepsilon}, y\right)\right) \frac{\partial u^{\varepsilon}(t, x, y)}{\partial x} \\
& +a\left(x, \frac{x}{\varepsilon}, y\right) u^{\varepsilon}(t, x, y), \\
& \quad t>0, x \in \mathbb{R}^{n}, y \in S \\
& u^{\varepsilon}(0, x, y)=f\left(x, \frac{x}{\varepsilon}, y\right), \quad x \in \mathbb{R}^{n}, y \in S .
\end{aligned}
$$

Here $F(x, \zeta, y)$ and $G(x, \zeta, y)$ are smooth bounded functions on $\mathbb{R}^{n} \times T^{n}$ $\times S \rightarrow \boldsymbol{R}^{n}$, where $T^{n}$ is the unit $n$-dimensional torus and $S$ is any compact metric space (such as the unit sphere in $\mathbb{R}^{n}$ for example); $a(x, \zeta, y)$ is a smooth bounded function on $\mathbb{R}^{n} \times T^{n} \times S \rightarrow \mathbb{R}$. The operator $Q_{x, \zeta}$, with parameters $x \in \mathbb{R}^{n}$ and $\zeta_{s} \in T^{n}$, is given by

$$
Q_{x, \zeta} f(y)=q(x, \zeta, y) \int_{s} \pi(x, \zeta, y, d z) f(z)-q(x, \zeta, y) f(y),
$$

where $q$ is a continuous function on $\mathbb{R}^{n} \times T^{n} \times S$ such that

$$
0<q_{l} \leq q(x, \zeta, y) \leq q_{u}<\infty,
$$

and $\pi(x, \zeta, y, A), A$ a Borel subset of $S$, is a probability measure for each $(x, \zeta, y)$ on $\mathbb{R}^{n} \times T^{n} \times S$ and is continuous in $(x, \zeta, y)$ for each Borel set $A$. Under these conditions (3.1.1) has the probabilistically natural (generalized) solution

$$
\begin{aligned}
u^{\varepsilon}(t, x, y)= & E_{x, y}\left\{\exp \left(\int_{0}^{t} a\left(X^{\varepsilon}(s), \frac{X^{\varepsilon}(s)}{\varepsilon}, Y^{\varepsilon}(s)\right) d s\right)\right. \\
& \left.\times f\left(X^{\varepsilon}(t), \frac{X^{\varepsilon}(t)}{\varepsilon}, Y^{\varepsilon}(t)\right)\right\}
\end{aligned}
$$


for bounded and continuous $f(x, \zeta, y)$. Here $\left(X^{\varepsilon}(t), Y^{\varepsilon}(t)\right), \varepsilon>0$ fixed, is the corresponding process on $\mathbb{R}^{n} \times S$. If the $x$ and $\zeta\left(=\frac{x}{\varepsilon}\right)$ dependence of coefficients and data is smooth then $\iota^{\varepsilon}(t, x, y)$ is smooth also and satisfies (3.1.1) in the usual way. We shall assume that this is the case in what follows.

We wish to describe the asymptotic behavior of $\iota^{\varepsilon}(t, x, y)$ as $\varepsilon \rightarrow 0$, $0 \leq t \leq T<\infty$ and to analyze the corresponding process $\left(X^{\varepsilon}(t), Y^{\varepsilon}(t)\right)$. To this end, and under liberal smoothness assumptions, we first construct an asymptotic expansion for $u^{\varepsilon}(t, x, y)$. In Section 3.2 we prove that this is a valid asymptotic expansion and in Section 3.3 we show that $X^{c}(t), 0 \leq t \leq T$, converges weakly as a process on $C\left([0, T] ; \mathbb{R}^{n}\right)$ to a diffusion Markov process.

Let us introduce explicitly the variables

$$
\zeta=\frac{x}{\varepsilon}, \tau=\frac{t}{\varepsilon^{2}}
$$

and let us seek an expansion of the form

$$
\begin{aligned}
u^{\varepsilon}(t, x, y)= & \left.\sum_{k=0}^{\infty} \varepsilon^{k} u_{k}(t, x, \zeta, y)\right|_{\xi=x / \varepsilon} \\
& +\left.\sum_{k=0}^{\infty} \varepsilon^{k} u_{k}^{I L}(\tau, x, \zeta, y)\right|_{\substack{\tau=t / \varepsilon^{2} \\
\zeta=x / \varepsilon}}
\end{aligned}
$$

Here the superscript IL stands for "initial layer" and reflects the nature of such terms on the right side of (3.1.6) as we see below. We require that both sums on the right side of (3.1.6) satisfy (3.1.1) and that their sum satisfies $\left(3.1 .1^{\prime}\right)$. Thus, we have at first the following sequence of problems ${ }^{\dagger}$ for the interior expansion ( $u_{k}$ without superscripts).

$$
\begin{gathered}
\mathcal{L}_{1} u_{0}=0 \\
\mathcal{L}_{1} u_{1}+\mathcal{L}_{2} u_{0}=0 \\
\mathcal{L}_{1} u_{N}+\mathcal{L}_{2} u_{N-1}+\mathcal{L}_{3} u_{N-2}-\frac{\partial u_{N-2}}{\partial t}=0, \quad N=2,3,4, \cdots .
\end{gathered}
$$

Here $\mathcal{L}_{1}, \mathcal{L}_{2}$ and $\mathcal{L}_{3}$ are defined by

${ }^{\dagger}$ In inserting (3.1.6) into (3.1.1) and equating coefficients of equal powers of $\varepsilon$, we treat $x, \zeta$ and $t, \tau$ as independent variables. Thus, $(\partial / \partial x) \rightarrow(\partial / \partial x)+(1 / \varepsilon)(\partial / \partial \zeta)$. 


$$
\begin{aligned}
& \mathcal{L}_{1}=Q_{x, \zeta}+F(x, \zeta, y) \cdot \frac{\partial}{\partial \zeta} \\
& \mathcal{L}_{2}=F(x, \zeta, y) \cdot \frac{\partial}{\partial x}+G(x, \zeta, y) \cdot \frac{\partial}{\partial \zeta} \\
& \mathcal{L}_{3}=G(x, \zeta, y) \cdot \frac{\partial}{\partial x}+a(x, \zeta, y) .
\end{aligned}
$$

We take $u_{0}$ independent of $y$ and $\zeta$ so that $u_{0}=u_{0}(t, x)$ satisfies (3.1.7). We will find the determining equation for $\iota_{0}$ later. We consider next equation (3.1.8). The operator $\mathcal{L}_{1}$ is an operator on functions $T^{n} \times S$ with $x$ a fixed parameter. It is precisely of the form (2.5.2) for which we have studied the validity of the Fredholm alternative in Section 2.5. The theorem at the end of Section 2.5 deals with the case $S=$ unit sphere in $\mathbb{R}^{n}$ and $F=y$; this is the case of interest in transport theory. We shall assume that the Fredholm alternative, as stated in that theorem, is valid for $\mathcal{L}_{1}$ of (3.1.10) in order to get a better idea of the structure of the expansion. The remark at the end of Section 2.5 clarifies the smooth dependence of quantities on the parameter $x$.

Let $\tilde{\bar{P}}(d y d \zeta ; x)$ be the invariant measure ${ }^{\dagger}$ of $\mathcal{L}_{1}$. We shall assume that

$$
\int_{T^{n} \times S} F(x, \zeta, y) \tilde{\bar{P}}(d y d \zeta ; x) \equiv 0
$$

i.e., that the singular velocity term in (3.1.1) averages to zero relative to the invariant measure of the cell-collision operator $\mathcal{L}_{1}$. Condition (3.1.13) and the Fredholm alternative for $\mathcal{L}_{1}$ yield the solution $u_{1}$ of (3.1.8) in the form

$$
u_{1}=u_{10}-\mathcal{L}_{1}^{-1} \mathcal{L}_{2} u_{0}
$$

or more explicitly

$$
\begin{aligned}
& u_{1}(t, x, \zeta, y)=u_{10}(t, x) \\
& \quad+\int_{T^{n} \times S} \widetilde{\psi}\left(y, \zeta, d y^{\prime}, d \zeta^{\prime} ; x\right) F\left(x, \zeta^{\prime}, y^{\prime}\right) \cdot \frac{\partial u_{0}(t, x)}{\partial x} .
\end{aligned}
$$

\footnotetext{
† We use $\sim$ to distinguish objects on $T^{n} \times S$ from the corresponding ones on $S$ alone (i.e., without fast periodic structure).
} 
Here $\widetilde{\psi}$ is the recurrent potential kernel of $\mathcal{L}_{1}$ that is, if $\widetilde{P}(t, y, \zeta, \widetilde{A} ; x)$, $\widetilde{A} \subset T^{n} \times S$ is the transition function corresponding to $\mathcal{L}_{1}$ (the kernel of $\left.\exp \left(t \mathcal{L}_{1}\right)\right)$ then,

$$
\widetilde{\psi}(y, \zeta, \tilde{A} ; x)=\int_{0}^{\infty}[\widetilde{P}(t, y, \zeta, \tilde{A} ; x)-\tilde{\bar{P}}(\tilde{A})] d t
$$

The function $u_{10}(t, x)$, like $u_{0}(t, x)$ is determined later.

We use next (3.1.14) in (3.1.9), with $N=2$, and rewite it in the form

$$
\mathcal{L}_{1} u_{2}=-\mathcal{L}_{2}\left(u_{10}-\mathcal{L}_{1}^{-1} \mathcal{L}_{2} u_{0}\right)-\mathcal{L}_{3} u_{0}+\frac{\partial u_{0}}{\partial t}
$$

Let us denote by overbar integration of a quantity with respect to $\tilde{\bar{P}}$. Then the solvability condition for $u_{2}$ is clearly

$$
\frac{\partial u_{0}}{\partial t}=\left(\overline{-\mathcal{L}_{2} \mathcal{L}_{1}^{-1} \mathcal{L}_{2}+\mathcal{L}_{3}}\right) u_{0} \equiv \overline{\mathcal{L}} u_{0}
$$

Note that the term $-\mathcal{L}_{2} l_{10}$ drops out upon averaging in view of (3.1.13). Explicitly the operator $\overline{\mathcal{L}}$ on the right side of (3.1.17) has the form (3. 1. 18) $\overline{\mathcal{L}} g(x)$

$$
\begin{aligned}
= & \int_{T^{n} \times S} \tilde{\widetilde{P}}(d \zeta d y ; x)\left[F(x, \zeta, y) \cdot \frac{\partial}{\partial x}+G(x, \zeta, y) \cdot \frac{\partial}{\partial \zeta}\right] \\
& \times \int_{T^{n} \times S} \widetilde{\psi}\left(y, \zeta, d y^{\prime}, d \zeta^{\prime} ; x\right) F\left(x, \zeta^{\prime}, y^{\prime}\right) \cdot \frac{\partial g(x)}{\partial x} \\
& +\int_{T^{n} \times S} \widetilde{\bar{P}}(d \zeta d y ; x)\left[G(x, \zeta, y) \cdot \frac{\partial g(x)}{\partial x}+a(x, \zeta, y) g(x)\right] .
\end{aligned}
$$

This is an elliptic second order differential operator and so (3.1.17) is a parabolic equation for $u_{0}(t, x)$. Initial conditions for $u_{0}$ will be obtained from the initial layer analysis later.

The solution $u_{2}$ of (3.1.9) (with $N=2$ ) takes the form

$$
u_{2}=u_{20}-\mathcal{L}_{1}^{-1} \mathcal{L}_{2} u_{10}-\mathcal{L}_{1}^{-1}\left(-\mathcal{L}_{2} \mathcal{L}_{1}^{-1} \mathcal{L}_{2}+\mathcal{L}_{3}-\overline{\mathcal{L}}\right) u_{0},
$$

where $u_{20}-u_{20}(t, x)$ is determined by the solvability condition for (3. 1. 9) with $N=4$. The solvability condition for (3.1.9) with $N=3$ determines (up to initial data) the function $\iota_{10}(t, x)$ as we now show. We have 


$$
\begin{aligned}
\mathcal{L}_{1} u_{3}= & -\mathcal{L}_{2} u_{2}-\mathcal{L}_{3} u_{1}+\frac{\partial u_{1}}{\partial t} \\
= & \mathcal{L}_{2}\left[\mathcal{L}_{1}^{-1} \mathcal{L}_{2} u_{10}+\mathcal{L}_{1}^{-1}\left(-\mathcal{L}_{2} \mathcal{L}_{1}^{-1} \mathcal{L}_{2}+\mathcal{L}_{3}-\overline{\mathcal{L}}\right) u_{0}-u_{20}\right] \\
& +\mathcal{L}_{3} \mathcal{L}_{1}^{-1} \mathcal{L}_{2} u_{0}-\mathcal{L}_{3} u_{10}-\mathcal{L}_{1}^{-1} \mathcal{L}_{2} \overline{\mathcal{L}} u_{0}+\frac{\partial u_{10}}{\partial t} \\
= & \left(\mathcal{L}_{2} \mathcal{L}_{1}^{-1} \mathcal{L}_{2}-\mathcal{L}_{3}\right) u_{10}+\frac{\partial u_{10}}{\partial t} \\
& +\mathcal{L}_{2} \mathcal{L}_{1}^{-1}\left(-\mathcal{L}_{2} \mathcal{L}_{1}^{-1} \mathcal{L}_{2}+\mathcal{L}_{3}-\overline{\mathcal{L}}\right) u_{0}+\mathcal{L}_{3} \mathcal{L}_{1}^{-1} \mathcal{L}_{2} u_{0}-\mathcal{L}_{2} u_{20},
\end{aligned}
$$

from which we obtain the following equation for $u_{10}(t, x)$

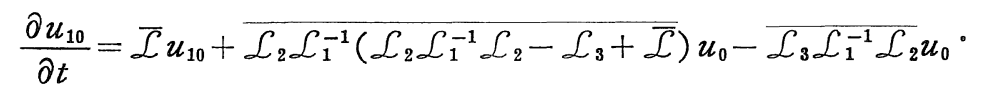

This is an inhomogeneous diffusion equation with inhomogeneous term depending on $u_{0}$. Initial data are determined below from the initial layer expansion.

The higher order terms in the interior expansion in (3.1.6) (not the initial layer terms) are obtained in the same fashion up to initial conditions. Each time $u_{N}$ is solved from (3.1.9) an unknown function $u_{N 0}(t, x)$ is introduced and this is in turn determined (up to initial values) by the solvability condition for $u_{N+2}$.

Now we consider the initial layer expansion. Inserting $\sum_{k_{k=0}^{\infty}}^{\infty} \varepsilon^{k} u_{k}^{I L}$ into (3.1.1), treating $\zeta$ and $\tau$ as independent variables, and equating coefficients of equal powers of $\varepsilon$ leads to the following problems.

$$
\begin{gathered}
\frac{\partial u_{0}^{I L}}{\partial \tau}=\mathcal{L}_{1} u_{0}^{I L} \\
\frac{\partial u_{1}^{I L}}{\partial \tau}=\mathcal{L}_{1} u_{1}^{I L}+\mathcal{L}_{2} u_{0}^{I L} \\
\frac{\partial u_{N}^{I L}}{\partial \tau}=\mathcal{L}_{1} u_{N}^{I L}+\mathcal{L}_{2} u_{N-1}^{I L}+\mathcal{L}_{3} u_{N-2}^{I L}, \quad N=2,3,4, \cdots .
\end{gathered}
$$

We require also that

$$
u_{N}^{I L}(\tau, x, \zeta, y) \rightarrow 0 \text { as } \tau \rightarrow \infty, \quad N=0,1,2, \cdots
$$

and, from (3.1. $\left.1^{\prime}\right)$, that

$$
u_{0}(0, x, \zeta, y)+u_{0}^{I L}(0, x, \zeta, y)=f(x, \zeta, y),
$$




$$
u_{N}(0, x, \zeta, y)+u_{N}^{I L}(0, x, \zeta, y)=0, \quad N=1,2, \cdots .
$$

Clearly (3.1.24) is the condition that we have an initial layer behavior and (3.1.25), (3.1.26) are the initial conditions from which we will determine initial values for $u_{0}, u_{10}, u_{20}, \cdots$.

From (3.1.21), (3.1.25) and the ergodic properties of $\mathcal{L}_{1}$ it is clear that $u_{0}^{I L}$ will go to zero* as $\multimap \rightarrow \infty$ provided we have

$$
u_{0}^{I L}(0, x, \zeta, y)=f(x, \zeta, y)-\bar{f}(x)
$$

where,

$$
\bar{f}(x)=\int_{T^{n} \times S} \tilde{\bar{P}}(d \zeta d y ; x) f(x, \zeta, y)
$$

and hence

$$
u_{0}(0, x)=\bar{f}(x)
$$

Thus, (3.1.17) and (3.1.29) determine $u_{0}(t, x)$ completely and similarly (3.1.21) and (3.1.27) determine $u_{0}^{I L}=u_{0}^{I L}(\tau, x . \zeta, y)$ completely (with $x$ being a parameter in the $u_{0}^{I L}$ problem).

To determine $u_{1}$ (i.e., initial data for $u_{10}$ ) and $u_{1}^{I L}$ we note that from (3.1.22) and (3.1.26) (in operator notation)

$$
\begin{aligned}
u_{1}^{I L}(\tau)= & -e^{\mathcal{L}_{1} \tau} u_{1}(0)+\int_{0}^{\tau} e^{\mathcal{L}_{1}(\tau-s)} \mathcal{L}_{2} u_{0}^{I L}(s) d s \\
= & -e^{\mathcal{L}_{1} \tau}\left(u_{10}(0)-\mathcal{L}_{1}^{-1} \mathcal{L}_{2} u_{0}(0)\right)+\int_{0}^{\tau} \overline{\mathcal{L}_{2} u_{0}^{I L}(s)} d s \\
& +\int_{0}^{\tau} e^{\mathcal{L}_{1}(\tau-s)}\left(\mathcal{L}_{2} u_{0}^{I L}(s)-\overline{\mathcal{L}_{2} u_{0}^{I L}(s)}\right) d s .
\end{aligned}
$$

The last term on the right in (3.1.30) goes to zero as $\tau \uparrow \infty$ hence, in order that $u_{1}^{I L}(\tau) \rightarrow 0$ as $\tau \rightarrow \infty$ we must have

$$
u_{10}(0)=\overline{\mathcal{L}_{1}^{-1} \mathcal{L}_{2} u_{0}(0)}+\int_{0}^{\infty} \overline{\mathcal{L}_{2} u_{0}^{I L}(s)} d s .
$$

The initial condition (3.1.31) for $u_{10}(t, x)$ and (3.1.20) determine it completely. Similarly, (3.1.22) and (3.1.26) $(N=1)$ determine $u_{1}^{I L}$ and it satisfies (3.1.24) (exponentially fast). Thus the second terms in the expansion have been determined. The rest of the terms are calcu-

$* u_{0}^{I L}$ actually goes to zero exponentially fast as $\tau \rightarrow \infty$. 
lated by the same general process.

When (3.1.1) has no rapidly varying periodic terms, i.e., $x / \varepsilon$ does not appear in $F, G, a, Q$, and $f$, then the above expansion yields the standard diffusion approximation of transport theory [ 1 and the references therein]. On the right side of (3.1.1) we do not have terms with $y$ derivatives (force terms, whenever this makes sense) but the formalism extends obviously to this case.

\subsection{Validity of the Expansion}

The validity of the expansion (3.1.6) rests upon the following three general ingredients which have been mentioned already.

(i) Smoothness

(ii) The Fredholm alternative for $L_{1}$ of (3.1.10)

(iii) The centering condition (3.1.13).

Smoothness is inevitable if we want expansions and error estimates. The Fredholm alternative for $\mathcal{L}_{1}$ must also hold one way or another; it is the essence of the expansion procedure. The centering condition (3.1.13) can be removed but then the expansion (3.1.6) changes form and in particular $u_{0}$ depends on $\varepsilon$ in a nontrivial way.

The result we state below (and results in other sections to follow) follows easily from hypotheses of the form (i), (ii), and (iii) above. The results ${ }^{\dagger}$ of Section 2.5 on the Fredholm alternative are not intended for literal use but to show that (ii) is nonvacuous in the present context. We state the results in some generality assuming that (i), (ii), and (iii) do hold.

Let us return to (3.1.1) and assume that coefficients and data depend smoothly on $x$ and $\zeta$. We also assume that

(3.2.1) The Fredholm alternative as in the theoren at the end of Section 2.5 is valid for $\mathcal{L}_{1}$ of $(3.1 .10)$.

The coefficients of the diffusion equation (3.1.17) being smooth by hypotheses and $\bar{f}(x)$ being smooth imply that the solution $u_{0}(t, x)$ is also smooth. Similarly, $u_{0}^{I L}$ is smooth and so on.

\footnotetext{
T The theorem at the end of Section 2. 5.
} 
Theorem. Under the above hypotheses, the construction of the expansion (3.1.6) gives an asymptotic expansion of $u^{\varepsilon}(t, x, y)$ as $\varepsilon \rightarrow$ $0,0 \leq t \leq T<\infty, x \in \boldsymbol{R}^{n}, y \in S$. Specifically, we have that uniformly in $x \in \boldsymbol{R}^{n}, y \in S, N=0,1,2, \cdots$

$$
\begin{aligned}
& \left|u^{\delta}(t, x, y)-\sum_{k=0}^{N} \varepsilon^{k} u_{k}\left(t, x, \frac{x}{\varepsilon}, y\right)-\sum_{k=0}^{N} \varepsilon^{k} u_{k}^{I L}\left(\frac{t}{\varepsilon^{2}}, x, \frac{x}{\varepsilon}, y\right)\right| \\
& =O\left(\varepsilon^{N+1}\right) .
\end{aligned}
$$

Proof. The construction of (3.1.6) makes sense since all is smooth. By construction we have that

$$
\begin{aligned}
{\left[\frac{\partial}{\partial t}-\frac{1}{\varepsilon^{2}} Q_{x,(x / \varepsilon)}\right.} & \left.-\left(\frac{1}{\varepsilon} F+G\right) \cdot \frac{\partial}{\partial x}-a\right]\left(u^{\varepsilon}-\sum_{k=0}^{N} \varepsilon^{k}\left(u_{k}+u_{k}^{I L}\right)\right) \\
= & O\left(\varepsilon^{N-1}\right)
\end{aligned}
$$

and

$$
\left.\left(u^{\varepsilon}-\sum_{k=0}^{N} \varepsilon^{k}\left(u_{k}+u_{k}^{I L}\right)\right)\right|_{t=0}=0 \text { 。 }
$$

By the maximum principle (recall $a$ is bounded and $0 \leq t \leq T<\infty$ ) it follows that

$$
\left|u^{\varepsilon}-\sum_{k=0}^{N} \varepsilon^{k}\left(u_{k}+u_{k}^{I L}\right)\right|=O\left(\varepsilon^{N-1}\right), \quad N=0,1,2, \cdots .
$$

But,

$$
\begin{aligned}
& \left|u^{\varepsilon}-\sum_{k=0}^{N} \varepsilon^{k}\left(u_{k}+u_{k}^{I L}\right)\right| \\
& \quad \leq\left|u^{\varepsilon}-\sum_{k=0}^{N+2} \varepsilon^{k}\left(u_{k}+u_{k}^{I L}\right)\right|+\varepsilon^{N+1}\left|u_{N+1}+\varepsilon u_{N+2}+u_{N+1}^{I L}+\varepsilon u_{N+2}^{I L}\right| \\
& \quad=O\left(\varepsilon^{N+1}\right) .
\end{aligned}
$$

This completes the proof of the theorem.

\section{3. Weak Convergence of the Process}

Let $\mathcal{L}^{\varepsilon}$ denote the infinitesimal generator of the process $\left(X^{\varepsilon}(t)\right.$, $\left.Y^{\varepsilon}(t)\right)$, the one associated with (3.1.1): 


$$
\mathcal{L}^{\varepsilon}=\left[\frac{1}{\varepsilon} F\left(x, \frac{x}{\varepsilon}, y\right)+G\left(x, \frac{x}{\varepsilon}, y\right)\right] \cdot \frac{\partial}{\partial x}+\frac{1}{\varepsilon^{2}} Q_{x,(x / \varepsilon)} .
$$

The state space of the process is $\boldsymbol{R}^{n} \times S$, where $S$ is a compact separable metric space and $Q_{x, 5}, \zeta=\frac{x}{\varepsilon}$, is defined by (3.1.2). The purpose of this section is to show that $X^{\varepsilon}(t), 0 \leq t \leq T<\infty$, converges weakly as a measure on $C\left([0, T] ; \boldsymbol{R}^{n}\right)$ as $\varepsilon \rightarrow 0$, to the diffusion Markov process $X(t)$ whose generator is $\mathcal{L}$, given by $(3.1 .18)$ with $a \equiv 0$. The smoothness requirements of Section 3.1 and 3.2 will be weakened considerably here. However, we only show weak convergence.

The hypotheses for this section are as follows. $Q_{x, \zeta}$ is defined by (3. 1. 2) and $q(x, \zeta, y), \pi(x, \zeta, y, A)$ are continuous in $(x, \zeta, y) \in \mathbb{R}^{n} \times T^{n}$ $\times S$, have bounded $x$ and $\zeta$ derivatives and the Fredholm alternative ${ }^{\dagger}$ holds, i.e., $\widetilde{\psi}$ of (3.1.16) is well defined, it is differentiable in $x$ and takes bounded continuous functions of $\zeta, y$ into bounded continuous functions of $\zeta, y$. In addition, it maps differentiable functions of $\zeta$ to differentiable functions of $\zeta$.

The vector functions $F(x, \zeta, y)$ and $G(x, \zeta, y)$ are assumed continuous and bounded on $\boldsymbol{R}^{n} \times T^{n} \times S \rightarrow \boldsymbol{R}^{n}$ and with bounded $x$ and $\zeta$ derivatives. We assume (3.1.13) holds.

With these hypotheses, for $\varepsilon>0 \mathcal{L}^{\varepsilon}$ of (3.3.1) is indeed the generator of a Markov process as described in Section 2.1.

Let $f(x)$ be a smooth bounded function and let $f_{1}(x, \zeta, y)$ be a bounded solution of

$$
\begin{aligned}
Q_{x, \zeta} f_{1}(x, \zeta, y) & +F(x, \zeta, y) \cdot \frac{\partial f_{1}(x, \zeta, y)}{\partial \zeta} \\
& +F(x, \zeta, y) \cdot \frac{\partial f(x)}{\partial x}=0
\end{aligned}
$$

so that*

$$
f_{1}(x, \zeta, y)=\int_{T^{n} \times S} \widetilde{\psi}\left(y, \zeta, d y^{\prime}, d \zeta^{\prime} ; x\right) F\left(x, \zeta^{\prime}, y^{\prime}\right) \cdot \frac{\partial f(x)}{\partial x} .
$$

Define $\overline{\mathcal{L}}$ on smooth functions by $(3.1 .18$ ) (with $a \equiv 0$ ) or again

${ }^{f}$ As in the theorem all the end of Section 2.5 with $y$ replaced by $F(x, \zeta, y)$ ( $x$ a parameter) i.e., (3.2.1).

* $\widetilde{\psi}$ is defined by (3.1.16). 


$$
\begin{aligned}
\overline{\mathcal{L}} f(x)= & \int_{T^{n} \times S} \tilde{\bar{P}}(d \zeta d y ; x)\left[F(x, \zeta, y) \cdot \frac{\partial f_{1}(x, \zeta, y)}{\partial x}\right. \\
& \left.+G(x, \zeta, y) \cdot \frac{\partial f_{1}(x, \zeta, y)}{\partial \zeta}+G(x, \zeta, y) \cdot \frac{\partial f(x)}{\partial x}\right]
\end{aligned}
$$

We assume that $\bar{L}$ has continuous and bounded coefficients and that it is uniformly elliptic.

Theorem. Under the above hypotheses $X^{c}(t), 0 \leq t \leq T<\infty$, converges rueakly as $\varepsilon \rightarrow 0$ to the diffusion Markov process generated by $\overline{\mathcal{L}}$.

Proof. First we note [18] that $\overline{\mathcal{L}}$ indeed generates uniquely a diffusion Markov process on $\boldsymbol{R}^{n}$. In addition $X^{\varepsilon}(t), \varepsilon>0$, is continuous with probability one. The proof is in two steps. First we show compactness of $X^{\varepsilon}$ in $D\left([0, T] ; \boldsymbol{R}^{n}\right)$ and this suffices since $X^{\varepsilon}$ and the limit $X$ are in $C[19$ p. 150]. Second we identify the limit processes by showing that they solve the martingale problem associated with $\bar{L}$ [18]. Since the latter has a unique solution under our hypotheses the proof is complete.

Let $f(x)$ be a smooth function and define $f_{1}$ by (3.3.3). Put

$$
f^{\varepsilon}(x, \check{s}, y)=f(x)+\varepsilon f_{1}(x, \zeta, y) \text {. }
$$

It follows that

$$
\begin{aligned}
& \mathcal{L}^{\varepsilon} f^{\varepsilon}\left(x, \frac{x}{\varepsilon}, y\right)=\overline{\mathcal{L}} f(x) \\
& \quad+\left[A_{f}(x, \zeta, y)+\varepsilon G(x, \zeta, y) \cdot \frac{\partial f_{1}(x, \zeta, y)}{\partial x}\right]_{\zeta=x / \varepsilon}
\end{aligned}
$$

where

$$
\begin{aligned}
& A_{f}(x, \zeta, y)=F(x, \zeta, y) \cdot \frac{\partial f_{1}(x, \zeta, y)}{\partial x} \\
& \quad+G(x, \zeta, y) \cdot \frac{\partial f_{1}(x, \zeta, y)}{\partial \zeta}+G(x, \zeta, y) \cdot \frac{\partial f(x)}{\partial x}-\overline{\mathcal{L}} f(x) .
\end{aligned}
$$

Thus, $A_{f}\left(x, \zeta, y^{\prime}\right)$ is a bounded continuous function on $\boldsymbol{R}^{n} \times T^{n} \times S$ and 


$$
\int_{T^{n} \times S} \tilde{\bar{P}}(d \zeta, d y ; x) A_{f}(x, \zeta, y)=0
$$

Define $M_{f}^{e}$ by

$$
\begin{aligned}
M_{f}^{\varepsilon}(t)= & f^{\varepsilon}\left(X^{\varepsilon}(t), \frac{X^{\varepsilon}(t)}{\varepsilon}, Y^{\varepsilon}(t)\right)-f^{\varepsilon}\left(x, \frac{x}{\varepsilon}, y\right) \\
& -\int_{0}^{t} \mathcal{L}^{\varepsilon} f^{\varepsilon}\left(X^{\varepsilon}(s), \frac{X^{\varepsilon}(s)}{\varepsilon}, Y^{\varepsilon}(s)\right) d s .
\end{aligned}
$$

From the analysis of Section 2.1 it follows that $M_{f}^{\varepsilon}(t)$ is a bounded right-continuous, zero mean martingale relative to $\mathscr{F}_{t}$, the $\sigma$-algebra associated with the process $\left(X^{s}(\cdot), Y^{s}(\cdot)\right)$ up to time $t$. The increasing process $^{\dagger}\left\langle M_{f}^{\varepsilon}(t)\right\rangle$ associated with this martingale is given by

$$
\begin{aligned}
\left\langle M_{f}^{\varepsilon}(t)\right\rangle & =\int_{0}^{t}\left[\mathcal{L}^{\varepsilon}\left(f^{\varepsilon}\right)^{2}-2 f^{\varepsilon} \mathcal{L}^{\varepsilon} f^{\varepsilon}\right]\left(X^{\varepsilon}(s), \frac{X^{\varepsilon}(s)}{\varepsilon}, Y^{\varepsilon}(s)\right) d s \\
& =\int_{0}^{t}\left[Q f_{1}^{2}-2 f_{1} Q f_{1}\right]\left(X^{\varepsilon}(s), \frac{X^{\varepsilon}(s)}{\varepsilon}, Y^{\varepsilon}(s)\right) d s \\
& \equiv \int_{0}^{t} H_{f}\left(X^{\varepsilon}(s), \frac{X^{\varepsilon}(s)}{\varepsilon}, Y^{\varepsilon}(s)\right) d s,
\end{aligned}
$$

where we have omitted subscripts on $Q$ for simplicity. The second equality here follows from the fact that the derivative operators in $\mathcal{L}^{s}$ are of first order and cancell out. Also, the factor $\varepsilon^{-2}$ in front of $Q$ cancells with the $\varepsilon$ factors in $f^{\varepsilon}=f+\varepsilon f_{1}$ since $Q f=0 \quad(f$ is independent of $y$ and $\zeta)$.

The increasing process $\left\langle M_{f}^{\varepsilon}(t)\right\rangle$ is defined by the condition that $\left(M_{f}(t)\right)^{2}-\left\langle M_{f}^{\varepsilon}(t)\right\rangle$ be a martingale also. It can be verified by direct computation that the expression (3.3.10) satisfies this definition. We also have that

$$
\begin{gathered}
E\left\{\left(M_{f}^{\varepsilon}(t)-M_{f}^{\varepsilon}(s)\right)^{2} \mid \mathscr{F}_{s}\right\}=E\left\{\left\langle M_{f}^{\varepsilon}(t)\right\rangle-\left\langle M_{f}^{\varepsilon}(s)\right\rangle \mid \mathscr{F}_{s}\right\} \\
0 \leq s \leq t<\infty
\end{gathered}
$$

Let us rewrite (3.3.9) using (3.3.5) and (3.3.6). We obtain (3. 3. 12) $f\left(X^{\varepsilon}(t)\right)-f(x)-\int_{0}^{t} \overline{\mathcal{L}} f\left(Y^{\varepsilon}(s)\right) d s$

\footnotetext{
${ }^{\dagger}$ For simplicity we write $\left\langle M_{f}^{\varepsilon}\right\rangle$ instead of $\left\langle M_{f}^{\varepsilon}, M_{f}^{\varepsilon}\right\rangle$.
} 


$$
\begin{aligned}
=M_{f}^{\varepsilon}(t) & +\varepsilon\left[f_{1}\left(x, \frac{x}{\varepsilon}, y\right)-f_{1}\left(X^{\varepsilon}(t), \frac{X^{\varepsilon}(t)}{\varepsilon}, Y^{\varepsilon}(t)\right)\right] \\
& +\int_{0}^{t} A_{f}\left(X^{\varepsilon}(s), \frac{X^{\varepsilon}(s)}{\varepsilon}, Y^{\varepsilon}(s)\right) d s \\
& +\varepsilon \int_{0}^{t} G \cdot \frac{\partial f_{1}}{\partial x}\left(X^{\varepsilon}(s), \frac{X^{\varepsilon}(s)}{\varepsilon}, Y^{\varepsilon}(s)\right) d s .
\end{aligned}
$$

Let us also give an explicit expression for $H_{f}$ in (3.3.10). It is easily seen that, in fact,

$$
\begin{aligned}
& H_{f}(x, \zeta, y)=q(x, \zeta, y) \int_{S} \pi\left(x, \zeta, y, d y^{\prime}\right) \\
& \times\left[\int_{T^{n} \times S}\left[\widetilde{\psi}\left(\zeta, y^{\prime}, d \zeta^{\prime} d y^{\prime \prime} ; x\right)-\widetilde{\psi}\left(\zeta, y, d \zeta^{\prime} d y^{\prime \prime} ; x\right)\right]\right. \\
& \left.\quad \cdot F\left(x, \zeta^{\prime}, y^{\prime \prime}\right) \cdot \frac{\partial f(x)}{\partial x}\right]^{2},
\end{aligned}
$$

and this is a bounded and continuous function of its arguments.

Now we go to step one of the proof: weak compactness of $X^{\varepsilon}$ in $D$. We choose $f(x)$ to be $x_{i}, i=1,2, \cdots, n$, the coordinate functions successively. These functions are unbounded but $\int_{1}, \mathcal{L}^{\varepsilon} f^{\varepsilon}, A_{f}$ and $H_{f}$ are bounded. Let

$$
b_{i}(x)=\overline{\mathcal{L}} x_{i}, \quad i=1,2, \cdots, n,
$$

so that the vector function $b(x)$ is the drift of the diffusion operator $\overline{\mathcal{L}}$. From (3.3.12) we obtain

$$
\begin{aligned}
X_{i}^{\varepsilon}(t)=x_{i} & +\int_{0}^{t} b_{i}\left(X^{\varepsilon}(s)\right) d s-M_{i}^{\varepsilon}(t) \\
& +\int_{0}^{t} A_{i}\left(X^{\varepsilon}(s), \frac{X^{\varepsilon}(s)}{\varepsilon}, I^{\varepsilon}(s)\right) d s+O(\varepsilon)
\end{aligned}
$$

where $M_{i}^{\varepsilon}(t)$ and $A_{i}(x, \zeta, y)$ stand for $M_{f}^{\varepsilon}$ and $A_{f}$ with $f(x)=x_{i}$. Define:

$$
\begin{aligned}
\widetilde{X}_{i}^{\varepsilon}(t)=x_{i} & +\int_{0}^{t} b_{i}\left(Y^{\varepsilon}(s)\right) d s+M_{i}^{\varepsilon}(t) \\
& +\int_{0}^{t} A_{i}\left(X^{\varepsilon}(s), \frac{X^{\varepsilon}(s)}{\varepsilon}, Y^{\varepsilon}(s)\right) d s .
\end{aligned}
$$


Comparing (3.3.15) and (3.3.16) we conclude ${ }^{\dagger}$ that

$$
\varlimsup_{\varepsilon \downarrow 0} P\left\{\sup _{0 \leq t \leq T}\left|X^{\varepsilon}(t)-\widetilde{X}^{\varepsilon}(t)\right|>\hat{o}\right\}=0, \quad \hat{o}>0 .
$$

Thus, it suffices to show that the processes $\widetilde{X}^{\varepsilon}(t)$ are (relatively) weakly compact in $D$.

We note first that

$$
\lim _{N \uparrow \infty} \sup _{0<\varepsilon<1} P\left\{\sup _{0 \leq t \leq T}\left|\widetilde{X}^{\varepsilon}(t)\right|>N\right\}=0
$$

making use of Kolmogorov's inequality for the martingale $M_{i}^{\varepsilon}(t)$ and (3. 3.10), (3.3.11). It suffices now $[19,20]$ to show that

$$
\begin{gathered}
E\left\{\left|\widetilde{X}^{\varepsilon}(t)-\widetilde{X}^{\varepsilon}(\sigma)\right|^{2}\left|\widetilde{X}^{\varepsilon}(\sigma)-\widetilde{X}^{\varepsilon}(s)\right|^{2}\right\} \leq C(t-s)^{2}, \\
0 \leq s \leq \sigma \leq t \leq T,
\end{gathered}
$$

where $C$ is a constant independent of $s, \sigma, t$ and $\varepsilon$. However, from (3. 3. 14) we have

$$
\text { (3.3.18) } \begin{aligned}
E\left\{\mid \widetilde{X}^{\varepsilon}(t)\right. & \left.-\left.\widetilde{X}^{\varepsilon}(s)\right|^{2} \mid \mathscr{F}_{s}\right\} \leq 4 E\left\{\left|\int_{s}^{t} b\left(X^{\varepsilon}(\sigma)\right) d \sigma\right|^{2} \mid \mathscr{F}_{s}\right\} \\
& +4 E\left\{\left|\int_{s}^{t} A\left(X^{\varepsilon}(\sigma), \frac{X^{\varepsilon}(\sigma)}{\varepsilon}, Y^{\varepsilon}(\sigma)\right) d \sigma\right|^{2} \mid \mathscr{I}_{s}\right\} \\
& +4 E\left\{\left|M^{\varepsilon}(t)-M^{\varepsilon}(s)\right|^{2} \mid \mathscr{F}_{s}\right\} \\
& \leq C(t-s), \quad C \text { a constant. }
\end{aligned}
$$

This clearly implies (3.3.17) and the first step (compactness in $D$ ) has been shown. Only the martingale estimate in (3.3.18) requires elaboration. We have

$$
\begin{aligned}
& E\left\{\mid M^{\varepsilon}(t)\right.\left.-\left.M^{\varepsilon}(s)\right|^{2} \mid \mathscr{F}_{s}\right\}=\sum_{i=1}^{n} E\left\{\left(M_{i}^{\varepsilon}(t)-M_{i}^{\varepsilon}(s)\right)^{2} \mid \mathscr{F}_{s}\right\} \\
&= \sum_{i=1}^{n} E\left\{\left\langle M_{i}^{\varepsilon}(t)\right\rangle-\left\langle M_{i}^{\varepsilon}(s)\right\rangle \mid \mathscr{F}_{s}\right\} \\
&=\sum_{i=1}^{n} E\left\{\int_{s}^{t} H_{i}\left(X^{\varepsilon}(\sigma), \frac{X^{\varepsilon}(\sigma)}{\varepsilon}, Y^{\varepsilon}(\sigma)\right) d \sigma \mid \mathscr{F}_{s}\right\} \\
& \leq C(t-s), \\
&+|x|^{2}=\sum_{i=1}^{n} x_{i .}^{2}
\end{aligned}
$$


the last being a consequence of the boundedness of $H_{i}, i=1,2, \cdots, n$.

Step 2, the identification of the limit, is an immediate consequence of (3. 3. 12) once we show that

(3. 3. 19) $\lim _{\varepsilon \downarrow 0} E\left\{\left|\int_{s}^{t} E\left\{A_{f}\left(X^{\varepsilon}(\sigma), \frac{X^{\varepsilon}(\sigma)}{\varepsilon}, Y^{\varepsilon}(\sigma)\right) \mid \mathscr{F}_{s}\right\} d \sigma\right|\right\}=0$

for each $f$ smooth and bounded. This is so because (3.3.12) and (3.3. 19) imply that for any limit measure of the processes $X^{\varepsilon}$, the expression

$$
f(X(t))-f(x)-\int_{0}^{t} \overline{\mathcal{L}} f(X(s)) d s
$$

is a martingale. Since the martingale problem associated with $\mathcal{L}$ has a unique solution the proof is complete.

To prove (3.3.19) it suffices to show that for each $x \in \boldsymbol{R}^{n}$ and uniformly in $y \in S$

(3. 3. 20) $\lim _{\varepsilon \downarrow 0} E_{x, y}\left\{\int_{0}^{t} A_{f}\left(X^{\varepsilon}(\sigma), \frac{X^{\varepsilon}(\sigma)}{\varepsilon}, Y^{\varepsilon}(\sigma)\right) d \sigma\right\}=0$

The function $A_{f}(x, \zeta, y)$ is bounded and continuous. If it were a smooth function of $x$ and $\zeta$ then (3.3.20) would follow immediately in view of (3.3.8) and the Fredholm alternative for $Q+F \cdot \frac{\partial}{\partial \zeta}$. That is, we would be able to construct a function $B_{f}(x, \zeta, y)$ (analogous to $u_{2}$ in (3. 1. 19)) such that

$$
Q_{x, \zeta} B_{f}+F(x, \zeta, y) \cdot \frac{\partial B_{f}}{\partial \zeta}+A_{f}=0
$$

and then apply (3.3.9) with $f^{\varepsilon}=\varepsilon^{2} B_{f}$.

Since $A_{f}$ is merely continuous in $x$ and $\zeta$ we first approximate it by a smooth function of $\zeta$ for each $x$ and $y$. Call it $A_{f}^{\alpha}$ so that

$$
\left|A_{f}(x, \zeta, y)-A_{f}^{\alpha}(x, \zeta, y)\right| \leq \alpha
$$

for all $(x, \zeta, y) \in \boldsymbol{R}^{n} \times T^{n} \times S$. Put

$$
\bar{A}_{f}^{\alpha}(x)=\int \widetilde{\bar{P}}(d \zeta d y ; x) A_{f}^{\alpha}(x, \zeta, y)
$$

The function $A_{f}^{\alpha}-\bar{A}_{f}^{\alpha}$ is smooth in $\zeta$ and satisfies (3.3.8). We now approximate this by a smooth function of $x$ uniformly on compact $x$-sets (without altering (3.3.8)) : 


$$
\left|A_{f}^{a}-\bar{A}_{f}^{a}-\tilde{A}_{f}^{a, \beta}\right| \leq \beta
$$

ve have

$$
\begin{aligned}
& \text { (3.3.25) |E\{ }\left\{\int_{0}^{t} A_{f} d \sigma\right\} \mid \\
& \leq\left|E\left\{\int_{0}^{t}\left(A_{j}-A_{f}^{a}\right) d \sigma\right\}\right|+\left|E\left\{\int_{0}^{t}\left(A_{f}^{a}-\bar{A}_{f}^{a}-\tilde{A}_{f}^{\alpha, \beta}\right) d \sigma\right\}\right| \\
& \quad+\left|E\left\{\int_{0}^{t} \bar{A}_{f}^{a} d \sigma\right\}\right|+\left|E\left\{\int_{0}^{t} \widetilde{A}_{f}^{\alpha, \beta} d \sigma\right\}\right| .
\end{aligned}
$$

The first and third terms on the right side of (3.3.25) are small if $\alpha$ is small because of (3.3.22) and the fact that $\bar{A}_{f}^{a} \rightarrow 0$ as $\alpha \rightarrow 0$ by (3.3.8). With $\alpha$ fixed the second term on the right in (3.3.25) is small for $\beta$ small because of (3.3.24) and the fact that $X^{\varepsilon}(t), 0 \leq t \leq T$, stays in a compact set uniformly in $\varepsilon$ with high probability (compactness). With $\alpha$ and $\beta$ fixed the last term on the right side of (3.3.25) goes to zero as $\varepsilon \rightarrow 0$ because $\tilde{A}_{f}^{\alpha, \beta}$ is smooth and (3.3.8) holds for it. The proof of the theorem is complete.

\section{4. Boundary Layer Coordinates}

In the next sections we shall be concerned with boundary value problems associated with $\mathcal{L}^{s}$ of (3.3.1) without $\frac{x}{\hat{\varepsilon}}$ dependence, i.e.,

$$
\mathcal{L}^{\varepsilon}=\frac{1}{\varepsilon^{2}} Q_{x}+\frac{1}{\varepsilon} F(x, y) \cdot \frac{\partial}{\partial x}+G(x, y) \cdot \frac{\partial}{\partial x}
$$

where $Q_{x}$ is defined as in (3.1.2) but without " dependence. It will be necessary to express the operator $\mathcal{L}^{\varepsilon}$ in convenient local coordinates near the boundaries which are always in $x$-space. We do this in this section.

Let $\mathscr{D}$ be a bounded domain in $\mathbb{R}^{n}$ and $\partial \mathscr{D}$ denote its boundary which we assume is a smonth $(n-1)$-dimensional hypersurface. Since the surface $\partial \mathscr{D}$ is a compact subset of $\mathbb{R}^{n}$ it can be covered by a finite number of open sets that include a $\delta$ neighborhood of the boundary (i.e., $\left.\bigcup_{x \in \partial \mathscr{D}} S(x, \hat{0}), S(x, \delta)=\{y:|x-y|<\delta\}\right)$. These finitely many open sets will be referred to as coordinate patches.

In each coordinate patch the surface is given parametrically by $\tilde{\xi}$ $=\xi(\gamma)$, $\xi$ a vector in $\mathbb{R}^{n}$ and $\gamma=\left(\gamma_{1}, \cdots, \gamma_{n-1}\right)$ is the parameter point that 
runs over a subset of $\mathbb{R}^{n-1}$ with compact closure. We shall confine attention to a single coordinate patch.

We introduce new coordinates, the stretched coordinates, $x \rightarrow(\gamma, \varepsilon \eta)$ given by $^{\dagger}$

$$
x=\xi(\gamma)+\varepsilon \eta \hat{n}(\gamma), \quad \eta=\frac{(x-\xi(x)) \cdot \hat{n}(x)}{\varepsilon},
$$

where for each $x$ in the patch $\xi(\gamma)$ is the (unique since $x$ is near enough to the surface) point on the surface such that $\xi(\gamma)-x$ and $\hat{n}(\gamma)$, the unit outer normal at $\xi(\gamma)$, are parallel. The parameter $\gamma$ runs over a subset of $\mathbb{R}^{n-1}$ and $\eta \leq 0$ (the lower bound of $\eta$ goes to $-\infty$ like $1 / \varepsilon$ ).

We assume that $\left(\gamma_{1}, \gamma_{2}, \cdots, \gamma_{n-1}\right)$ are orthogonal curvilinear coordinates for $\xi(\gamma)$ that is

$$
a^{i}=a^{i}(\gamma)=\frac{1}{\alpha_{i}(\gamma)} \frac{\partial \xi(\gamma)}{\partial \gamma_{i}} \quad i=1,2, \cdots, n-1
$$

are orthonormal vectors that span the tangent space of the surface at $\xi(\gamma)$. The functions $\alpha_{i}(\gamma), i=1, \cdots, n-1$ are the scale factors of the local coordinates on the surface. Note that $\left(a^{1}, a^{2}, \cdots, a^{n-1}, \hat{n}\right)$ is an orthonormal frame in $\mathbb{R}^{n}$ at each point of the surface. Define also the curvature matrix

$$
\rho(\gamma)=\left(\rho_{i j}(\gamma)\right)=\left(\hat{n}(\gamma) \cdot \frac{\partial^{2} \xi(\gamma)}{\partial \gamma_{i} \partial \gamma_{j}}\right) \quad i, j=1,2, \cdots, n-1
$$

and write

$$
\alpha(\gamma)=\left(\alpha_{i j}(\gamma)\right)=\left(\alpha_{i}(\gamma) \delta_{i j}\right) \quad i, j=1,2, \cdots, n-1
$$

$$
J^{\mathcal{E}}(\gamma, \eta)=\left(I-\varepsilon \eta \alpha^{-2} \rho\right)^{-1} \alpha^{-1}, \quad I=(n-1) \times(n-1) \text { identity. }
$$

The elements of this $(n-1) \times(n-1)$ matrix are denoted by $J_{i j}^{\varepsilon}$.

It is easily seen that in the new coordinates $(\gamma, \varepsilon \eta), x$-derivatives are expressed as follows

$$
\frac{\partial}{\partial x_{p}}=\frac{1}{\varepsilon} \hat{n}_{p}(\gamma) \frac{\partial}{\partial \eta}+\sum_{i, j=1}^{n-1} a_{p}^{i}(\gamma) J_{i j}^{\varepsilon}(\gamma, \eta) \frac{\partial}{\partial \gamma_{j}}, \quad p=1,2, \cdots, n .
$$

Here $\hat{n}_{p}$ and $a_{p}^{i}$ are the $p^{\iota h}$ Cartesian coordinates of the vectors $\hat{n}$ and

\footnotetext{
† We shall abuse notation by writing $\xi(x)$ and $\hat{n}(x)$ instead of $\xi(\gamma(x))$ and $\hat{n}(\gamma(x))$ when we want to return from $(\gamma, \varepsilon \eta)$ to $x$.
} 
$a^{i} i=1,2, \cdots, n-1$. The operator $\mathcal{L}^{\varepsilon}$ of (3. 4. 1) takes the following form in terms of the boundary layer coordinates $(\gamma, \varepsilon \eta)$.

$$
\begin{aligned}
\mathcal{L}^{\varepsilon}=\mathcal{L}_{B L}^{\varepsilon}= & \frac{1}{\varepsilon^{2}} Q_{\xi+\varepsilon \eta \hat{n}} \\
& +\sum_{p=1}^{n}\left[\frac{1}{\varepsilon} F_{p}(\xi+\varepsilon \eta \hat{n}, y)+G_{p}(\xi+\varepsilon \eta \hat{n}, y)\right] \\
& \cdot\left[\frac{1}{\varepsilon} \hat{n}_{p} \frac{\partial}{\partial \eta}+\sum_{i, j=1}^{n-1} a_{p}^{i} J_{i j}^{\varepsilon} \frac{\partial}{\partial \gamma_{j}}\right] .
\end{aligned}
$$

From (3.4.8) we see that

$$
\mathcal{L}_{B L}^{\varepsilon}=\frac{1}{\varepsilon^{2}} \mathcal{L}_{B L 1}+\frac{1}{\varepsilon} \mathcal{L}_{B L 2}+\mathcal{L}_{B L 3}+\mathcal{L}_{B L 4}^{\varepsilon^{\prime}}
$$

where $\mathcal{L}_{B L 4}^{\varepsilon}$ is defined by (3.4.9) and has coefficients proportional to $\varepsilon$. Comparing (3.4.8) and (3.4.9) we obtain the following expression for the operators $\mathcal{L}_{B L 1}, \mathcal{L}_{\boldsymbol{B L 2}}$ and $\mathcal{L}_{\boldsymbol{B L} 3}$ in (3.4.9).

$$
\mathcal{L}_{B L 1}=Q_{\xi(\tau)}+F(\hat{\xi}(\hat{\imath}), y) \cdot \hat{n}(\gamma) \frac{\partial}{\partial \eta}
$$

$$
\begin{aligned}
\mathcal{L}_{B L 2}=\sum_{p=1}^{n} & {\left[\eta \hat{n}_{p}(\gamma) Q_{\xi(\gamma)}^{p}+G_{p}(\xi(\gamma), y) \hat{n}_{p}(\gamma) \frac{\partial}{\partial \eta}\right.} \\
& +\sum_{q=1}^{n} \eta F_{p, q}(\xi(\gamma), y) \hat{n}_{q}(\hat{\imath}) \hat{n}_{p}(\gamma) \frac{\partial}{\partial \eta} \\
& \left.+\sum_{i=1}^{n-1} F_{p}(\xi(\gamma), y) a_{p}^{i}(\gamma) \frac{1}{\alpha_{i}(\gamma)} \frac{\partial}{\partial \gamma_{i}}\right]
\end{aligned}
$$

$$
\begin{aligned}
\mathcal{L}_{B L 3}=\sum_{p, q=1}^{n} & {\left[\frac{1}{2} \eta^{2} \hat{n}_{p}(\gamma) \hat{n}_{q}(\gamma) Q_{\xi(\gamma)}^{p q}\right.} \\
& +\frac{\eta^{2}}{2} \sum_{r=1}^{n} F_{p, q r}(\dot{\xi}(\tilde{\imath}), y) \hat{n}_{q}(\tilde{\imath}) \hat{n}_{p}(\gamma) \hat{n}_{r}(\gamma) \frac{\partial}{\partial \eta} \\
& +\eta G_{p, q}(\xi(\hat{\imath}), y) \hat{n}_{q}(\gamma) \hat{n}_{p}(\gamma) \frac{\partial}{\partial \eta} \\
& \left.+\eta^{2} \sum_{i, j=1}^{n-1} F_{p, q}(\xi(\gamma), y) \hat{n}_{q}(\gamma) a_{q}^{i}(\tilde{\gamma}) \frac{\rho_{i j}(\gamma)}{\alpha_{j}(\gamma) \alpha_{i}^{2}(\gamma)} \frac{\partial}{\partial \gamma_{j}}\right] \\
& +\sum_{p=1}^{n} G_{p}(\xi(\gamma), y) a_{p}^{i}(\tilde{\gamma}) \frac{1}{\alpha_{i}(\gamma)} \frac{\partial}{\partial \gamma_{i}} \cdot
\end{aligned}
$$


Here $Q_{\xi}^{p}$ denotes the operator $Q$ of (3.1.2) (without $\zeta$ dependence) with its coefficients differentiated with respect to $x_{p}, Q_{\xi}^{p q}$ denotes differentiation of the coefficients with respect to $x_{p}$ and $x_{q}$ and $F_{p, q}$, etc., denotes the derivative of $F_{p}$ with respect to $x_{q}$.

\section{5. Asymptotic Expansions for Absorbing Boundary Conditions (without cells)}

In this section we shall analyze the asymptotic behavior as $\varepsilon \rightarrow 0$ of the following problem. Let $\mathscr{D} \subset \mathbb{R}^{n}$ be a bounded open set with smooth boundary $\partial \mathscr{D}$. Let $u^{\varepsilon}(x, y)$ satisfy the boundary value problem

$$
\mathcal{L}^{\varepsilon} u^{\varepsilon}(x, y)+f(x, y)=0, \quad\{x \in \mathscr{D}, y \in S\} \cup\left\{x \in \partial \mathscr{D}, y \in S^{-}\right\},
$$

$$
u^{\varepsilon}(x, y)=g(x, y), \quad x \in \partial \mathscr{D}, y \in S^{+} \text {, }
$$

$$
\mathcal{L}^{\varepsilon}=\frac{1}{\varepsilon^{2}} Q_{x}+\frac{1}{\varepsilon} F(x, y) \cdot \frac{\partial}{\partial x}+a(x, y), \quad a(x, y) \leq 0,
$$

$$
S^{+}=S^{+}(x)=\{y \in S: F(x, y) \cdot \hat{n}(x)>0, \quad x \in \partial \mathscr{D}\} \text {. }
$$

Here $\hat{n}(x)$ is the unit outer normal at $x \in \partial \mathscr{D}, f, g$ and a are smooth functions on $\mathbb{R}^{n} \times S, F(x, y)$ is a smooth $n$-vector function on $\mathbb{R}^{n} \times S$ ( $S$ is a compact metric space as in Section 3.1; for example the unit ball in $\mathbb{R}^{n}$ ) and $Q_{x}$ is given by

$$
Q_{x} f(y)=q(x, y) \int \pi(x, y, d z) f(z)-q(x, y) f(y) .
$$

We assume that $q$ and $\pi$ depend smoothly on $x$, that they are continuous in $y$ and that (2.5.3) and (2.5.4) hold (uniformly in $x$ ) so that the Fredholm alternative holds for $Q_{x}$ in the form (2.5.9), (2.5.10). Note that in (3.5.3) the term $G \cdot \frac{\partial}{\partial x}$ (cf. (3.1.1)) has been dropped for simplicity; otherwise $S^{+}$depends on $\varepsilon$. Also we focus attention on the time-independent, steady, problem (3.5.1) in order to avoid problems with initial-boundary layers, i.e., difficulties near space-time corners where special considerations are necessary in constructing complete expansions.

The weak convergence result of Section 3. 3 handles the stopped process $X^{\varepsilon}\left(t \wedge \tau^{\varepsilon}\right), \tau^{\varepsilon}=$ first exit time of $X^{\varepsilon}(t)$ from $\mathscr{D}$, under appropriate conditions. We seek here to analyze, in addition and in more detail, the 
behavior of $Y^{\varepsilon}(t)$ near $\partial \mathscr{D}$ i.e., boundary layer phenomena. That is why we focus attention on (3.5.1), (3.5.2). We shall not consider here the analogous problem with cellular structure.

We begin by looking for an expansion of the form

(3. 5.6) $\quad u^{\varepsilon}(x, y)$

$$
=\sum_{k=0}^{\infty} \varepsilon^{k} u_{k}(x, y)+\left.\sum_{k=0}^{\infty} \varepsilon^{k} u_{k}^{B L}(\eta, \xi(x), y)\right|_{\eta=((x-\xi(x)) \cdot \hat{n}(x)) / \varepsilon}
$$

where $u_{k}^{B L}$ are the boundary layer terms and they are functions of the local boundary layer coordinates (cf. Section 3.4). We assume that outside a fixed neighborhood of the boundary $u_{k}^{B L} \equiv 0, k=0,1,2, \cdots$. Since the boundary layer terms, it iurns out, decay exponentially away from the boundary this is easily accomplished by a smooth truncation. On the other hand it is convenient to have $\iota_{k}^{B L}(\eta, \xi(\gamma), y)$ defined for all $\eta \leq 0$ and so we shall allow this mild inconsistency in the following to avoid complicating the formulas with cutoffs.

The construction of the expansion is as follows. In the interior of $D$ we require that

$$
\mathcal{L}^{\varepsilon}\left(\sum_{k=0}^{\infty} \varepsilon^{k} u_{k}\right)+f=0
$$

This yields the following problems.

$$
\begin{aligned}
\mathcal{L}_{1} u_{0} & =0 \\
\mathcal{L}_{1} u_{1}+\mathcal{L}_{2} u_{0} & =0 \\
\mathcal{L}_{1} u_{2}+\mathcal{L}_{2} u_{1}+\mathcal{L}_{3} u_{0}+f & =0 \\
\mathcal{L}_{1} u_{n}+\mathcal{L}_{2} u_{n-1}+\mathcal{L}_{3} u_{n-2} & =0, \quad n=3,4,5, \cdots .
\end{aligned}
$$

Here we have

$$
\mathcal{L}_{1}=Q_{x}, \quad \mathcal{L}_{2}=F(x, y) \cdot \frac{\partial}{\partial x}, \quad \mathcal{L}_{3}=a(x, y) .
$$

From the ergodic properties of $\mathcal{L}_{1}$ it follows that $u_{0}=u_{0}(x)$ i.e., it is independent of $v$ and the first equation in (3.5.7) is satisfied.

Let $\bar{P}(A ; x), A \subset S$ be the invariant measure associated with the process whose generator is $Q_{x}$ of (3.5.5) ( $x$ is a parameter). Define, as in (2.5.8), the potential kernel 


$$
\psi(y, A ; x)=\int_{0}^{\infty}[P(t, y, A ; x)-\bar{P}(A ; x)] d t,
$$

where $P(t, y, A ; x)$ is the transition function. In order to solve the second equation in (3.5.7) we assume (as in (3.1.13)) that

$$
\int_{S} F(x, y) \bar{P}(d y ; x) \equiv 0 \text {. }
$$

Then, by the Fredholm alternative

$$
u_{1}=u_{10}-\Upsilon_{1}^{-1} \mathcal{L}_{2} u_{0}
$$

or

$$
u_{1}(x, y)=u_{10}(x)+\int_{S} \psi(y, d z ; x) F(x, z) \cdot \frac{\partial u_{0}(x)}{\partial x}
$$

As in Section 3. 1, $u_{0}(x)$ and $u_{10}(x)$, etc., are determined from solvability conditions for $u_{2}$ and $u_{3}$ etc., in (3.5.7).

Substituting (3.5.11) into the third equation (3.5.7) we obtain

$$
\mathcal{L}_{1} u_{2}+\mathcal{L}_{2}\left(u_{10}-\mathcal{L}_{1}^{-1} \mathcal{L}_{2} u_{0}\right)+\mathcal{L}_{3} u_{0}+f=0
$$

and therefore the solvability condition for (3.5.13) yields the following equation for $\iota_{0}(x)$.

$$
\bar{L} u_{0}(x)+\bar{f}(x)=0, \quad x \in \mathscr{D},
$$

where

$$
\begin{aligned}
& \overline{\mathcal{L}} h(x)= \int_{s} \int_{S} \bar{P}(d y ; x) F(x, y) \\
& \times \frac{\partial}{\partial x}\left[\psi(y, d z ; x) F(x, z) \cdot \frac{\partial h(x)}{\partial x}\right] \\
&+\int_{S} \bar{P}(d y ; x) a(x, y) h(x) \\
& \bar{f}(x)=\int_{S} \bar{P}(d y ; x) f(x, y) .
\end{aligned}
$$

Boundary conditions for $\iota_{0}(x)$ are obtained from the boundary layer expansion later on. Note that $u_{10}$ drops out of the solvability condition for (3.5.13) in view of (3.5.10).

It is easily seen, exactly as in Section 3.1 , that $\iota_{10}(x)$ satisfies an 
equation of the form (3.5.14) also. Now the inhomogeneous term depends on $u_{0}(x)$ however. So, up to boundary conditions, $u u_{0}(x)$ and $u_{10}(x)$, hence $u_{1}(x, y)$, are determined. The higher order terms go the same way.

Let us next consider the boundary layer terms (3.5.6). We employ the notation of Section 3.4 systematically. It is required that

$$
\begin{aligned}
& \mathcal{L}_{B L}^{\varepsilon}\left(\sum_{k=0}^{\infty} \varepsilon^{k} u_{k}^{B L}\right)=0, \\
& u_{k}^{B L} \rightarrow 0 \text { as } \eta \rightarrow-\infty, \quad k=0,1,2, \cdots,
\end{aligned}
$$

and

$$
\left.\left(u_{0}+u_{0}^{B L}\right)\right|_{\substack{x \in \partial \mathscr{D} \\ y \in S^{+}}}=g,
$$

$$
\left.\left(u_{k}+u_{k}^{B L}\right)\right|_{\substack{x \in \partial \mathscr{D} \\ y \in S^{+}}}=0, \quad k=1,2, \cdots
$$

Since $\mathcal{L}_{B L}^{\varepsilon}$ in (3.4.9) stops with $\mathcal{L}_{B L 4}^{\varepsilon}$ we shall stop here with $u_{2}^{B L}$; further computations require expanding $\mathcal{L}_{B L 4}^{s}$ and they are not particularly revealing. Now from (3.5.17) and (3.4.9) it follows that

$$
\begin{gathered}
\mathcal{L}_{B L 1} u_{0}^{B L}=0 \\
\mathcal{L}_{B L 1} u_{1}^{B L}+\mathcal{L}_{B L 2} u_{0}^{B L}=0 \\
\mathcal{L}_{B L 1} u_{2}^{B L}+\mathcal{L}_{B L 2} u_{1}^{B L}+\mathcal{L}_{B L 3} u_{0}^{B L}=0
\end{gathered}
$$

Let us look in detail at the determination of $u_{0}^{B L}$. We have that, with $\gamma$ a parameter (or $\xi=\xi(x), x$ a parameter)

$(3.5 .24) \quad\left(Q_{\xi(r)}+F(\xi(\gamma), y) \cdot \hat{n}(\gamma) \frac{\partial}{\partial \eta}\right) u_{0}^{B L}(\eta, \xi(\gamma), y)=0, \quad \eta<0$

$$
u_{0}^{B L}(0, \xi(\gamma), y)=g(\xi(\gamma), y)-u_{0}(\xi(\gamma)), \quad y \in S^{+}
$$

$$
\lim _{\eta \rightarrow-\infty} u_{0}^{B L}(\eta, \xi(\gamma), y)=0 \text {. }
$$

This is exactly the problem we analyzed in Section 2. 6, specifically, equation (2.6.12). In order that (3.5.24)-(3.5.26) have a solution, which will be unique, it is sufficient that

$$
\int_{S^{+}} \bar{U}(d y ; \hat{\xi}(\gamma))\left(g(\xi(\gamma), y)-u_{0}(\xi(\gamma))\right)=0
$$


where $\bar{U}$ is the invariant measure of the transmission operator (cf. (2.6. 15)) and now depends parametrically on the boundary point $\xi(r)$. It follows that the correct boundary condition for $u_{0}(x), x \in \partial \mathscr{D}$ is

$$
\left.u_{0}(x)\right|_{x \in \partial \mathscr{D}}=\left.\int_{S^{+}} \bar{U}(d y ; x) g(x, y)\right|_{x \in \partial \mathscr{D}} .
$$

Equation (3.5.14) and boundary condition (3.5.27) determine $u_{0}(x)$ and then (3. 5.24)-(3.5.26) determines $u_{0}^{B L}$. Thus, the first term in the expansion (3.5.6) is completely determined.

To solve the equation (3.5.22) so that (3.5.18) holds for $u_{2}^{B L}$ we use Theorem 2 of Section 2.7, which was obtained expressly for the present situation. The quantity $\mathcal{L}_{B L_{2}} \iota_{0}^{B L}$ is exponentially decaying as $\eta \rightarrow$ $-\infty$ and if $\iota_{10}$ is chosen appropriately at the boundary $\partial \mathscr{D}$ (cf. (2.7.41)) then, $u_{1}(x, y)$ and $u_{1}^{B L}(\eta, \xi(\gamma), y)$ are uniquely determined. Identical considerations lead to the determination of $\iota_{2}$ and $u_{2}^{B L}$.

Theorem. Assume that $F, Q, f, g, a \leq 0$, depend smoothly on $x \in \overline{\mathscr{D}}$ and continuously on $y \in S$, that $\partial \mathscr{D}$ is smooth and that $\overline{\mathcal{L}}$ of (3.5.15) is uniformly elliptic in $\bar{D}$. Assume that $Q$ satisfies hypotheses such as (2.5.3) and (2.5.4) (uniformly in $x$ ) so that it is ergodic. Assume that (3.5.10) holds. Then the solution $u^{\varepsilon}(x, y)$ of (3.5.1), (3.5.2) satisfies ${ }^{+}$

$$
\begin{gathered}
(3.5 .28) \sup _{x \in \bar{D}} \sup _{y \in S}\left|u^{\varepsilon}(x, y)-u_{0}(x)-u_{0}^{B L}\left(\frac{(x-\hat{\xi}(x)) \cdot \hat{n}(x)}{\varepsilon}, \hat{\xi}(x), y\right)\right| \\
=O(\varepsilon) .
\end{gathered}
$$

Proof. First of all since $\overline{\mathcal{L}}$ is uniformly elliptic and everything is smooth so is $u_{0}(x)$, the solution of (3.5.14) and (3.5.27). All other quantities that figure in the above construction are also smooth.

Let

$$
\begin{aligned}
W^{\varepsilon}\left(x, y^{\prime}\right)= & u^{\varepsilon}(x, y)-\left(u u_{0}(x)+\varepsilon u_{1}(x, y)+\varepsilon^{2} u_{2}(x, y)\right) \\
& -\left(u_{0}^{B L}(\eta, \xi(x), y)+\varepsilon u_{1}^{B L}\left(\eta, \xi(x), y^{\prime}\right)\right. \\
& \left.+\varepsilon^{2} u_{2}^{B L}(\eta, \xi(x), y)\right)\left.\right|_{\eta=((x-\xi(x)) \cdot \hat{n}(x)) / c} .
\end{aligned}
$$

\footnotetext{
' Recall the convention about cutoffs for $u_{0}^{B L}$ stated below (3.5.6).
} 
By construction we have that

$$
\begin{gathered}
\mathcal{L}^{\varepsilon} W^{\varepsilon}(x, y)=O(\varepsilon) \text { in }\{x \in \mathscr{D}, y \in S\} \cup\left\{x \in \partial \mathscr{D}, y^{\prime} \in S^{-}\right\} \\
W^{\varepsilon}\left(x, y^{\prime}\right)=0, x \in \partial \mathscr{D}, y \in S^{\prime} .
\end{gathered}
$$

Thus, if $a\left(x, y^{\prime}\right) \leq-\delta<0$ then (3.5.28) follows immediately from (3.5.30) and (3.5.29). If on the other hand we have

$$
E_{x}\left\{\tau^{\varepsilon}\right\} \leq c<\infty
$$

where

$$
\tilde{r}^{s}=\inf \left\{t \geq 0: X^{\varepsilon}(t) \notin \overline{\mathscr{D}}\right\}
$$

then again the result follows. Here $X^{\varepsilon}(t)$ has generator $\varepsilon^{-2} Q_{x}+\varepsilon^{-1} F$ $\cdot \frac{\partial}{\partial x}$. The proof is complete in riew of the following.

Lemma. Estimate (3.5.31) is true under, in fact, weaker hypotheses than the present ones, provided $\varepsilon$ is sufficiently' small.

Proof of the Lemma. Let $X(t)$ denote the limit process with generator (cf. (3.5.15))

$$
\begin{aligned}
\bar{L} h(x)= & \int_{S} \int_{S} \bar{P}(d y ; x) F(x, y) \\
& \cdot \frac{\partial}{\partial x}\left(\psi(y, d \approx ; x) F(x, \approx) \cdot \frac{\partial h(x)}{\partial x}\right) \\
\equiv & \frac{1}{2} \sum_{i, j=1}^{n} \bar{a}_{i j}(x) \frac{\partial^{2} h(x)}{\partial x_{i} \partial x_{j}}+\sum_{j=1}^{n} \bar{b}_{j}(x) \frac{\partial h(x)}{\partial x_{j}}
\end{aligned}
$$

Let $\tau$ be the first exit time of $X(t)$ from $\mathscr{D}$. From the uniform ellipticity of $\overline{\mathcal{L}}$ (which is an unnecessarily strong hypothesis) it follows that there is a function $Y(x), x \in \bar{D}$, bounded smooth and positive such that

$$
\overline{\mathcal{L}} V(x) \leq-1
$$

But then

$$
\begin{aligned}
0 & \leq E_{x}\{V(X(\tau \wedge t))\} \\
& =V^{\top}(x)+E_{x} \int_{0}^{\tau, \backslash t} \overline{\mathcal{L}} \Gamma^{\top}(X(s)) d s \leq \Gamma^{\top}(x)-E_{x}\{\tau \wedge t\}
\end{aligned}
$$


or

$$
E_{x}\{\tau \wedge t\} \leq V(x)
$$

and hence, letting $t \uparrow \infty$,

$$
E_{x}\{\tau\} \leq \sup _{x \in \overline{\mathscr{D}}} V(x)=c<\infty
$$

For example, we may take $V(x)=K-e^{\alpha x_{1}}$ with $K$ and $\alpha$ sufficiently large.

With $T^{\prime}(x)$ so defined, define $V_{1}(x, y)$ and $V_{2}(x, y)$ by

$$
\begin{gathered}
Q_{x} V_{1}(x, y)+F(x, y) \cdot \frac{\partial V(x)}{\partial x}=0 \\
Q_{x} V_{2}(x, y)+F(x, y) \cdot \frac{\partial V_{1}(x, y)}{\partial x}-\overline{\mathcal{L}} V(x)=0 .
\end{gathered}
$$

Clearly $V_{1}$ and $V_{2}$ are well defined bounded and smooth. Furthermore

$$
\left(\frac{1}{\varepsilon^{2}} Q_{x}+\frac{1}{\varepsilon} F \cdot \frac{\partial}{\partial x}\right)\left(V+\varepsilon V_{1}+\varepsilon^{2} V_{2}\right)=\overline{\mathcal{L}} V+\varepsilon F \cdot \frac{\partial V_{2}}{\partial x} .
$$

It follows that for $\varepsilon$ sufficiently small $V^{\varepsilon}=V+\varepsilon V_{1}+\varepsilon^{2} V_{2}$ is nonnegative and, say,

$$
\left(\frac{1}{\varepsilon^{2}} Q_{x}+\frac{1}{\varepsilon} F \cdot \frac{\partial}{\partial x}\right) V^{\varepsilon} \leq-\frac{1}{2}, \quad x \in \bar{D}, y \in S .
$$

This implies (3.5.31) the same way (3.5.34) implied (3.5.35). The proof is complete.

\section{6. Asymptotic Expansion for Reflecting Boundary Conditions (without cells)}

In this section we shall analyze the asymptotic behavior as $\varepsilon \rightarrow 0$ of the following problem. Let $\mathscr{D} \subset \boldsymbol{R}^{n}$ be a bounded open set with smooth boundary $\partial \mathscr{D}$. Let $u^{\varepsilon}(x, y)$ satisfy the problem (cf. (2.3.4)).

$$
\begin{gathered}
\mathcal{L}^{\varepsilon} u^{\varepsilon}(x, y)+f(x, y)=0, \quad x \in \mathscr{D}, y \in S ; x \in \partial \mathscr{D}, y \in S^{-}, \\
\int_{S^{-}} B(x, y, d z) u^{\varepsilon}(x, z)-u^{\varepsilon}(x, y)+\varepsilon b(x, y) u^{\varepsilon}(x, y) \\
+\varepsilon g(x, y)=0, \quad x \in \partial \mathscr{D}, y \in S^{+},
\end{gathered}
$$




$$
\mathcal{L}^{\varepsilon}=\frac{1}{\varepsilon^{2}} Q_{x}+\frac{1}{\varepsilon} F(x, y) \cdot \frac{\partial}{\partial x}+a(x, y),
$$

$$
S^{ \pm}=S^{\lrcorner}(x)=\{y \in S: F(x, y) \cdot \hat{n}(x) \geqq 0, x \in \partial \mathscr{D}\},
$$

$$
a(x, y) \leq-\alpha<0, b(x, y) \leq 0 \text {. }
$$

As in Section 3.5 we do not have $\frac{x}{\varepsilon}$ dependence, $Q_{x}$ is as defined in (3. 5.5), $\hat{n}(x)$ is the unit outer normal to $\partial \mathscr{D}, f, g, a$ and $b$ are smooth functions on $\boldsymbol{R}^{n} \times S$ and $F$ is a smooth n-vector function on $\boldsymbol{R}^{n} \times S$ such that

$$
\int_{S} F(x, y) \bar{P}(d y ; x) \equiv 0
$$

Here $\bar{P}(A ; x), A \subset S$, is the invariant measure of the process associated with $Q_{x}$; we assume as in 3.5 that it is ergodic and the Fredholm alternative holds in the form (2.5.9) and (2.5.10). Note that $g$ is multiplied by $\varepsilon$ in (3.6.2). This is the appropriate scaling so that $u^{\varepsilon}=O(1)$ as $\varepsilon \rightarrow 0$; otherwise if $g=O(1)$, then $u^{\varepsilon}=O\left(\varepsilon^{-1}\right)$.

We need the following assumptions on the boundary operator $B$ (cf. (2.2.9) and (2.6.31)).

(3. 6. 7) $B\left(x, y^{\prime}, A\right), x \in \partial \mathscr{D}, y \in S^{\lrcorner}, A \subset S^{-}$depends $^{\dagger}$ smoothly on $x$, is continuous in $y$ and given $\delta_{1}>0$ there is a $\delta_{2}>0$ such that

$$
\inf _{x \in \partial \subseteq \mathcal{D}} \inf _{y \in S^{+}} \int_{\hat{n} \cdot F<-\hat{o}_{1}} B(x, y, d z) \geq \hat{o}_{2} .
$$

(3.6.8) There is a nontrivial reference probability measure $\widetilde{\phi}$ on $S^{-}$ and a $\delta_{3}>0$ such that $\widetilde{\phi}(A)>0$ if $B(x, y, A)=1$ and $B(x, y, A) \geq \delta_{3} \widetilde{\phi}(A)$ for all $x \in \partial \mathscr{D}, y \in S^{+}, A \subset S^{-}$.

Under the above hypotheses (some of them are needed only for the asymptotics) (3.6.1) and (3.6.2) have a unique solution. Notice the necessity of having (3.6.5) here; the problem with $a=b=0$ does not have a solution for general $f$ and $g$.

We begin by looking for an expansion of the form

$$
u^{\varepsilon}(x, y)=\sum_{k=0}^{\infty} \varepsilon^{k} u_{l}(x, y)+\left.\sum_{k=n}^{\infty} \varepsilon^{k} u_{k}^{B L}(\eta, \xi(x), y)\right|_{\eta=((x-\xi(x)) \cdot \hat{n}(x)) / \varepsilon}
$$

\footnotetext{
'That is, $B\left(x, y, A \cap S^{-}\right), A \subset S$, is smooth in $x$.
} 
where $u_{k}^{B L}$ are the boundary layer terms and they are functions of the local boundary coordinates (cf. Section 3.4). We assume as in 3.5 that they vanish outside a fixed neighborhood of $\partial \mathscr{D}$. On the other hand it will be convenient to disregard $\mathrm{d}^{\dagger}$ this in the computation and suppose that they are defined for all $\eta \leq 0$; it turns out they decay to zero exponentially fast as $\eta \rightarrow-\infty$.

In the interior, the expansion goes exactly as it did in Section 3.5, i.e., we have (3.5.7)-(3.5.16). In particular, $\iota_{0}(x)$ satisfies (3.5.14) with $\overline{\mathcal{L}}$ and $\bar{f}$ given by (3.5.15) and (3.5.16).

Let us consider the boundary layer terms in (3.6.9). It is required that

$$
\begin{gathered}
\mathcal{L}_{B L}^{c}\left(\sum_{k=0}^{\infty} \varepsilon^{k} u_{k}^{B L}\right)=0, \\
u_{k}^{B L} \rightarrow 0 \text { as } \eta \rightarrow-\infty, \quad k=0,1,2, \cdots, \\
\int_{S^{-}} B\left(u_{k}+u_{k}^{B L}\right)-\left(u_{k}+u_{k}^{B L}\right)+b\left(u_{k-1}+u_{k-1}^{B L}\right)=0, \\
k \neq 1, x \in \partial \mathcal{D}, y \in S^{+}, \\
\int_{S^{-}} B\left(u_{1}+u_{1}^{B L}\right)-\left(u_{1}+u_{1}^{B L}\right)+b\left(u_{0}+u_{0}^{B L}\right)+g=0 .
\end{gathered}
$$

The operator $\mathcal{L}_{\boldsymbol{B} L}^{\varepsilon}$ is given by (3.4.9) and, as in 3.5, we shall not expand it beyond $\mathcal{L}_{B L 3}$ so that we stop here with the term $u_{2}^{B L}$. We have clearly that

$$
\begin{aligned}
& \mathcal{L}_{B L 1} u_{0}^{B L}=0 \\
& \mathcal{L}_{B L_{1}} u_{1}^{B L}+\mathcal{L}_{B L 2} u_{0}^{B L}=0 \\
& \mathcal{L}_{B L_{1}} u_{2}^{B L}+\mathcal{L}_{B L_{2}} u_{1}^{B L}+\mathcal{L}_{B L_{3}} u_{0}^{B L}=0 .
\end{aligned}
$$

From (3.6.14), (3.6.11) with $k=0$, and (3.6.12) with $k=0$ we conclude that*

$$
u_{0}^{B L} \equiv 0
$$

Thus, $u_{1}^{B L}(\eta, \xi(x), y)$ satisfies the problem $(\xi(x)$ a parameter $)$

We adopt again the convention about cutoffs stated below (3.5.6).

* This means that boundary layer terms are $O(\varepsilon)$ and should be contrasted with the absorbing case (Section 3.5) where they are $O(1)$. 


$$
\begin{aligned}
& \mathcal{L}_{B L_{1}} u_{1}^{B L}=0, \quad \eta<0, \\
& u_{1}^{B L} \rightarrow 0 \quad \text { as } \quad \eta \rightarrow-\infty \text {, } \\
& \int_{S^{-}} B u_{1}^{B L}-u_{1}^{B L}=-g-\int_{S^{-}} B u_{1}+u_{1}-b u_{0}, \\
& \eta=0, v \in S^{-} \text {. }
\end{aligned}
$$

But this is exactly problem (2.6.26) (with $\xi(x)$, the point on the boundary, a parameter here). Hence, the solvability condition on the right side of (3.6.20) is now as follows (with $x=\xi(x) \in \partial \mathscr{D}$ )

$$
\begin{array}{r}
\int_{S^{+}} \bar{U}_{R}(d y ; x)\left[-g(x, y)-b(x, y) u_{0}(x)-\int_{S} \psi(y, d z ; x) F(x, z)\right. \\
\left.. \frac{\partial u_{0}(x)}{\partial x}+\int_{S^{-}} B(x, y, d z) \int_{S} \psi\left(z, d z^{\prime} ; x\right) F\left(x, z^{\prime}\right) \cdot \frac{\partial u_{0}(x)}{\partial x}\right]=0 .
\end{array}
$$

This gives the following boundary conditions for $\iota_{0}(x)$.

(3.6. 21) $\quad \bar{B}(x) \cdot \frac{\partial u_{0}(x)}{\partial x}+\bar{b}(x) u_{0}(x)+\bar{g}(x)=0, \quad x \in \partial \mathscr{D}$,

where

$$
\begin{aligned}
\bar{B}(x)= & \int_{S^{+}} \bar{U}_{R}(d y ; x)\left[\int_{S} \psi(y, d z ; x) F(x, z)\right. \\
& \left.-\int_{S^{-}} B(x, y, d z) \int_{S} \psi\left(z, d z^{\prime} ; x\right) F\left(x, z^{\prime}\right)\right], \quad x \in \partial \mathscr{D},
\end{aligned}
$$

$$
\begin{array}{ll}
\bar{b}(x)=\int_{S^{+}} \bar{U}_{R}(d y ; x) b(x, y), & x \in \partial \mathscr{D}, \\
\bar{g}(x)=\int_{S^{+}} \bar{U}_{R}(d y ; x) g(x, y), & x \in \partial \mathscr{D} .
\end{array}
$$

Equation (3.5.14) and the boundary conditions (3.6.21) determine $\iota_{0}(x)$ uniquely (formally). Note in particular that the term

$$
\bar{a}(x)=\int_{S} \bar{P}(d y ; x) a(x, y)
$$

in (3. 5.15) is strictly negative by (3.6.5) and this is used in the uniqueness. We need however one more condition in order to have (rigorously) existence uniqueness and smoothness of solutions for (3.5.14), (3.6.21). This is the following. 
There is a $\delta_{4}>0$ such that

$$
\hat{n}(x) \cdot \bar{B}(x) \geq \delta_{4} \quad \text { for all } x \in \partial \mathscr{D} .
$$

It is clear that $\hat{n}(x) \cdot \bar{B}(x) \geq 0$ but we must, as we do, exclude tangential reflection at the boundary.

Returning to (3.6.18)-(3.6.20) we see that $u_{1}^{B L}$ is now uniquely determined and has the required properties; in particular (3.6.19) holds exponentially fast. The determination of $u_{2}^{B L}$ with the right properties follows in a similar manner. Now however we must use (2.6.36) which relies on Theorem 2 of Section 2.7. From the solvability condition for $u_{2}^{B L}$ we obtain boundary conditions for $u_{10}$, as usual.

Let us consider briefly the form of the vector field $\bar{B}(x)$ in (3.6.22), in the important special case that

$$
\bar{U}_{R}(A ; x)=\frac{\int_{A} \hat{n}(x) \cdot F(x, y) \bar{P}(d y ; x)}{\int_{S^{+}} \hat{n}(x) \cdot F(x, y) \bar{P}(d y ; x)}, \quad A \subset S^{+} .
$$

This corresponds, of course, to a special choice for the operator $B(x, y$, $A)$. It says, loosely, that reflection at the boundary does not alter the interior equilibrium distribution of velocities (for a moving particle model). If $B(x, y, A)$ satisfies

$$
\begin{aligned}
& \int_{S} \hat{n}(x) \cdot F(x, y) \bar{P}(d y ; x) B(x, y, A) \\
& \quad+\int_{A} \hat{n}(x) \cdot F(x, y) \bar{P}(d y ; x)=0, \text { for all } A \subset S^{-},
\end{aligned}
$$

then indeed (3.6.27) follows. For this we make use of the identity

$$
\begin{aligned}
\int_{S^{-}} \hat{n}(x) & \cdot F(x, y) U(0, y, A ; x) \bar{P}(d y ; x) \\
& +\int_{A} \hat{n}(x) \cdot F(x, y) \bar{P}(d y ; x)=0, \quad \text { for all } A \subset S^{+},
\end{aligned}
$$

where $U(\eta, y, A ; x) \quad(x \in \partial \mathscr{D}$ is a parameter) satisfies (2.6.12) with $z(y) \equiv \hat{n}(x) \cdot F(x, y)$. Recall also that $\bar{U}_{R}$ is the invariant measure of the transition operator $B R$ of (2.6.30).

Using (3.6.27) in (3.6.22) we find that $\bar{B}$ takes the form 
(3. 6. 30) $\quad \bar{B}(x)=\int_{S} \int_{S} \bar{P}(d y ; x) \psi(y, d z ; x) \hat{n}(x) \cdot F(x, y) F(x, z)$.

On comparing (3.6.30) with the differential operator in (3.5.15) we see that $\bar{B}(x)$ is the conormal vector field associated with $\overline{\mathcal{L}}$. Thus, if (3. 6. 27) (or (3.6.28)) holds then (3.6.21) becomes

$$
\frac{\partial u_{0}(x)}{\partial \hat{n}_{\bar{J}}}+\bar{b}(x) u_{0}(x)+\bar{g}(x)=0, \quad x \in \partial \mathscr{D} .
$$

Theorem. Assume that $F, Q, a, B, b, f$ and $g$ depend smoothly on $x \in \overline{\mathscr{D}}$, that $\partial \mathscr{D}$ is smooth. that (3.6.5), (3.6.6), (3.6.7), (3.6.8) and (3.6.26) hold. Assume that $\overline{\mathcal{L}}$ of (3.5.15) is uniformly elliptic in $\bar{D}$. Assume that $Q_{x}$ of (3.5.5) satisfies the Fredholm alternative in the form (2.5.9) and (2.5.10). Then

$$
\sup _{x \in \overline{\mathscr{D}}} \sup _{y \in S}\left|u^{\varepsilon}(x, y)-u_{0}(x)\right|=O(\varepsilon) \text {, }
$$

where $\iota_{0}(x)$ satisfies (3.5.14) and (3.6.21).

Proof. First we observe that our hypotheses imply that $u_{0}(x)$ is smooth along with all other quantities that enter the constructions above.

Define, up to cutoffs,

$$
W^{c}=u^{\varepsilon}-\left(u u_{0}+\varepsilon u_{1}+\varepsilon^{2} u_{2}\right)-\left(\varepsilon u_{1}^{B L}+\varepsilon^{2} u_{2}^{B L}\right) .
$$

By construction we have that'

$$
\mathcal{S}_{-}^{\varepsilon} W^{\S}=O(\varepsilon), \quad x \in \mathscr{D}, y \in S ; x \in \partial \mathscr{D}, y \in S^{-} .
$$

$$
[(B-I)-\varepsilon b] W^{\varepsilon}=O\left(\varepsilon^{3}\right), \quad x \in \partial \mathscr{D}, y \in S^{-} .
$$

We must show that this implies

$$
\left|W^{\varepsilon}\right|=O(\varepsilon),
$$

from which (3.6.32) follows immediately (cf. (3.2.3)).

Let $h_{1}^{\varepsilon}(x, y)$ and $h_{2}^{\varepsilon}(x, y)$ denote the right hand sides of (3.6.34) and (3.6.35) so that $h_{1}^{\varepsilon}=O(\varepsilon)$ and $h_{2}^{\varepsilon}=O\left(\varepsilon^{3}\right)$. From the considerations leading from (2.3.4) to (2.3.5) we conclude that if $\left(X^{s}(t)\right.$, $\left.Y^{\varepsilon}(t)\right)$ is the reflected process generated by

\footnotetext{
tWe employ the obvious operator notation (cf. (3.6.1), (3.6.2)).
} 
144 Alain Bensoussan, Jacques L. Lions and George C. Papanicolaou

(3.6.37) $\left\{\begin{aligned} & \frac{1}{\varepsilon^{2}} Q+\frac{1}{\varepsilon} F \cdot \frac{\partial}{\partial x}, x \in \mathscr{D}, y \in S ; x \in \partial \mathscr{D}, y \in S^{-}, \\ & B-I, x \in \partial \mathscr{D}, y \in S^{+}, \text {(instantaneous reflection) }\end{aligned}\right.$

then, (cf. (2.3.4), (2.3.5), (2.3.6))

(3.6.38)

$$
\begin{aligned}
W^{\varepsilon}(x, y)= & E_{x, y}^{R}\left\{\int_{0}^{\infty} A^{\varepsilon}(s) h_{1}^{\varepsilon}\left(X^{\varepsilon}(s), Y^{\varepsilon}(s)\right) d s\right\} \\
+ & E_{x, y}^{R}\left\{\int_{0}^{\infty} A^{\varepsilon}(s) h_{2}^{\varepsilon}\left(X^{\varepsilon}(s), Y^{\varepsilon}(s-)\right) d N^{\varepsilon}(s)\right\}, \\
A^{\varepsilon}(t)=\exp & \left\{\int_{0}^{t} a\left(X^{\varepsilon}(s), Y^{\varepsilon}(s)\right) d s\right. \\
+ & \left.\int_{0}^{t} \log \left(\frac{1}{1-b\left(x^{\varepsilon}(s), Y^{\varepsilon}(s-)\right)}\right) d N^{\varepsilon}(s)\right\}
\end{aligned}
$$

Here $N^{s}(t)$ is the increasing process that counts returns to the boundary and it is defined as in (2.2.21). Note that by (3.6.5) the first integral on the right side of (3.6.38) is indeed $O(\varepsilon)$. The second integral is majorized by a constant times

$$
\begin{aligned}
y^{\varepsilon}(x, y) & \equiv \varepsilon^{3} E_{x, y}^{R}\left\{\int_{0}^{\infty} e^{-\alpha s} d N^{\varepsilon}(s)\right\}, \quad \alpha>0, \\
& =\lim _{t \uparrow \infty} \varepsilon^{2} E_{x, y}^{R}\left\{\int_{0}^{t} e^{-\alpha s} d\left(\varepsilon N^{\varepsilon}(s)\right)\right\} .
\end{aligned}
$$

The lemma that follows shows that

$$
\widetilde{\nu}^{\varepsilon}(t, x, y) \equiv E_{x, y}^{R}\left\{\int_{0}^{t} e^{-\alpha s} d\left(\varepsilon N^{\varepsilon}(s)\right)\right\}
$$

is bounded independently of $x, y, t$ and $\varepsilon$. This completes the proof of the theorem.

Lemma. Under the hypotheses of the theorem, $\widetilde{\nu}^{s}(t, x, y)$ defined by (3.6.40), is bounded independently of $t \geq 0, x \in \bar{D}, y \in S$, and $0 \leq \varepsilon \leq \varepsilon_{0}$, rith $\alpha>0$ fixed.

Proof of Lemma. Since $\partial \mathscr{D}$ is smooth there exists a function $\phi(x)$ on $\bar{D}$ such that $\phi(x)>0$ for $x \in \mathscr{D}, \phi(x)$ is smooth and bounded, $\phi(x)$ $=0, x \in \partial \mathscr{D}$, and $|\nabla \phi(x)| \geq 1, x \in \partial \mathscr{D}$. For example, for $k>0$ sufficiently large, the solution of 


$$
\lrcorner \phi=-k, \quad x \in \mathscr{D} ; \phi(x)=0, x \in \partial \mathscr{D},
$$

satisfies these conditions.

With $\phi(x)$ so fixed we define $\phi_{1}(x, y)$ as a bounded solution of

(3.6. 41) $\quad Q_{x} \phi_{1}(x, y)+F(x, y) \cdot \frac{\partial \phi(x)}{\partial x}=0, \quad x \in \overline{\mathscr{D}}, y \in S$.

This function $\phi_{1}$ is well defined by the Fredholm alternative for $Q_{x}$ and (3. 6. 6). We define $\phi_{1}^{B L}(\eta, x, y), x \in \partial \mathscr{D}$ as the solution of

$$
\begin{aligned}
& \mathcal{L}_{B L 1} \phi_{1}^{B L}=0, \quad \eta<0, \phi_{1}^{B L} \rightarrow 0 \text { as } \eta \rightarrow-\infty \\
&\left.(B-I) \phi_{1}^{B L}\right|_{\substack{\eta=0 \\
y \in S^{+}}}=\left.\cdots(B-I) \phi_{1}\right|_{\substack{x \in \mathcal{L} \in \mathcal{D} \\
y \in S^{+}}}+\left.\int_{S^{+}} \bar{U}_{R}(B-I) \phi_{1}\right|_{x \in \partial \mathscr{D}} \\
&=-\left.(B-I) \phi_{1}\right|_{\substack{x \in O \mathscr{D} \\
y \in S^{+}}}-\bar{B} \cdot \frac{\partial \phi}{\partial x} \mid
\end{aligned}
$$

We also define

(3. 6. 42) $\phi^{\varepsilon}(x, y)=\phi(x)+\varepsilon \phi_{1}(x, y)+\varepsilon \phi_{1}^{B L}\left(\frac{(x-\xi(x)) \cdot \hat{n}(x)}{\varepsilon}, \xi(x), y\right)$.

It follows that

$$
\begin{aligned}
\left(\frac{1}{\varepsilon^{2}} Q_{x}+\frac{1}{\varepsilon} F \cdot \frac{\partial}{\partial x}\right) \phi^{\varepsilon} & =F \cdot \frac{\partial \phi_{1}}{\partial x}+\varepsilon\left(\mathcal{L}_{B L}^{\varepsilon}-\frac{1}{\varepsilon^{2}} \mathcal{L}_{B L 1}\right) \phi_{1}^{B L} \\
& =\phi^{\varepsilon}=O(1)
\end{aligned}
$$

and

$$
\left.(B-I) \phi^{\varepsilon}\right|_{x \in \partial \mathscr{D}}=-\left.\varepsilon \bar{B} \cdot \frac{\partial \phi}{\partial x}\right|_{x \in \partial \mathscr{D}}
$$

We now use the above in (2.2.20) to obtain the following identity (with $\left.\mathcal{L}^{\varepsilon}=\varepsilon^{-2} Q+\varepsilon^{-1} F \cdot \frac{\partial}{\partial x}\right)$.

(3. 6. 45) $\quad E_{x, y}^{R}\left\{e^{-\alpha t} \phi^{\varepsilon}\left(X^{r \varepsilon}(t), Y^{r \varepsilon}(t)\right)\right\}$

$$
\begin{aligned}
=\phi^{\varepsilon}(x, y) & +\int_{0}^{t} e^{-a s} E_{x, y}^{R}\left\{\left(\mathcal{L}^{\prime}-\alpha\right) \phi^{\varepsilon}\left(X^{\varepsilon}(s), Y^{\varepsilon}(s)\right)\right\} d s \\
& +E_{x, y}^{R}\left\{\int_{0}^{t} e^{-a s}(B-I) \phi^{\varepsilon}\left(X^{\varepsilon}(s), Y^{\varepsilon}(s-)\right) d N^{\varepsilon}(s)\right\} .
\end{aligned}
$$

\footnotetext{
Up to cutoffs as nisual.
} 
Hence, by (3.6.43), (3.6.44) and the boundedness of $\phi$ and its derivatives,

(3.6. 46) $-E_{x, y}^{R}\left\{\int_{0}^{t} e^{-\alpha s} \bar{B}\left(X^{\varepsilon}(s)\right) \cdot \frac{\partial \phi\left(X^{\varepsilon}(s)\right)}{\partial x} d\left(\varepsilon N^{\varepsilon}(s)\right)\right\} \leq c(\alpha)<\infty$, where $c(\alpha)$ is a constant depending only on $\alpha>0$ and not on $x, y, t$ and $\varepsilon \in\left[0, \varepsilon_{0}\right]$.

By construction, $\nabla \phi=\frac{\partial \phi}{\partial x}$ is directed inwards at the boundary and since $|\nabla \phi| \geq 1$ for $x \in \partial \mathscr{D}$ it follows that $\nabla \phi$ is bounded away from tangential directions uniformly in $x \in \partial \mathscr{D}$. Condition (3.6.26) and this observation imply that there is a constant $\delta_{5}>$ such that

$$
-\bar{B}(x) \cdot \frac{\partial \phi(x)}{\partial x} \geq \delta_{5}, \quad x \in \partial \mathscr{D}
$$

Combining (3.6.46) and (3.6.47) we obtain

$$
E_{x, y}^{R}\left\{\int_{0}^{t} e^{-\alpha s} d\left(\varepsilon N^{\varepsilon}(s)\right)\right\} \leq \delta_{5}^{-1} c(\alpha)<\infty,
$$

and the proof of the lemma is complete.

\section{7. Weak Convergence of Reflected Process}

This section is a companion to Section 3.6. We shall remove many of the smoothness requirements but we shall only show weak convergence; the procedure is analogous to the one of Section 3. 3. We begin by formulating the problem anew as follows.

Let $\mathscr{D} \subset \mathbb{R}^{n}$ be a bounded open set such that

$$
\begin{aligned}
& \mathscr{D}=\left\{x \in \boldsymbol{R}^{n}: \phi(x)>0\right\} \\
& \partial \mathscr{D}=\left\{x \in \boldsymbol{R}^{n}: \phi(x)=0\right\},
\end{aligned}
$$

where $\phi(x)$ is defined on $\bar{D}$, has two bounded continuous derivatives and

$$
|\nabla \phi| \geq 1, \quad x \in \partial \mathscr{D},
$$

where $\nabla \phi$ is the gradient and $|x|^{2}=\sum_{i=1}^{n} x_{i}^{2}$.

Let $S$ be a compact metric space and let, for each $x \in \bar{D}, Q_{x}$ denote the generator 


$$
Q_{x} f(y)=q(x, y) \int_{S} \pi(x, y, d z) f(z)-q(x, y) f(y),
$$

defined on continuous functions on $S$. We assume that $q(x, y)$ and $\pi(x, y, A), A$ a Borel subset of $S$, are continuous functions of $x$ and $y$ such that

$$
0<q_{l} \leq q(x, y) \leq q_{u}<\infty
$$

(3. 7. 5) $\quad \pi(x, y, A)$ has density $\tilde{\pi}(x, y, \approx)$ with respect to a reference measure such that $\tilde{\pi}$ is jointly continuous and

$$
0<\tilde{\pi}_{l} \leq \tilde{\pi}(x, y, z) \leq \tilde{\pi}_{u}<\infty .
$$

We assume in addition that $q$ and $\tilde{\pi}$ are continuously differentiable functions of $x$. According to the results of Section 2.5, $Q_{x}$ generates an ergodic (strong) Markov process on $S$ with transition function $P(t, y, A$; $x)$, invariant measure $\bar{P}(A ; x)$ and recurrent potential kernel $\psi(y, A ; x)$. These quantities depend differentiably upon the parameter $x \in \overline{\mathscr{D}}$ as remarked at the end of Section 2.5.

Let $F(x, y)$ be a continuous $n$-vector function on $\bar{D} \times S$ once boundedly differentiable in $x$ and such that

$$
\int_{S} F(x, y) \bar{P}(d y ; x) \equiv 0 .
$$

For each $x \in \partial \mathscr{D}$ let $\hat{n}(x)$ denote the unit outer normal vector. We define $S^{ \pm}$, subsets of $S$, as follows

$$
S^{ \pm}=S^{ \pm}(x)=\{y \in S: \widehat{n}(x) \cdot F(x, y) \geqq 0, \quad x \in \partial \mathscr{D}\} .
$$

Let $B(x, y, A), x \in \partial \mathscr{D}, y \in S^{\tau}, A$ a Borel subset of $S^{-}$, be a probability kernel, continuous in $x$ and $y$ and such that (3.6.7) and (3.6.8) hold.

Under the above hypotheses, and with $\varepsilon>0$ fixed, there exists a unique process $\left(X^{\varepsilon}(t), Y^{\star}(t)\right)$ on ${ }^{\dagger} \bar{G}$ that spends zero time on $\partial G$ and has generator

$$
\begin{aligned}
& \mathcal{S}^{\varepsilon}=\frac{1}{\varepsilon^{2}} Q_{x}+\frac{1}{\varepsilon} F(x, y) \cdot \frac{\partial}{\partial x}, \\
& x \in \mathscr{D}, y \in S, x \in \partial \mathcal{D}, y \in S^{-},
\end{aligned}
$$

1 cf. (2.2.18) and (2.2.19). 


$$
\mathcal{L}^{\varepsilon}=B-I, x \in \partial \mathscr{D}, y \in S^{+} \text {(instantaneous reflection). }
$$

We refer to Section 2.2 for more details. The case of delayed reflection at the boundary (not discussed in Section 2.2) is easily accommodated as follows. The process spends an exponentially distributed length of time at $x \in \partial \mathscr{D}, y \in S^{+}$before switching inward according to $B$. The parameter of the exponential distribution is (properly scaled) $q^{B}(x, y) / \varepsilon$ and so

$$
\mathcal{L}^{\varepsilon}=\frac{q^{B}}{\varepsilon}(B-I), \quad x \in \partial \mathscr{D}, y \in S^{+},
$$

with associated martingale (cf. (2.2.20))

$$
\begin{aligned}
M_{g}^{\varepsilon}(t) & =g\left(t, X^{\varepsilon}(t), Y^{\varepsilon}(t)\right)-\int_{0}^{t} \chi_{G}\left(X^{\varepsilon}(s), Y^{\varepsilon}(s)\right) \\
& \times\left(\frac{\partial}{\partial s}+\mathcal{L}^{\varepsilon}\right) g\left(s, X^{\varepsilon}(s), Y^{\varepsilon}(s)\right) d s-\int_{0}^{t} \chi_{\partial G}\left(X^{\varepsilon}(s), Y^{\varepsilon}(s)\right) \\
& \times\left(\frac{\partial}{\partial s}+\frac{q^{B}}{\varepsilon}(B-I)\right) g\left(s, X^{\varepsilon}(s), Y^{\varepsilon}(s)\right) d s
\end{aligned}
$$

We shall not deal with delayed reflection in what follows but all results (and those of Section 3.6) extend immediately to this case.'

In the case of instantaneous reflection the increasing step process $N^{*}(t)$ at the boundary is defined by $(2.2 .21)$ and the associated martingale by $(2.2 .20)$.

We are interested in showing that $X^{\varepsilon}(t), 0 \leq t \leq T<\infty$, converges weakly as a process on $C([0, T] ; \bar{D})$ to a diffusion Markov process. We shall now introduce this limiting process and some relevant hypotheses about it. Let $f(x)$ be in $C^{2}(\bar{D})$ and define

$$
\begin{aligned}
\overline{\mathcal{L}} f(x)= & \int_{S} \int_{S} \bar{P}(d y ; x) F(x, y) \\
& \cdot \frac{\partial}{\partial x}\left(\phi(y, d z ; x) F(x, z) \cdot \frac{\partial f(x)}{\partial x}\right) .
\end{aligned}
$$

This is a second order elliptic differential operator and we assume that it is (a) uniformly elliptic in $\overline{\mathscr{D}}$ and (b) the diffusion and drift coefficients

\footnotetext{
${ }^{\dagger}$ In fact this case is easier than the one with instantaneous reflection.
} 
are continuous in $\overline{\mathscr{D}}$ (which is compatible with previous assumptions).

Define also, for $x \in \partial \mathscr{D}$, the vector field ${ }^{\dagger}$

$$
\begin{aligned}
(3.7 .10) \bar{B}(x)= & \int_{S^{+}} \bar{U}_{R}(d y ; x)\left[\int_{S^{-}} B(x, y, d z) \int_{S} \psi\left(z, d z^{\prime} ; x\right) F\left(x, z^{\prime}\right)\right. \\
& \left.-\int_{S} \psi(y, d z ; x) F(x, z)\right] .
\end{aligned}
$$

Here, $\bar{U}_{R}(A ; x), A \subset S^{\dagger}$, is the invariant measure of the operator $B R$ defined by (2.6.29) and $x \in \partial \mathscr{D}$ is a parameter. We assume that there is a $\delta_{4}>0$ such that

$$
\nabla \phi(x) \cdot \bar{B}(x) \geq \delta_{4} . \quad x \in \partial \mathscr{D} .
$$

In addition we assume that $\bar{B}(x)$ is uniformly Lipschitz continuous on $\partial \mathscr{D}$.

Under the abore hypotheses the submartingale problem for $\{\overline{\mathcal{L}}, \bar{B}\}$ has a unique solution according to the theory of Stroock and Varadhan [6]. This means that there is a unique process (measure) $X(t)$ on $C([0, T] ; \overline{\mathscr{D}})$ such that $\mathrm{X}(0)=x$ with probability one and for each smooth $f(x)$ on $\overline{\mathscr{D}}$ such that $\bar{B}(x) \cdot(\partial f(x) / \partial x) \geq 0, x \in \partial \mathscr{D}$,

$$
f(X(t))-\int_{0}^{t} \overline{\mathcal{L}} f(X(s)) d s
$$

is a submartingale. Moreover this process is Feller continuous and hence a strong Markov process.

Theorem. Under the above hypotheses, $X^{\varepsilon}(t)$ converges weakly (as a process on $C([0, T] ; \bar{D})$ ) as $\varepsilon \rightarrow 0,0 \leq t \leq T<\infty$, to the diffusion Markov process $X(t)$ on $\overline{\mathscr{D}}$ associated with $\{\overline{\mathcal{L}}, \bar{B}\}$ of (3.7.9) and (3. 7. 10).

Proof. The proof goes, as usual in two steps. Step 1 consists in showing that for $0 \leq \varepsilon \leq 1$, say, the processes $X^{\varepsilon}(\cdot)$ are compact and step 2 consists in identifying the limits with the process associated (uniquely) with $\{\overline{\mathcal{L}}, \bar{B}\}$. We begin with the compactness.

Since the processes $X^{\varepsilon}(\cdot)$ are continuous and the limit process is

+ We change the sign in (3.6.22) to conform with the notation of Stroock and Varadhan [6]. 
continuous it suffices to show compactness in $D([0, T] ; \overline{\mathscr{D}})$ ( $\overline{\mathscr{D}}$ is also bounded). Hence [20], it suffices to show that

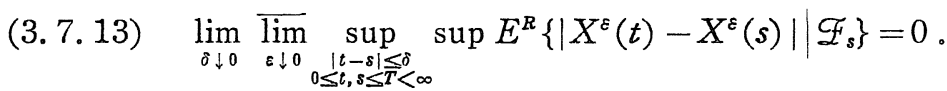

Here, the sup next to the expectation is over the past up to time $s$ i.e., sup over all $x=X^{\varepsilon}(s) \in \bar{D}, y=Y^{\mathfrak{c}}(s) \in S$.

The following considerations are relevant for both the compactness and the identification. Let $f(x)$ be a smooth function and let $f_{1}(x, y)$ be defined by

$$
f_{1}(x, y)=\int_{s} \psi(y, d z ; x) F(x, z) \cdot \frac{\partial f(x)}{\partial x}
$$

In some fixed neighborhood of $\partial \mathscr{D}$ we define the boundary layer function $f_{1}^{B L}(\eta, \xi(x), y) \quad$ (cf. Section 3.4) as the solution of the problem (cf. (3. 4.10))

$$
\mathcal{L}_{B L 1} f_{1}^{B L}=0, \quad \eta<0,
$$

$$
f_{1}^{B L}(\eta, \xi(x), y) \rightarrow 0 \text { as } \eta \rightarrow-\infty \text { (uniformly in } y \text { ), }
$$

$$
\left.(B-I) f_{1}^{B L}\right|_{\substack{\eta=0 \\ y \in S^{+}}}=-\left.(B-I) f_{1}\right|_{\substack{x \in \partial \mathscr{D} \\ y \in S^{+}}}+\left.\bar{B} \cdot \frac{\partial f}{\partial x}\right|_{\substack{x \in \partial \mathscr{D} \\ .0}} .
$$

This problem has a unique solution, differentiable in $(\eta, \xi)$, because the right hand side of (3.7.17) satisfies the appropriate solvability condition by definition of $\bar{B}$ in (3.7.10) (cf. Section 2.6). Now define

$$
\begin{aligned}
f^{\varepsilon}(x, y)= & f(x)+\varepsilon f_{1}(x, y) \\
& +\varepsilon f_{1}^{B L}\left(\frac{(x-\xi(x)) \cdot \hat{n}(x)}{\varepsilon}, \xi(x), y\right),
\end{aligned}
$$

where $f_{1}^{B L}$ is defined (in a differentiable manner) as zero outside a fixed neighborhood of $\partial \mathscr{D}$ (cutoff).

From the construction above it follows that for all $x \in \mathscr{D}, y \in S$ and $x \in \partial \mathscr{D}, y \in S^{-}$

$$
\text { (3.7.19) } \begin{aligned}
\mathcal{L}^{\varepsilon} f^{\varepsilon}(x, y) & =F(x, y) \cdot \frac{\partial f_{1}(x, y)}{\partial x}+\varepsilon\left(\mathcal{L}_{B L}^{\varepsilon}-\frac{1}{\varepsilon^{2}} \mathcal{L}_{B L 1}\right) f_{1}^{B L} \\
& =F(x, y) \cdot \frac{\partial f_{1}(x, y)}{\partial x}+g_{1}^{\varepsilon}(x, y),
\end{aligned}
$$


where $g_{1}^{\varepsilon}(x, y)$ is bounded independently of $\varepsilon$ and is zero outside a fixed neighborhood $^{\dagger}$ of $\partial \mathscr{D}$. The same construction of $f^{\varepsilon}$ yields that for $x \in \partial \mathscr{D}, y \in S^{+}$

$$
(B-I) f^{\varepsilon}(x, y)=\varepsilon \bar{B}(x) \cdot \frac{\partial f(x)}{\partial x}
$$

It follows that (cf. (2.2.20))

$$
\begin{aligned}
M_{f^{\varepsilon}}(t)=f^{\varepsilon} & \left(X^{\varepsilon}(t), Y^{\varepsilon}(t)\right)-f(x, y) \\
& -\int_{0}^{t}\left(F \cdot \frac{\partial f_{1}}{\partial x}+g^{\varepsilon}\right)\left(X^{\varepsilon}(s), Y^{\varepsilon}(s)\right) d s \\
& -\int_{0}^{t}\left(\bar{B} \cdot \frac{\partial f}{\partial x}\right)\left(X^{\varepsilon}(s)\right) d\left(\varepsilon N^{\varepsilon}(s)\right)
\end{aligned}
$$

is a zero mean, bounded martingale. As in (3.3.9), (3.3.10), the increasing process associated with this martingale is given (after a short computation) by

$$
\begin{aligned}
\left\langle M_{f^{\varepsilon}}(t)\right\rangle= & \int_{0}^{t}\left[\mathcal{L}^{\varepsilon}\left(f^{\varepsilon}\right)^{2}-2 f^{\varepsilon} \mathcal{L}^{\varepsilon} f^{\varepsilon}\right]\left(X^{\varepsilon}(s), Y^{\varepsilon}(s)\right) d s \\
& +\int_{0}^{t}\left[(B-I)\left(f^{\varepsilon}\right)^{2}-2 f^{\varepsilon}(B-I) f^{\varepsilon}\right. \\
& \left.-\left((B-I) f^{\varepsilon}\right)^{2}\right]\left(X^{\varepsilon}(s), Y^{\varepsilon}(s-) d N^{\varepsilon}(s)\right. \\
= & \int_{0}^{t}\left[Q f_{1}^{2}-2 f_{1} Q f_{1}+g_{2}^{\varepsilon}\right]\left(X^{\varepsilon}(s), Y^{\varepsilon}(s)\right) d s \\
& +\varepsilon \int_{0}^{t}\left[(B-I)\left(f_{1}+f_{1}^{B L}\right)^{2}-2\left(f_{1}+f_{1}^{B L}\right)(B-I)\right. \\
& \left.\times\left(f_{1}+f_{1}^{B L}\right)-\left(\bar{B} \cdot \frac{\partial f}{\partial x}\right)^{2}\right]\left(X^{\varepsilon}(s), Y^{\varepsilon}(s-)\right) \\
& \times d\left(\varepsilon N^{\varepsilon}(s)\right),
\end{aligned}
$$

where $g_{2}^{\varepsilon}$ is similar to $g_{1}^{\varepsilon}$ above and the integrand in the second integral is bounded.

Now we are in a position to prove (3.7.13). We use (3.7.21) with $f(x)=x_{i}, i=1,2, \cdots, n$ successively or, with vector notation $f(x)=x$, so

${ }^{\dagger} g_{1}^{\varepsilon}(x, y) \rightarrow 0$ exponentially fast as $\varepsilon \downarrow 0$ for each $x$ in this neighborhood and not on $\partial \mathscr{D}$ in view of (3.7.16). Same for $g_{2}^{\varepsilon}$ in (3.7.22). 
that $^{\dagger}$

$$
\begin{aligned}
\mid X^{\varepsilon}(t) & -\left.X^{\varepsilon}(s)\right|^{2} \leq c_{1} \varepsilon^{2}+c_{2}(t-s)^{2} \\
& +4\left|\int_{s}^{t} \bar{B}\left(x^{\varepsilon}(s)\right) d\left(\varepsilon N^{\varepsilon}(s)\right)\right|^{2}+4\left|M^{\varepsilon}(t)-M^{\varepsilon}(s)\right|^{2} .
\end{aligned}
$$

Hence

$$
\begin{gathered}
E^{R}\left\{\left|X^{\varepsilon}(t)-X^{\varepsilon}(s)\right| \mid \mathscr{F}_{s}\right\} \leq\left(E^{R}\left\{\left|X^{\varepsilon}(t)-X(s)\right|^{2} \mid \mathscr{F}_{s}\right\}\right)^{1 / 2} \\
\leq c_{1} \varepsilon^{2}+c_{2}(t-s)^{2}+4 E^{R}\left\{\left|\int_{s}^{t} \bar{B}\left(X^{\varepsilon}(s)\right) d\left(\varepsilon N^{\varepsilon}(s)\right)\right|^{2} \mid \mathscr{F}_{s}\right\} \\
+4 E^{R}\left\{\left|\left\langle M^{\varepsilon}(t)\right\rangle-\left\langle M^{\varepsilon}(s)\right\rangle\right|^{2} \mid \mathscr{F}_{s}\right\}
\end{gathered}
$$

Comparing (3.7.23) with (3.7.13) and using the fact that $\bar{B}$ is bounded and the integrands on the right side of (3.7.22) are bounded, it follows that (3.7.13) will be proved if we show that

$$
E^{R}\left\{\left(\varepsilon N^{\varepsilon}(t)-\varepsilon N^{c}(s)\right)^{2} \mid \mathscr{E}_{s}\right\} \leq c_{3} \varepsilon^{2}+c_{4}(t-s) .
$$

Here $c_{3}$ and $c_{4}$ are constants independent of $\varepsilon, t$ and $s(0 \leq t, s \leq T<\infty)$.

To prove (3.6.24) we proceed as follows [6]. Let $\phi(x)$ be the support function of $\mathscr{D}$ as defined by (3.7.1) and take $f(x) \equiv \phi(x)$ in (3. 7.18). We do this in order to exploit the hypothesis (3.7.11) which says that the limit process is reflected in a strictly interior direction. From (3.7.21), with* $f^{\varepsilon}=\phi^{\varepsilon}$, and (3.7.11) it follows that

$$
\begin{aligned}
\delta_{4} E^{R}\{ & \left.\left(\varepsilon N^{\varepsilon}(t)-\varepsilon N^{\varepsilon}(s)\right)^{2} \mid \mathscr{F}_{s}\right\} \\
\leq & E^{R}\left\{\left(\int_{s}^{t} \bar{B} \cdot \frac{\partial \phi}{\partial x}\left(X^{\varepsilon}(s)\right) d\left(\varepsilon N^{\varepsilon}(s)\right)\right)^{2} \mid \mathscr{I}_{s}\right\} \\
\leq & c(t-s)^{2}+E^{R}\left\{\left(\phi^{\varepsilon}(t)-\phi^{\varepsilon}(s)\right)^{2} \mid \mathscr{F}_{s}\right\} \\
& +E^{R}\left\{\left\langle M_{\phi^{\varepsilon}}(t)\right\rangle-\left\langle M_{\phi \varepsilon}(s)\right\rangle \mid \mathscr{F}_{s}\right\} \\
\leq & c^{\prime}(t-s)+E^{R}\left\{\left(\phi^{\varepsilon}(t)\right)^{2}-\left(\phi^{\varepsilon}(s)\right)^{2} \mid \mathscr{L}_{s}\right\} \\
& \quad-2 \phi^{\varepsilon}(s) E^{R}\left\{\phi^{\varepsilon}(t)-\phi^{\varepsilon}(s) \mid \mathscr{F}_{s}\right\} \\
& +\varepsilon\left(E^{R}\left\{\left(\varepsilon N^{\varepsilon}(t)-\varepsilon N^{\varepsilon}(s)\right)^{2} \mid \mathscr{F}_{s}\right\}\right)^{1 / 2},
\end{aligned}
$$

where $c$ and $c^{\prime}$ are constants. Away from a neighborhood of the bound-

${ }^{1} M^{c}(t)$ is the vector valued martingale corresponding to $f(x)=x$.

* $\phi^{\varepsilon}=\phi_{1}+\varepsilon \phi_{1}+\varepsilon \phi_{1}^{B L}$; also we write $\phi^{\varepsilon}(t)=\phi^{\varepsilon}\left(X^{\varepsilon}(t), Y^{\varepsilon}(t)\right)$. 
ary of order $\varepsilon, \phi^{\varepsilon}$ is $\geq 0$ and near the boundary is $O(\varepsilon)$. Using this observation, (3.7.21) and (3.7.11) we estimate the third term on the right side of (3.7.25) and this leads to

$$
\begin{aligned}
& E^{R}\left\{\left(\varepsilon N^{\varepsilon}(t)-\varepsilon \Lambda^{\varepsilon \varepsilon}(s)\right)^{2} \mid \mathscr{F}_{s}\right\} \\
& \leq c^{\prime \prime}(t-s)+\varepsilon \tilde{c}\left(E^{R}\left\{\left(\varepsilon N^{\varepsilon}(t)-\varepsilon N^{c}(s)\right)^{2} \mid \mathscr{F}_{s}\right\}\right)^{12} \\
& \quad+E^{R}\left\{\left(\phi^{\varepsilon}(t)\right)^{2}-\left(\phi^{\varepsilon}(s)\right)^{2} \mid \mathscr{F}_{s}\right\} .
\end{aligned}
$$

The last term on the right side of (3.7.26) is estimated by using (3. 7. 21) with $f^{z}=\left(\phi^{\varepsilon}\right)^{2}$. It can be verified easily that $\mathcal{L}^{\varepsilon}\left(\phi^{\varepsilon}\right)^{2}$ is bounded independently of $\varepsilon$ and since $\phi=0$ on $\partial \mathscr{D} \phi B \cdot \nabla \phi$ drops out. Therefore, for some constant $c$ we have

$$
\begin{aligned}
E^{R}\{( & \left.\left.\varepsilon N^{s}(t)-\varepsilon N^{\varepsilon}(s)\right)^{2} \mid \mathscr{I}_{s}\right\} \\
& \leq c(t-s)+\varepsilon c\left(E^{R}\left\{\left(\varepsilon N^{s}(t)-\varepsilon N^{s}(s)\right)^{2} \mid \mathscr{F}_{s}\right\}\right) ! 2
\end{aligned}
$$

and this leads to (3.7.24). This completes the prool of (3.7.13) and hence compactness of $X^{\varepsilon}(\cdot)$ in $D$.

We pass now to the second step in the proof which consists of showing that for any smooth $f(x)$ with $\bar{B}(x) \cdot \frac{\partial f(x)}{\partial x} \geq 0$ for $x \in \partial \mathscr{D}$ the quantity

$$
f(X(t))-\int_{0}^{t} \overline{\mathcal{L}} f(X(s)) d s
$$

is a submartingale with respect to any limit measure of the $X^{*}(\cdot)$ process. Our main tool is again the martingale (3. 7.21) with $f(x)$ smooth on $\bar{D}$ and such that $\bar{B}(x) \cdot \frac{\partial f(x)}{\partial x} \geq 0$ for $x \in \partial \mathscr{D}$.

We may rewrite (3.7.21) as follows:

$$
\begin{gathered}
f\left(X^{\varepsilon}(t)\right)-f\left(X^{\varepsilon}(s)\right)-\int_{s}^{t} \overline{\mathcal{L}} f\left(X^{\varepsilon}(s)\right) d s \\
\quad-\int_{s}^{t} \bar{B} \cdot \frac{\partial f}{\partial x}\left(X^{\varepsilon}(s)\right) d\left(\varepsilon N^{\varepsilon}(s)\right) \\
=\varepsilon\left[-f_{1}\left(X^{\varepsilon}(t), Y^{\varepsilon}(t)\right)-f_{1}^{B L}\left(X^{\varepsilon}(t), Y^{\varepsilon}(t)\right)\right. \\
\left.+f_{1}\left(Y^{\varepsilon}(s), Y^{\varepsilon}(s)\right)+f_{1}^{B L}\left(X^{\varepsilon}(s), Y^{\varepsilon}(s)\right)\right] \\
+\int_{s}^{t}\left(F \cdot \frac{\partial f_{1}}{\partial x}-\overline{\mathcal{L}} f^{\prime}\right)\left(X^{\varepsilon}(s), Y^{\varepsilon}(s)\right) d s
\end{gathered}
$$




$$
+\int_{s}^{t} g_{1}^{\varepsilon}\left(X^{\varepsilon}(s), Y^{\varepsilon}(s)\right) d s+M_{f^{\varepsilon}}(t)-M_{f^{\varepsilon}}(s) .
$$

Here we write $f_{1}^{B L}(x, y)$ for simplicity even though $f_{1}^{B L}$ is defined in terms of boundary layer coordinates. Define

$$
A_{f}(x, y)=F(x, y) \cdot \frac{\partial f_{1}(x, y)}{\partial x}-\overline{\mathcal{L}} f(x) .
$$

In a manner entirely analogous to the one of Section 3.3 we can use a preliminary smoothing of $A_{f}$ as a function of $x$ and (3.7.24) to show that

$$
\lim _{\varepsilon \downarrow 0} E^{R}\left\{\int_{s}^{t} A_{f}\left(X^{\varepsilon}(s), Y^{\varepsilon}(s)\right) d s \mid \mathscr{F}_{s}\right\}=0,
$$

uniformly in $x=X^{\varepsilon}(s) \in \bar{D}, y=Y^{\varepsilon}(s) \in S$.

With (3.7.30) on hand the desired characterization of the limiting process will follow immediately from (3.7.28) if we can show that

$$
\lim _{\varepsilon \downarrow 0} E^{R}\left\{\int_{s}^{t} g_{1}^{\varepsilon}\left(X^{\varepsilon}(s), Y^{\varepsilon}(s)\right) d s \mid \mathscr{F}_{s}\right\}=0 .
$$

Recall that $g_{1}^{\varepsilon}(x, y)$ is a function that has support in a fixed neighborhood of the boundary, it is bounded independently of $\varepsilon$ and goes to zero uniformly in $y$ as $\varepsilon \rightarrow 0$ for $x$ in any closed subset not containing boundary points.

Let $\mathscr{D}_{\delta}=\{x \in \overline{\mathscr{D}}:|x-\partial \mathscr{D}|<\delta\}$ and $\mathscr{D}_{\delta}^{c}=\overline{\mathscr{D}}-\mathscr{D}_{\delta}$. Then

$$
\begin{aligned}
& \left|E^{R}\left\{\int_{s}^{t} g_{1}^{\varepsilon}\left(X^{\varepsilon}(s), Y^{\varepsilon}(s)\right) d s \mid \mathscr{F}_{s}\right\}\right| \\
& \leq\left|E^{R}\left\{\int_{s}^{t} \chi_{\mathscr{D}_{\delta}^{\varepsilon}}\left(X^{\varepsilon}(s)\right) g_{1}^{\varepsilon}\left(X^{\varepsilon}(s), Y^{\varepsilon}(s)\right) d s \mid \mathscr{F}_{s}\right\}\right| \\
& \quad+C E^{R}\left\{\int_{s}^{t} \chi_{\mathscr{D}_{\delta}}\left(X^{\varepsilon}(s)\right) d s \mid \mathscr{F}_{s}\right\},
\end{aligned}
$$

where $C$ is a constant. The first term on the right side of (3.7.32) goes to zero as $\varepsilon \rightarrow 0$. We must therefore show that

$$
\lim _{\hat{\delta} \downarrow 0} \varlimsup_{\varepsilon \downarrow 0} \sup _{x, y} E_{x, y}^{R}\left\{\int_{0}^{t} \chi_{\mathscr{S}_{\delta}}\left(X^{\varepsilon}(s)\right) d s\right\}=0,
$$

where we have set $s=0$ and $t=T<\infty$ without loss in generality.

To prove (3.7.33) we adapt again a device of Stroock and Varadhan 
[6]. Let $(\delta>0$ is fixed $)$

$$
\eta_{\delta}(r)= \begin{cases}\frac{1}{\delta}(\delta-r)^{3}, & 0 \leq r \leq \delta \\ 0 & r \geq 0,\end{cases}
$$

$$
\hat{\phi}_{\delta}(x)=\eta_{\hat{o}}(\phi(x))=\eta_{\delta} \circ \phi(x) \text {, }
$$

where $\phi(x)$ is the support function of $\mathscr{D}$. With $\widehat{\phi}_{\delta}(x)$ as the function $f(x)$ we construct $\hat{\phi}_{\hat{\delta}, 1}(x, y)$ according to (3.7.14) and set

$$
\widehat{\phi}_{\hat{\delta}}^{\varepsilon}(x, y)=\widehat{\phi}_{\delta}(x)+\varepsilon \widehat{\phi}_{\delta, 1}(x, y),
$$

$$
\widehat{\phi}_{\delta, 1}(x, y)=\eta_{\delta}^{\prime}(\phi(x)) \int \psi(x, y, d \approx) F(x, \approx) \cdot \frac{\partial \phi(x)}{\partial x} .
$$

Then,

(3.7.37) $\mathcal{L}^{\varepsilon} \hat{\phi}_{\delta}^{\varepsilon}(x, y)=\overline{\mathcal{L}} \hat{\phi}_{\delta}(x)+A_{\hat{\phi}_{\delta}}(x, y)+O(\varepsilon), \quad x \in \mathscr{D}, y \in S$, using the notation (3.7.9) and (3.7.29), and

$$
(B-I) \widehat{\phi}_{\hat{\delta}}^{\varepsilon}(x, y)=\varepsilon(B-I) \widehat{\phi}_{\delta, 1}(x, y), \quad x \in \partial \mathscr{D}, y \in S^{+} .
$$

It is convenient to write the operator $\overline{\mathcal{L}}$ of (3.7.9) in the usual form

$$
\overline{\mathcal{L}}=\frac{1}{2} \sum_{i, j=1}^{n} \bar{a}_{i j}(x) \frac{\partial^{2}}{\partial x_{i} \partial x_{j}}+\sum_{j=1}^{n} \bar{b}_{j}(x) \frac{\partial}{\partial x_{j}} .
$$

It follows from (3.7.35) that

$$
\text { (3. 7. 39) } \overline{\mathcal{L}} \hat{\phi}_{\delta}(x)=\eta_{\delta}^{\prime \prime}(\phi(x)) \frac{1}{2} \sum_{i, j=1}^{n} \bar{a}_{i j}(x) \frac{\partial \phi}{\partial x_{i}} \frac{\partial \phi}{\partial x_{j}}+\eta_{\delta}^{\prime}(\phi(x)) \overline{\mathcal{L}} \phi(x) \text {. }
$$

Combining (3.7.39), (3.7.34) and (3.7.35) in the martingale (2.2.20) we obtain

$$
\begin{aligned}
& E_{x, y}^{R}\left\{\int_{0}^{t}\left(\eta_{\delta}^{\prime \prime} \circ \phi \sum_{i, j=1}^{n} \bar{a}_{i j} \frac{\partial \phi}{\partial x_{i}} \frac{\partial \phi}{\partial x_{j}}\right)\left(X^{\varepsilon}(s)\right) d s\right\} \\
& \leq c_{1} \delta^{2}+c_{2} \varepsilon \delta+\delta c_{3} T+2\left|E_{x, y}^{R}\left\{\int_{0}^{T} A_{\hat{\phi}_{8}}\left(X^{\varepsilon}(s), Y^{\varepsilon}(s)\right) d s\right\}\right| \\
& \quad+c_{4} \delta E_{x, y}^{R}\left\{\varepsilon N^{\varepsilon}(T)\right\}
\end{aligned}
$$

where $c_{1}, c_{2}, c_{3}$ and $c_{4}$ are constants. Using (3.7.24) and (3.7.30) yields the results 
(3. 7. 40) $\lim _{\hat{\sigma} \downarrow 0} \varlimsup_{\varepsilon \downarrow 0} \sup _{x, y} E_{x, y}^{R}\left\{\int_{0}^{T} \eta_{\hat{o}}^{\prime} \circ \phi \sum_{i, j=1}^{n} \bar{a}_{i j} \frac{\partial \phi}{\partial x_{i}} \frac{\partial \phi}{\partial x_{i}}\left(X^{\varepsilon}(s)\right) d s\right\}=0$.

We observe now that (i) the coefficients $\left(\bar{a}_{i j}\right)$ are uniformly elliptic in $\bar{D}$, (ii) $|\nabla \phi| \geq 1$ for $x \in \partial \mathscr{D}$ and $\nabla \phi$ is continuous in $\overline{\mathscr{D}}$ and (iii) $\eta_{0}^{\prime \prime}(\phi(x)) \geq \frac{1}{2}$, say, for $x \in \mathscr{D}_{\delta^{\prime}}$ with $\delta^{\prime}$ going to zero as $\delta$ goes to zero. Thus, for some $r>0$,

$$
\begin{aligned}
\gamma E_{x, y}^{R}\left\{\int_{0}^{T} \chi_{\mathscr{D}_{\delta^{\prime}}}\left(X^{\varepsilon}(s)\right) d s\right\} \\
\quad \leq E_{x, y}^{R}\left\{\int_{0}^{T} \eta_{\delta}^{\prime \prime} \circ \phi \sum_{i, j=1}^{n} \bar{a}_{i j} \frac{\partial \phi}{\partial x_{i}} \frac{\partial \phi}{\partial x_{j}}\left(X^{\varepsilon}(s)\right) d s\right\}
\end{aligned}
$$

and hence (3. 7.40) proves the desired result (3.7.33). The proof of the theorem is complete.

\section{References}

[1] Papanicolaou, G., Asymptotic analysis of transport processes, Bulletin of the A. M. S., 81 (1975), 330-392.

[2] Babuska, I., Homogenization and its applications, Univ. of Maryland Technical Note BN-821, July 1975, and many other reports from the University of Maryland.

[3] Bensoussan, A., Lions, J. L. and Papanicolaou, G. C., Asymptotic Analysis for Periodic Structures, North Holland, 1978. See also C. R. Acad. Sc. Paris, 281 (A) (July 1975), 89-94 and 317-322, and also 282 (A) (January 1976), 143-147 and 1277-1282.

[4] Larsen, E. and Keller, J. B., Asymptotic solution of neutron transport problems for small mean free paths, J. Math. Phys., 15 (1974), 75-81.

[5] Larsen, E., Solution of the steady, one-speed neutron transport equation for small mean free path., J. Math. Phys., 15 (1974), 299-305.

- and D'Arruda, J., Asymptotic theory of the linear transport equation for small mean free paths I., Phys. Rev. A, 13 (1976), 1933, Part II to appear in SIAM J. Appl. Math.

- Neutron transport and diffusion in inhomogenous media, I., J. Math. Phys., 16 (1975), 1421-1427, Part II to appear in Nuclear Science and Engineering.

[6] Stroock, D. and Varadhan, S. R. S., Diffusion processes with boundary condition, Comm. Pure Appl. Math., 24 (1971), 147-225.

[7] Kurtz, T. G., Semigroups of conditioned shifts and approximation of Markov processes, Ann. Probability, 3 (1975), 618-642.

[8] Williams, M., Dissertation, N. Y. U. February 1976.

[9] Chandrasekhar, S., Radiative transfer, Dover, 1960.

[10] Weinberg, A. and Wigner, E., The Physical theory of neutron chain reactors, University of Chicago Press, 1958.

[11] Case, K. M. and Zweifel, P. F., Linear transport theory, Addison-Wesley, Reading, Mass., 1967.

[12] Williams, M. M. R., Mathematical methods in particle transport theory, Wiley, New York, 1971.

[13] Davison, B. and Sykes, J. B., Neutron transport theory, Clarendon Press, Oxford England, 1957. 
[14] Kogan, M. N., Rarefied gas dynamics, P'enum Press, New York, 1969.

[15] Grad, H., Singular and nonuniform limits of colutions of the Boltzmann equation, SIAM-AMS Proc., 1, Amer. Math. Soc., Providence, R. I., (1969), 269-308.

[16] Chapman, S. and Cowling, T. G., The mathematical theory of nonuniform gases, Cambridge Univ. Press, Cambridge England, 1952.

[17] Doob, J. L., Stochastic processes, Wiley, New York, 1953.

[18] Stroock, D. and Varadhan, S. R. S., Diffusion processes with continuous coefficients I and II, Comm. Pure Appl. Math. 22 (1969), 345-400 and 479-530.

[19] Billingsley, P., Convergence of probability measures, Wiley, New York, 1968.

[20] Gihman, I. I. and Skorohod, A. V., The theory of stochastic processes I, Springer, New York, 1974.

[21] Papanicolaou, G. C., Boundary behavior of branching transport processes, Stochastic Analysis, edited by A. Friedman and M. Pinsky, Academic Press, New York, (1978), 215-237. 
